\title{
MODEL-THEORETIC APPLICATIONS OF COFINALITY SPECTRUM PROBLEMS
}

\author{
M. MALLIARIS AND S. SHELAH
}

\begin{abstract}
We apply the recently developed technology of cofinality spectrum problems to prove a range of theorems in model theory. First, we prove that any model of Peano arithmetic is $\lambda$-saturated iff it has cofinality $\geq \lambda$ and the underlying order has no $(\kappa, \kappa)$-gaps for regular $\kappa<\lambda$. Second, assuming instances of $\mathrm{GCH}$, we prove that $\mathrm{SOP}_{2}$ characterizes maximality in the interpretability order $\triangleleft^{*}$, settling a prior conjecture and proving that $\mathrm{SOP}_{2}$ is a real dividing line. Third, we establish the beginnings of a structure theory for $\mathrm{NSOP}_{2}$, proving for instance that $\mathrm{NSOP}_{2}$ can be characterized in terms of few inconsistent higher formulas. In the course of the paper, we show that $\mathfrak{p}_{\mathbf{s}}=\mathfrak{t}_{\mathbf{s}}$ in any weak cofinality spectrum problem closed under exponentiation (naturally defined). We also prove that the local versions of these cardinals need not coincide, even in cofinality spectrum problems arising from Peano arithmetic.
\end{abstract}

In a recent paper [8] we connected and solved two a priori unrelated open questions: the question from model theory of whether $\mathrm{SOP}_{2}$ is maximal in Keisler's order, and the question from set theory/general topology of whether $\mathfrak{p}=\mathfrak{t}$. This work was described in the research announcement [9] and the commentary [11. In order to prove these theorems, we introduced a general framework called cofinality spectrum problems, reviewed in \$1 below.

In the present paper, we develop and apply cofinality spectrum problems to a range of problems in model theory, primarily on Peano arithmetic and around the strong tree property $\mathrm{SOP}_{2}$, also called the 2 -strong order property. We prove the following theorems:

Theorem (Theorem 4.7). Let $N$ be a model of Peano arithmetic, or just bounded $P A$, and $\lambda$ an uncountable cardinal. If the reduct of $N$ to the language of order has cofinality $>\kappa$ and no $(\kappa, \kappa)$-cuts for all $\kappa<\lambda$, then $N$ is $\lambda$-saturated.

On the earlier history, which involves [13] and [16] VI.2-3, see \$4 We also address a question about balanced pairs of models of PA. The proof of Theorem 4.7 relies on the answer to a question arising from [8], of intrinsic interest:

1991 Mathematics Subject Classification. 03C20, 03C45, 03E17.

Key words and phrases. Unstable model theory, cardinal invariants of the continuum, $\mathfrak{p}$ and $\mathfrak{t}$, cofinalities of cuts, interpretability order $\triangleleft^{*}$, Peano arithmetic, $\mathrm{SOP}_{2}$.

Thanks: Malliaris was partially supported by NSF grants 1300634 and by a Sloan fellowship. Shelah was partially supported by the Israel Science Foundation grants 710/07 and 1053/11 and European Research Council grant 338821. This is manuscript 1051 in Shelah's list of publications. 
Theorem (Theorem 2.10. Let $\mathbf{s}$ be a cofinality spectrum problem which is closed under exponentiation. Then $\mathfrak{p}_{\mathbf{s}}=\mathfrak{t}_{\mathbf{s}}$.

As explained in $\$ 1$. Theorem 2.10 complements a main theorem of 8 , which showed that $\mathfrak{t}_{\mathbf{s}} \leq \mathfrak{p}_{\mathbf{s}}$ for any cofinality spectrum problem $\mathbf{s}$. As a consequence, we are able to characterize $\mathfrak{t}_{\mathbf{s}}$ in terms of the first symmetric cut. However, as we show in $\$ 5$, the local versions of these cardinals, $\mathfrak{p}_{\mathbf{s}, \mathbf{a}}, \mathfrak{t}_{\mathbf{s}, \mathbf{a}}$ need not coincide unless the underlying model is saturated.

We then turn to the strong tree property $S_{\mathrm{OP}}$. A major result of [8] was that $S O P_{2}$ suffices for being maximal in Keisler's order $\triangleleft$. It was not proved to be a necessary condition, but we conjectured there that $\mathrm{SOP}_{2}$ characterizes maximality in Keisler's order. The difficulty in addressing this question may be in building ultrafilters. However, in the present paper, for a related open problem, we give a complete answer:

Theorem (Theorem 6.14, under relevant instances of GCH). $T$ is $\triangleleft^{*}$-maximal if and only if it has $\mathrm{SOP}_{2}$.

The ordering $\triangleleft^{*}$ refines Keisler's order, but is defined not in terms of ultrapowers but rather in terms of interpretability. Theorem 6.14 answers a very interesting question going back to Džamonja and Shelah [1] and Shelah and Usvyatsov [24. We inherit the assumption of a case of GCH from 24, where one direction of the theorem was proved, building on work of 1 . The direction proved here is in ZFC.

Theorem 6.14 gives decisive evidence for $\mathrm{SOP}_{2}$ being a dividing line, by giving the equivalence of a natural inside/syntactic property and an outside property. However, this was done without developing a structure theory. So in $\$ 8$, we develop the beginnings of a structure theory for $\mathrm{NSOP}_{2}$. We define a notion of 'higher formulas' using ultrafilters and prove, for example, that:

Theorem (Theorem 8.21). T is $\mathrm{NSOP}_{2}$ iff for all infinite $A$, the number of pairwise 1-contradictory higher $\varphi$-formulas over $A$ is $\leq|A|$.

Section 8 contains several other results, notably the "symmetry lemma" states that $N S O P_{3}$ can be characterized in terms of symmetry of inconsistency for these higher formulas. We also prove that $S O P_{2}$ is sufficient for a certain exact-saturation spectrum to be empty, connecting to work of Shelah [22] and Kaplan and Shelah 7]. The results seem persuasive and we believe they begin a structure theory for $\mathrm{NSOP}_{2}$ theories. The introductions to each section contain further motivation for these results and discussions of prior work.

Sections 5 and 8 may be read essentially independently of prior sections. Section 7 uses one earlier theorem; the reader interested in the $\triangleleft_{*}$ result may wish to begin there and refer back as needed.

\section{Contents}

1. Background on cofinality spectrum problems

1.1. New definitions: weak and hereditary CSPs. 
2. CSPs with exponentiation

3. On bounded arithmetic

4. Saturated models of Peano arithmetic

5. On the local cardinals $\mathfrak{p}_{\mathbf{s}, \mathbf{a}}$ and $\mathfrak{t}_{\mathbf{s}, \mathbf{a}}$

6. Characterizing the $\triangleleft^{*}$-maximal class 22

7. Useful tools and additional definitions 27

8. Towards a structure theory for $\mathrm{NSOP}_{2}$

References

\section{BACKGROUND ON COFINALITY SPECTRUM PROBLEMS}

Roughly speaking, a cofinality spectrum problem $\mathbf{s}=\left(M, M_{1}, M^{+}, M_{1}^{+}, \Delta\right)$ is given by:

- an elementary pair of models $\left(M, M_{1}\right)$ which can be expanded to the elementary pair $\left(M^{+}, M_{1}^{+}\right)$, and

- a set $\Delta$ of formulas in $\tau(M)$ defining discrete linear orders, closed under finite Cartesian products,

such that in the expanded model $M_{1}^{+}$,

- each instance of a formula in $\Delta$ defines a "pseudofinite" linear order, meaning that each of its $M_{1}^{+}$-definable subsets has a first and last element,

- on at least one of the Cartesian products, the ordering is well behaved, e.g. like the Gödel pairing function 1

and

- $\left(M^{+}, M_{1}^{+}, \Delta\right)$ has "enough set theory for trees," meaning essentially that for each $\varphi \in \Delta$ there is $\psi \in \tau\left(M_{1}^{+}\right)$so that for each linear order defined by an instance of $\varphi$, there is a tree defined by an instance of $\psi$ :

- whose nodes are functions from that order to itself, of length bounded by a distinguished element $d$ of the order, and

- the basic operations on this tree (the partial order on nodes given by initial segment, the length of a node i.e. size of its domain, the value of a node at an element of its domain, and concatenation of an additional value) are likewise uniformly definable.

Natural and motivating examples of cofinality spectrum problems may be constructed beginning with pairs of models of Peano arithmetic or pairs of models of set theory for $\left(M^{+}, M_{1}^{+}\right)$with $M$ being a reduct to a language containing linear order; or beginning with a pair $\left(M, M_{1}\right)$ where $M_{1}$ is an ultrapower of $M$, using the theorem that ultrapowers commute with reducts. See [8] $\S 2.3$ for more details on these examples. The fruitful idea was simply that, in some sufficiently rich model,

\footnotetext{
${ }^{1}$ Gödel's pairing function orders pairs of ordinals first by maximum, then by first coordinate, then by second coordinate. If e.g. there is a total linear order on the model, it makes sense to compare ("maximum") elements in any two such ordered sets; if not, this requirement may be satisfied by some $\mathbf{a} \times \mathbf{a}$, that is the Cartesian product of a given ordered set with itself.
} 
one may study the amount of saturation in an underlying linear order in relation to the fullness of the derived trees.

Here are the formal definitions, originally given in 8 .

Definition 1.1 (Enough set theory for trees, 8] Definition 2.3). Let $M_{1}$ be a model and $\Delta$ a nonempty set of formulas in the language of $M_{1}$. We say that $\left(M_{1}, \Delta\right)$ has enough set theory for trees when the following conditions are true.

(1) $\Delta$ consists of first-order formulas $\varphi(\bar{x}, \bar{y} ; \bar{z})$, with $\ell(\bar{x})=\ell(\bar{y})$.

(2) For each $\varphi \in \Delta$ and each parameter $\bar{c} \in{ }^{\ell(\bar{z})} M_{1}, \varphi(\bar{x}, \bar{y}, \bar{c})$ defines a discrete linear order on $\left\{\bar{a}: M_{1} \models \varphi(\bar{a}, \bar{a}, \bar{c})\right\}$ with a first and last element.

(3) The family of all linear orders defined in this way will be denoted $\operatorname{Or}\left(\Delta, M_{1}\right)$. Specifically, each $\mathbf{a} \in \operatorname{Or}\left(\Delta, M_{1}\right)$ is a tuple $\left(X_{\mathbf{a}}, \leq_{\mathbf{a}}, \varphi_{\mathbf{a}}, \bar{c}_{\mathbf{a}}, d_{\mathbf{a}}\right)$, where:

(a) $X_{\mathbf{a}}$ denotes the underlying set $\left\{\bar{a}: M_{1} \models \varphi_{\mathbf{a}}\left(\bar{a}, \bar{a}, \bar{c}_{\mathbf{a}}\right)\right\}$

(b) $\bar{x} \leq_{\mathbf{a}} \bar{y}$ abbreviates the formula $\varphi_{\mathbf{a}}\left(\bar{x}, \bar{y}, \bar{c}_{\mathbf{a}}\right)$

(c) $d_{\mathbf{a}} \in X_{\mathbf{a}}$ is a bound for the length of elements in the associated tree; it is often, but not always, $\max X_{\mathbf{a}}$. If $d_{\mathbf{a}}$ is finite, we call a trivial.

(4) For each $\mathbf{a} \in \operatorname{Or}(\mathbf{a}),\left(X_{\mathbf{a}}, \leq_{\mathbf{a}}\right)$ is pseudofinite, meaning that any bounded, nonempty, $M_{1}$-definable subset has $a \leq_{\mathbf{a}}$-greatest and $\leq_{\mathbf{a}}$-least element.

(5) For each pair $\mathbf{a}$ and $\mathbf{b}$ in $\operatorname{Or}\left(\Delta, M_{1}\right)$, there is $\mathbf{c} \in \operatorname{Or}\left(\Delta, M_{1}\right)$ such that:

(a) there exists an $M_{1}$-definable bijection $\operatorname{Pr}: X_{\mathbf{a}} \times X_{\mathbf{b}} \rightarrow X_{\mathbf{c}}$ such that the coordinate projections are $M_{1}$-definable.

(b) if $d_{\mathbf{a}}$ is not finite in $X_{\mathbf{a}}$ and $d_{\mathbf{b}}$ is not finite in $X_{\mathbf{b}}$, then also $d_{\mathbf{c}}$ is not finite in $X_{\mathbf{c}}$.

(6) For some nontrivial $\mathbf{a} \in \operatorname{Or}\left(\Delta, M_{1}\right)$, there is $\mathbf{c} \in \operatorname{Or}\left(\Delta, M_{1}\right)$ such that $X_{\mathbf{c}}=\operatorname{Pr}\left(X_{\mathbf{a}} \times X_{\mathbf{a}}\right)$ and the ordering $\leq_{\mathbf{c}}$ satisfies:

$M_{1} \models\left(\forall x \in X_{\mathbf{a}}\right)\left(\exists y \in X_{\mathbf{c}}\right)\left(\forall x_{1}, x_{2} \in X_{\mathbf{a}}\right)\left(\max \left\{x_{1}, x_{2}\right\} \leq_{\mathbf{a}} x \Longleftrightarrow \operatorname{Pr}\left(x_{1}, x_{2}\right) \leq_{\mathbf{c}} y\right)$

(7) To the family of distinguished orders, we associate a family of trees, as follows. For each formula $\varphi(\bar{x}, \bar{y} ; \bar{z})$ in $\Delta$ there are formulas $\psi_{0}, \psi_{1}, \psi_{2}$ of the language of $M_{1}$ such that for any $\mathbf{a} \in \operatorname{Or}(\mathbf{s})$ with $\varphi_{\mathbf{a}}=\varphi$ :

(a) $\psi_{0}\left(\bar{x}, \bar{c}_{\mathbf{a}}\right)$ defines a set, denoted $\mathcal{T}_{\mathbf{a}}$, of functions from $X_{\mathbf{a}}$ to $X_{\mathbf{a}}$.

(b) $\psi_{1}(\bar{x}, \bar{y}, \bar{c})$ defines a function $\lg _{\mathbf{a}}: \mathcal{T}_{\mathbf{a}} \rightarrow X_{\mathbf{a}}$ satisfying:

(i) for all $b \in \mathcal{T}_{\mathbf{a}}, \lg _{\mathbf{a}}(b) \leq_{\mathbf{a}} d_{\mathbf{a}}$.

(ii) for all $b \in \mathcal{T}_{\mathbf{a}}, \lg _{\mathbf{a}}(b)=\max (\operatorname{dom}(b))$.

(c) $\psi_{2}(\bar{x}, \bar{y}, \bar{c})$ defines a function from $\left\{(b, a): b \in \mathcal{T}_{\mathbf{a}}, a \in X_{\mathbf{a}}, a<\mathbf{a} \lg _{\mathbf{a}}(b)\right\}$ into $X_{\mathbf{a}}$ whose value is called $\operatorname{val}_{\mathbf{a}}(b, c)$, and abbreviated $b(a)$.

(i) if $c \in \mathcal{T}_{\mathbf{a}}$ and $\lg _{\mathbf{a}}(c)<d_{\mathbf{a}}$ and $a \in X_{\mathbf{a}}$, then $c^{\frown}\langle a\rangle$ exists, i.e. there is $c^{\prime} \in \mathcal{T}_{\mathbf{a}}$ such that $\lg _{\mathbf{a}}\left(c^{\prime}\right)=\lg _{\mathbf{a}}(c)+1, c^{\prime}\left(\lg _{\mathbf{a}}(c)\right)=a$, and

$$
\left(\forall a<_{\mathbf{a}} \lg _{\mathbf{a}}(c)\right)\left(c(a)=c^{\prime}(a)\right)
$$

(ii) $\psi_{0}(\bar{x}, \bar{c})$ implies that if $b_{1} \neq b_{2} \in \mathcal{T}_{\mathbf{a}}, \lg _{\mathbf{a}}\left(b_{1}\right)=\lg _{\mathbf{a}}\left(b_{2}\right)$ then for some $n<_{\mathbf{a}} \lg _{\mathbf{a}}\left(b_{1}\right), b_{1}(n) \neq b_{2}(n)$.

(d) $\psi_{3}(\bar{x}, \bar{y}, \bar{c})$ defines the partial order $\triangleleft_{\mathbf{a}}$ on $\mathcal{T}_{\mathbf{a}}$ given by initial segment, that is, such that that $b_{1} \triangleleft_{\mathbf{a}} b_{2}$ implies:

(i) for all $b, c \in \mathcal{T}_{\mathbf{a}}, b \triangleleft c$ implies $\lg _{\mathbf{a}}(b) \leq_{\mathbf{a}} \lg _{\mathbf{a}}(c)$. 
(ii) $\lg _{\mathbf{a}}\left(b_{1}\right) \leq_{\mathbf{a}} \lg _{\mathbf{a}}\left(b_{2}\right)$

(iii) $\left(\forall a<_{\mathbf{a}} \lg _{\mathbf{a}}\left(b_{1}\right)\right)\left(b_{2}(a)=b_{1}(a)\right)$

The family of all $\mathcal{T}_{\mathbf{a}}$ defined this way will be denoted $\operatorname{Tr}\left(\Delta, M_{1}\right)$. We refer to elements of this family as trees.

Notice the pairing requirement in condition (6). While we need Cartesian products to exist, it is largely unimportant what exactly the order on these products is (as long as it satisfies the other requirements). It's sufficient for the results of [8] that one such order behave well, like the usual Gödel pairing function. In the course of the present paper, we will consider weakenings of this requirement.

Definition 1.2 (Cofinality spectrum problems, 8] Definition 2.4). We call the six-tuple

$$
\mathbf{s}=\left(M, M_{1}, M^{+}, M_{1}^{+}, T, \Delta\right)
$$

$a$ cofinality spectrum problem when:

(1) $M \preceq M_{1}$.

(2) $T \supseteq T h(M)$ is a theory in a possibly larger vocabulary.

(3) $\Delta$ is a set of formulas in the language of $M$, i.e., we are interested in studying the orders of $M, M_{1}$ in the presence of the additional structure of the expansion.

(4) $M^{+}, M_{1}^{+}$expand $M, M_{1}$ respectively so that $M^{+} \preceq M_{1}^{+} \models T$ and $\left(M_{1}^{+}, \Delta\right)$ has enough set theory for trees.

(5) We may refer to the components of $\mathbf{s}$ as $M^{\mathbf{s}}, \Delta^{\mathbf{s}}$, etc. for definiteness. When $T=T h(M), M=M^{+}, M_{1}=M_{1}^{+}$, or $\Delta$ is the set of all formulas $\varphi(x, y, \bar{z})$ in the language of $T$ which satisfy $1.1(2)-(4)$, these may be omitted.

Remark 1.3. The identities of $M^{+}$and $M_{1}$ are not essential to many arguments.

Definition 1.4 (The cardinals $\mathfrak{p}_{\mathbf{s}}, \mathfrak{t}_{\mathbf{s}}$ and the cut spectrum, [8] Definition 2.8). For a cofinality spectrum problem $\mathbf{s}$ we define the following:

(1) $\operatorname{Or}(\mathbf{s})=\operatorname{Or}\left(\Delta^{\mathbf{s}}, M_{1}^{\mathbf{s}}\right)$

(2) $\mathcal{C}^{\mathrm{ct}}(\mathbf{s})=\left\{\left(\kappa_{1}, \kappa_{2}\right)\right.$ : for some $\mathbf{a} \in \operatorname{Or}(\mathbf{s}),\left(X_{\mathbf{a}}, \leq_{\mathbf{a}}\right)$ has a $\left(\kappa_{1}, \kappa_{2}\right)$-cut $\}$. Note that the $\kappa_{\ell}$ are infinite.

(3) $\operatorname{Tr}(\mathbf{s})=\left\{\mathcal{T}_{\mathbf{a}}: \mathbf{a} \in \operatorname{Or}(\mathbf{s})\right\}=\operatorname{Tr}\left(\Delta^{\mathbf{s}}, M_{1}^{\mathbf{s}}\right)$

(4) $\mathcal{C}^{\text {ttp }}(\mathbf{s})=\left\{\kappa: \kappa \geq \aleph_{0}, \mathbf{a} \in \operatorname{Or}(\mathbf{s})\right.$, and there is in the tree $\mathcal{T}_{\mathbf{a}}$ a strictly increasing sequence of cofinality $\kappa$ with no upper bound $\}$

(5) Let $\mathfrak{t}_{\mathbf{s}}$ be $\min \mathcal{C}^{\mathrm{ttp}}(\mathbf{s})$ and let $\mathfrak{p}_{\mathbf{s}}$ be $\min \left\{\kappa:\left(\kappa_{1}, \kappa_{2}\right) \in \mathcal{C}^{\mathrm{ct}}(\mathbf{s})\right.$ and $\left.\kappa=\kappa_{1}+\kappa_{2}\right\}$.

A key role will be played by $\mathcal{C}\left(\mathbf{s}, \mathfrak{t}_{\mathbf{s}}\right)$, where this means:

(6) For $\lambda$ an infinite cardinal, write

$$
\mathcal{C}(\mathbf{s}, \lambda)=\left\{\left(\kappa_{1}, \kappa_{2}\right): \kappa_{1}+\kappa_{2}<\lambda,\left(\kappa_{1}, \kappa_{2}\right) \in \mathcal{C}^{\mathrm{ct}}(\mathbf{s})\right\} .
$$


By definition, both $\mathfrak{t}_{\mathbf{s}}$ and $\mathfrak{p}_{\mathbf{s}}$ are regular. The main engine of the paper [8] was the following theorem, proved by model-theoretic means. Note that it entails $\mathfrak{t}_{\mathrm{s}} \leq \mathfrak{p}_{\mathrm{s}}$

Theorem A ([8] Theorem 9.1). For any cofinality spectrum problem $\mathbf{s}, \mathcal{C}\left(\mathbf{s}, \mathfrak{t}_{\mathbf{s}}\right)=\emptyset$.

Discussion 1.5. We now resume the discussion following Definition 1.1. In [8], Cartesian products were used in two ways. First, we needed the simple existence of Cartesian products of pairs of elements of $\operatorname{Or}(\mathbf{s})$, with no restrictions on the ordering of the pairs other than: (i) pseudofiniteness and (ii) the property that if $d_{\mathbf{a}}, d_{\mathbf{b}}$ are nonstandard then so is $d_{\mathbf{a} \times \mathbf{b}}$ (call such a product nontrivial). This was needed for the basic arguments connecting behavior across all orders: notably, establishing existence of the lower cofinality function. To rule out symmetric cuts, we needed only to be able to take the Cartesian product of an order with itself. For the main lemma ruling out antisymmetric cuts, we needed (a) the existence of $\mathbf{a}$ and $\mathbf{a}^{\prime}$ such that $\mathbf{a}$ is coverable as a pair by $\mathbf{a}^{\prime}$ (see below), and then (b) the existence of the nontrivial product $\mathbf{a} \times \mathbf{a} \times \mathbf{a} \times \mathbf{a}^{\prime} \times \mathbf{a}^{\prime} \times \mathbf{a}^{\prime}$. One may take as a definition of "coverable as a pair" its key property, Corollary 5.7(a) of [8] [quoted here with the assumptions of nontriviality made there explicitly added in]:

(**) there is $a \in X_{\mathbf{a}}$, with $a<d_{\mathbf{a}}$ and $a$ not a finite successor of $0_{\mathbf{a}}$, such that the Gödel codes for functions from $[0, a]_{\mathbf{a}}$ to $[0, a]_{\mathbf{a}} \times[0, a]_{\mathbf{a}}$ may be definably identified with a definable subset of $X_{\mathbf{a}^{\prime}}$ whose greatest element is $<d_{\mathbf{a}^{\prime}}$.

For property $\left({ }^{* *}\right)$, it suffices to have $\mathbf{a} \in \operatorname{Or}(\mathbf{s})$ such that the order on $\mathbf{a} \times \mathbf{a}$ is given by the Gödel pairing function and $d_{\mathbf{a} \times \mathbf{a}}$ is nonstandard, by the arguments of [8]. We will give some alternate sufficient conditions in Section 7

The following summarizes our conventions.

Convention 1.6 (Key conventions on CSPs).

(a) Recall that definable means in the sense of $M_{1}^{+}=M_{1}^{+}[\mathbf{s}]$, unless otherwise stated $2^{2}$

(b) Recall that for $\mathcal{T}_{\mathbf{a}} \in \operatorname{Tr}(\mathbf{s}), \eta \in \mathcal{T}_{\mathbf{a}}$ implies that $\lg (\eta) \leq d_{\mathbf{a}}$, and we have closure under concatenation, i.e. if $\lg (\eta)<d_{\mathbf{a}}$ and $a \in X_{\mathbf{a}}$ then $\eta \frown\langle a\rangle \in \mathcal{T}_{\mathbf{a}}$.

(c) When $\mathcal{T}$ is a definable subtree of $\mathcal{T}_{\mathbf{a}}$, we will write

$$
\left(\mathcal{T}, \triangleleft_{\mathbf{a}}\right) \text { or just }(\mathcal{T}, \triangleleft) \text { to mean }\left(\mathcal{T}, \triangleleft_{\mathbf{a}}\lceil\mathcal{T})\right.
$$

(d) When $\eta$ is a sequence of $n$-tuples, $t<\lg (\eta)$, and $k<n$, write $\eta(t, k)$ for the $k$ th element of $\eta(t)$.

(e) Generalizing [8], we use "internal map" in the present paper for any map definable in $M_{1}^{+}$, not necessarily an element of some $\mathcal{T} \in \operatorname{Tr}(\mathbf{s})$.

(f) We call a definable, discrete order pseudofinite when every nonempty, bounded, definable subset has a first and last element.

(g) We call $\mathbf{a} \in \operatorname{Or}(\mathbf{s})$ nontrivial when $d_{\mathbf{a}}$ is not a finite successor of $0_{\mathbf{a}}$, and we call $\mathbf{s}$ nontrivial when at least one $\mathbf{a} \in \operatorname{Or}(\mathbf{s})$ is nontrivial.

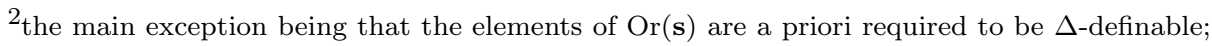
this is relaxed if $\mathbf{s}$ is hereditarily closed, defined below.
} 
1.1. New definitions: weak and hereditary CSPs. We now give some new definitions weakening and extending CSPs looking towards applications.

First, as regards applying CSPs it will be useful to keep track of whether or not the assumption on closure under Cartesian products is needed. For example, in dealing with a CSP arising from a model $M=M^{+}=M_{1}^{+}$whose domain is linearly ordered, it may be that we care primarily about cuts occurring in the linear order $\operatorname{dom}(M)$. Thus, we introduce 'weak' CSPs in Definition 1.7 and in the present paper, use them where possible.

\section{Definition 1.7 (Weak CSP).}

(1) Call $\mathbf{s}$ a weak CSP if it satisfies 1.2 omitting the requirement that $\operatorname{Or}(\mathbf{s})$ be closed under Cartesian product, $1.1(5)+(6)$.

(2) For $\mathbf{s}$ a weak CSP, $\mathbf{a} \in \operatorname{Or}(\mathbf{s})$, let $Y_{\mathbf{a}}=Y_{\mathbf{a}, \mathbf{s}}=\left\{a \in X_{\mathbf{a}}: a<_{\mathbf{a}} d_{\mathbf{a}}\right\}$.

Second, in 8] Convention 5.1 we had observed that CSPs have available an internal notion of cardinality $3^{3}$ In the present paper, we use the more general definition:

Definition 1.8. Let $\mathbf{s}$ be a cofinality spectrum problem. Whenever $A, B$ are definable subsets of $M_{1}$ (with parameters), we write

$$
\text { " } A|\leq| B \mid "
$$

to mean "there exists an $M_{1}^{+}$-definable partial 1-to-1 function $h$ with $A \subseteq \operatorname{dom}(h)$ and range $(h) \subseteq B$ ". Likewise, we write

$$
\text { " } A|<| B \mid "
$$

to mean " $(|A| \leq|B|) \wedge \neg(|B| \leq|A|)$," i.e. $|A| \leq|B|$ and there does not exist an $M_{1}^{+}$-definable injection from $B$ into $A$.

Third, while the definition of CSP carefully lays out the 'canonical' orders and trees, it is natural to add some internal closure conditions, stating essentially that objects which are internally isomorphic to canonical ones also count.

Definition 1.9. Let $\mathbf{s}$ be a CSP or weak CSP.

(1) We call $\mathbf{b}$ a pseudo-order of $\mathbf{s}$ and write $\mathbf{b} \in \operatorname{PsOr}(\mathbf{s})$ when it satisfies all of the requirements on elements of $\operatorname{Or}(\mathbf{s})$ from Definition 1.1 in the case where $\Delta$ is the set of all formulas in the language. 47 (The point is that possibly the formulas defining it are not from $\Delta_{\mathbf{s}}$.)

(2) Given $\mathbf{b}_{2} \in \operatorname{PsOr}(\mathbf{s})$, we say $\mathbf{b}_{1}$ is a suborder of $\mathbf{b}_{2}$ and write $\mathbf{b}_{1} \in \operatorname{Sub}\left(\mathbf{b}_{2}\right)$ when: $\mathbf{b}_{1} \in \operatorname{PsOr}(\mathbf{s}), X_{\mathbf{b}_{1}} \subseteq X_{\mathbf{b}_{2}}$ as linear orders, and there is $d \leq_{\mathbf{b}_{2}} d_{\mathbf{b}_{2}}$

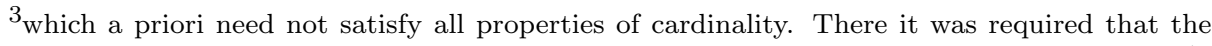
function be an element of the tree $\mathcal{T}_{\mathbf{a} \times \mathbf{b}}$. Depending on the amount of set theory available in $M_{1}^{+}$, the present weakening is an a priori loss in definability: when functions are required to belong to a definable tree, we are always able to quantify over them, whereas in the definition below the quantification over all such functions may be strictly informal (not first order).

${ }^{4}$ In particular, it must have an associated, definable tree, with definable length functions and concatenation, and so forth.
} 
and $\eta \in \mathcal{T}_{\mathbf{b}_{2}}$ with $\max \operatorname{dom}(\eta)=d=d_{\mathbf{b}_{1}}$ which lists $\left\langle c \in X_{\mathbf{b}_{1}}: c<_{\mathbf{b}_{1}} d_{\mathbf{b}_{1}}\right\rangle$ in increasing order $5^{5}$

Definition 1.10 (Hereditary closure). Let $\mathbf{s}$ be a CSP or weak CSP.

(1) Let $\mathbf{b}_{1}, \mathbf{b}_{2} \in \operatorname{PsOr}(\mathbf{s})$. We say $(f, g, h)$ is an accurate isomorphism from $\mathbf{b}_{1}$ onto $\mathbf{b}_{2}$ when it respects both the order structure below $d_{\mathbf{b}_{i}}$ and the tree structure, that is:

(a) $f$ is a one-to-one mapping, definable in $M_{1}^{+}$, from $X_{\mathbf{b}_{1}}$ to $X_{\mathbf{b}_{2}}$

(b) $g$ is a one-to-one mapping, definable in $M_{1}^{+}$, from $\left\{a: a<_{\mathbf{b}_{1}} d_{\mathbf{b}_{1}}\right\}$ onto $\left\{a: a<_{\mathbf{b}_{2}} d_{\mathbf{b}_{2}}\right\}$, such that $a<_{\mathbf{b}_{1}} c<_{\mathbf{b}_{1}} d_{\mathbf{b}_{1}} \Longleftrightarrow g(a)<_{\mathbf{b}_{2}}$ $g(c)<_{\mathbf{b}_{2}} d_{\mathbf{b}_{2}}$.

(c) $h$ is a one-to-one function definable in $M_{1}^{+}$from $\mathcal{T}_{\mathbf{b}_{1}}$ onto $\mathcal{T}_{\mathbf{b}_{2}}$ such that

$$
\eta \triangleleft \mathcal{T}_{\mathbf{b}_{1}} \nu \Longleftrightarrow h(\eta)<\mathcal{T}_{\mathbf{b}_{2}} h(\nu)
$$

and $\eta=\nu^{\wedge}\langle a\rangle$ in $\mathbf{b}_{1}$ iff $h(\eta)=h(\nu)^{\wedge}\langle f(a)\rangle$ in $\mathbf{b}_{2}$.

(2) Call $\mathbf{s}$ hereditary if $\mathrm{Or}(\mathbf{s})$ is closed under Sub and accurate isomorphism.

(3) Say " $\mathbf{s}_{2}$ is the hereditary closure of $\mathbf{s}_{1}$ " when $\mathbf{s}_{1}, \mathbf{s}_{2}$ are the same except that $\operatorname{Or}\left(\mathbf{s}_{2}\right)$ is the closure of $\operatorname{Or}\left(\mathbf{s}_{1}\right)$ under members of Sub and under accurate isomorphism.

Observation 1.11. If $\mathbf{s}$ is a weak CSP then so is its hereditary closure.

Observation 1.12. As a consequence of Definition 1.9(3), if $\mathbf{s}$ is a weak CSP which is hereditarily closed, then for any $\mathbf{a} \in \operatorname{Or}(\mathbf{s})$, any $X \subseteq Y_{\mathbf{a}}$ and any $d \in X \cap\left[0_{\mathbf{a}}, d_{\mathbf{a}}\right]$, there is $\mathbf{b} \in \operatorname{Or}(\mathbf{s})$ with $X_{\mathbf{b}}=X,<_{\mathbf{b}}=<_{\mathbf{a}} \mid X$, and $d=d_{\mathbf{b}}$.

\section{CSPS WITH EXPONENTIATION}

A feature of CSPs is that one can always develop a certain amount of Peano arithmetic internally, as in [8] $\S 5$. That observation motivates the main definition of this section, 'closure under exponentiation.' In this section we work out that CSPs of this kind have the very nice property that $\mathfrak{p}_{\mathbf{s}}=\mathfrak{t}_{\mathbf{s}}$. For context, recall that the main theorem of $\left[8\right.$ that $\mathcal{C}\left(\mathbf{s}, \mathfrak{t}_{\mathbf{s}}\right)=\emptyset$ only implies that $\mathfrak{t}_{\mathbf{s}} \leq \mathfrak{p}_{\mathbf{s}}$. For the other direction, it's natural to try to show that a $\kappa$-indexed path through some $\mathcal{T}$ with no upper bound translates to a $(\kappa, \kappa)$-cut in some $\mathbf{a} \in \operatorname{Or}(\mathbf{s})$. The problem is that the natural translation produces a definable, discrete linear order which contains a $(\kappa, \kappa)$-cut but is not necessarily a member of $\operatorname{Or}(\mathbf{s})$. After explaining the problem, we will propose the solution.

Hypothesis 2.1. In this section, unless otherwise stated, $\mathrm{s}$ is a weak CSP. For transparency, we assume $\mathbf{s}$ is hereditary, i.e. closed under Sub and full internal isomorphism. We retain the notation $Y_{\mathbf{a}}$ from 1.7 .

\footnotetext{
${ }^{5}$ So e.g. $\mathbf{b}_{1} \notin \operatorname{Sub}\left(\mathbf{b}_{2}\right)$ when $X_{\mathbf{b}_{1}}$ is an initial segment of $X_{\mathbf{b}_{2}}$ and $d_{\mathbf{b}_{2}}<\max \left(X_{\mathbf{b}_{1}}\right)$, since there is not enough room for the list.
} 
Definition 2.2. (Flattening the tree, c.f. 8] Lemma 6.2) Let $\mathbf{a} \in \operatorname{Or}(\mathbf{s})$ be given. We define the following linear order. Fix in advance two distinct elements of $X_{\mathbf{a}}$; without loss of generality ${ }^{6}$ we use $0_{\mathbf{a}}, 1_{\mathbf{a}}$, called 0,1 , so $0<_{\mathbf{a}} 1$. Let $\mathcal{S}_{\mathbf{a}}$ be the set $\mathcal{T}_{\mathbf{a}} \times\{0,1\}$. Let $<_{\mathcal{S}_{\mathbf{a}}}$ be the linear order on $\mathcal{S}_{\mathbf{a}}$ defined as follows:

- If $c=d$, then $(c, i)<_{\mathcal{S}_{\mathbf{a}}}(d, j)$ iff $i<_{\mathbf{a}} j$

- If $c \triangleleft_{\mathbf{a}} d$ and $c \neq d$, then $(c, 0)<_{\mathcal{S}_{\mathbf{a}}}(d, 0)<\mathcal{S}_{\mathbf{a}}(d, 1)<\mathcal{S}_{\mathbf{a}}(c, 1)$

- If $c, d$ are $\triangleleft$-incomparable, then let $e \in \mathcal{T}_{\mathbf{a}}, n_{c}, n_{d} \in \mathcal{S}_{\mathbf{a}}$ be such that $e=$ $\operatorname{cis}(c, d)$ is the common initial segment and $e^{\frown} n_{c} \triangleleft_{\mathbf{a}} c$ and $e^{\frown} n_{d} \triangleleft_{\mathbf{a}} d$. Necessarily $n_{c} \neq n_{d}$ by definition of $e$, so for $s, t \in\{0,1\}$ we define

$$
(c, s)<_{\mathcal{S}_{\mathbf{a}}}(d, t) \Longleftrightarrow n_{c}<_{\mathbf{a}} n_{d}
$$

For each given $\mathbf{a}$, we refer to this ordered $\operatorname{set}\left(\mathcal{S}_{\mathbf{a}},<_{\mathcal{S}_{\mathbf{a}}}\right)$ just constructed as "the order given by flattening the tree $\mathcal{T}_{\mathbf{a}}$ ".

Convention 2.3. For the remainder of this article, given $\mathbf{s}$ and $\mathbf{a} \in \operatorname{Or}(\mathbf{s})$, let $\left(\mathcal{S}_{\mathbf{a}},<_{\mathcal{S}_{\mathbf{a}}}\right)$ denote the order constructed in 2.2

Fact 2.4. Let $\mathbf{s}$ be a weak CSP. If $\mathbf{a} \in \operatorname{Or}(\mathbf{s}), \mathcal{T}_{\mathbf{a}}$ witnesses treetops and $\left(\mathcal{S}_{\mathbf{a}},<_{\mathcal{S}_{\mathbf{a}}}\right)$ is the order given by flattening the tree $\mathcal{T}_{\mathbf{a}}$ then $\left(\mathcal{S}_{\mathbf{a}},<_{\mathcal{S}_{\mathbf{a}}}\right)$ has a $\left(\mathfrak{t}_{\mathbf{s}}, \mathfrak{t}_{\mathbf{s}}\right)$-cut.

Proof. The proof when $\mathbf{s}$ is a CSP is [8] Lemma 6.2. No assumptions are made about closure under Cartesian products in that proof, so the identical result holds for weak CSPs as well.

We cannot a priori conclude from Fact 2.4 that $\left(\mathfrak{t}_{\mathbf{s}}, \mathfrak{t}_{\mathbf{s}}\right) \in \mathcal{C}\left(\mathbf{s}, \mathfrak{t}_{\mathbf{s}}\right)$, because we have not shown that $\mathcal{S}_{\mathbf{a}}$ belongs to $\operatorname{Or}(\mathbf{s})$. However, note that $\left(\mathcal{S}_{\mathbf{a}},<_{\mathcal{S}_{\mathbf{a}}}\right)$ is a definable discrete linear order with a first and last element, since:

Observation 2.5. For any weak cofinality spectrum problem $\mathbf{s}$ and $\mathbf{a} \in \operatorname{Or}(\mathbf{s})$, there is an internal order-isomorphism between an initial segment of $\left(\mathcal{S}_{\mathbf{a}},<_{\mathcal{S}_{\mathbf{a}}}\right)$ and $Y_{\mathbf{a}}$.

Proof. Let $\left\langle c_{\alpha}: \alpha<d_{\mathbf{a}}\right\rangle$ be the sequence of elements of $\mathcal{T}_{\mathbf{a}}$ corresponding to functions which are constantly $0_{\mathbf{a}}$, listed in increasing order: this sequence is linearly ordered by $\triangleleft_{\mathbf{a}}$. By the definition of $\mathcal{S}_{\mathbf{a}}$, we have that $\left(\left\{\left(c_{\alpha}, 0\right): \alpha<d_{\mathbf{a}}\right\},<_{\mathbf{a}}\right)$ is an initial segment of $\left(\mathcal{S}_{\mathbf{a}},<_{\mathcal{S}_{\mathbf{a}}}\right)$. Moreover, it is isomorphic to $\left(Y_{\mathbf{a}},<_{\mathbf{a}}\right)$ via the internal map $\lg _{\mathbf{a}}$ from Definition 1.1(7).

Definition 2.6 (Closed under exponentiation). Let $\mathbf{s}$ be a weak CSP. We say $\mathbf{s}$ is closed under (simple) exponentiation when for every nontrivial $\mathbf{a} \in \operatorname{Or}(\mathbf{s})$ there is a nontrivial $\mathbf{b} \in \operatorname{Or}(\mathbf{s})$ such that $\left(X_{\mathbf{b}},<_{\mathbf{b}}\right)$ and $\left(\mathcal{S}_{\mathbf{a}},<_{\mathcal{S}_{\mathbf{a}}}\right)$ are internally isomorphic.

Definition 2.7. For $\mathbf{s}$ a weak CSP, it will also be useful to define:

(a) $\mathbf{s}$ is strongly closed under exponentiation, or has strong exponentiation, when: for every $\mathbf{a} \in \operatorname{Or}(\mathbf{s})$ there is $\mathbf{b} \in \operatorname{Or}(\mathbf{s})$ such that $\left(\mathcal{S}_{\mathbf{a}},<_{\mathcal{S}_{\mathbf{a}}}\right)$ is accurately isomorphic to $\left(X_{\mathbf{b}}\left\lceil d_{\mathbf{b}},<_{\mathbf{b}}\right)\right.$, following Definition 1.9 . 7

\footnotetext{
${ }^{6}$ recall that $X_{\mathbf{a}}$ is a discrete linear order with first element, so $0_{\mathbf{a}}, 1_{\mathbf{a}}$ have the natural meanings.

${ }^{7}$ In Definition 1.9 accurate isomorphism of two linear orders $\mathbf{b}_{0}, \mathbf{b}_{1}$ involves an isomorphism of sets plus an order-isomorphism below the bounds $d_{\mathbf{b}_{i}}$. For these purposes, consider the $d$ for $S_{\mathbf{a}}$ to be $\max S_{\mathbf{a}}$.
} 
(b) $\mathbf{s}$ is weakly closed under exponentiation, or has weak exponentiation, when: for every $\mathbf{a} \in \operatorname{Or}(\mathbf{s})$ there is $\mathbf{b} \in \operatorname{Or}(\mathbf{s})$ such that $\left(X_{\mathbf{b}},<_{\mathbf{b}}\right)$ and $\left(\mathcal{S}_{\mathbf{a}},<_{\mathcal{S}_{\mathbf{a}}}\right)$ are internally isomorphic and $\left(Y_{\mathbf{b}},<_{\mathbf{b}}\right),\left(Y_{\mathbf{a}},<_{\mathbf{a}}\right)$ are internally isomorphic.

Discussion 2.8. The phrase "s has exponentiation" clearly covers 2.6, 2.7 (a) and $2.7(b)$. One of the issues raised by 2.7 is whether $\left(\mathcal{S}_{\mathbf{a}},<_{\mathcal{S}_{\mathbf{a}}}\right)$ may map onto $X_{\mathbf{b}}$ in such a way that $d_{\mathbf{b}}$ is not above the range of the map. To distinguish 2.6 from 2.7. we will say "simple exponentiation."

Corollary 2.9. Let $\mathbf{s}$ be a weak cofinality spectrum problem which has exponentiation, $\mathbf{a} \in \operatorname{Or}(\mathbf{s})$. Then:

(1) $\left(\mathcal{S}_{\mathbf{a}},<_{\mathcal{S}_{\mathbf{a}}}\right)$ is a discrete linear order in which every nonempty definable subset has a first and last element.

(2) There is $\mathbf{b} \in \operatorname{Or}(\mathbf{s})$ so that $\left(X_{\mathbf{b}}, \leq_{\mathbf{b}}\right)$ has a $\left(\mathfrak{t}_{\mathbf{s}}, \mathbf{t}_{\mathbf{s}}\right)$-cut.

Proof. (1) This is inherited from the order-isomorphism to an element of $\operatorname{Or}(\mathbf{s})$.

(2) Let $\mathcal{T}_{\mathbf{a}}$ witness treetops. By Fact 2.4, the order $\left(\mathcal{S}_{\mathbf{a}},<_{\mathcal{S}_{\mathbf{a}}}\right)$ has a $\left(\mathfrak{t}_{\mathbf{s}}, \mathfrak{t}_{\mathbf{s}}\right)$-cut. Given $\mathbf{b} \in \operatorname{Or}(\mathbf{s})$ such that $\left(\mathcal{S}_{\mathbf{a}},<_{\mathcal{S}_{\mathbf{a}}}\right)$ is order-isomorphic to $X_{\mathbf{b}}$, clearly $\left(X_{\mathbf{b}}, \leq_{\mathbf{b}}\right)$ has a $\left(\mathfrak{t}_{\mathbf{s}}, \mathfrak{t}_{\mathbf{s}}\right)$-cut.

We arrive at a fact which will be useful throughout the paper: if $\mathbf{s}$ has exponentiation then $\mathfrak{p}_{\mathbf{s}}=\mathfrak{t}_{\mathbf{s}}$ and 'the first cut is symmetric'.

\section{Theorem 2.10.}

(1) Let $\mathbf{s}$ be a weak CSP with exponentiation. Then $\mathfrak{p}_{\mathbf{s}} \leq \mathfrak{t}_{\mathbf{s}}$.

(2) Let $\mathbf{s}$ be a CSP with exponentiation. Then $\mathfrak{p}_{\mathbf{s}}=\mathfrak{t}_{\mathbf{s}}$.

(3) Let $\mathbf{s}$ be a CSP with exponentiation. Then

$$
\mathbf{t}_{\mathbf{s}}=\min \left\{\kappa:(\kappa, \kappa) \in \mathcal{C}^{\mathrm{ct}}(\mathbf{s})\right\}
$$

and the first cut in $\mathcal{C}^{\mathrm{ct}}(\mathbf{s})$ is necessarily symmetric, that is, if

$$
\mu=\min \left\{\kappa+\lambda:(\kappa, \lambda) \in \mathcal{C}^{\mathrm{ct}}(\mathbf{s})\right\}
$$

then $(\mu, \mu) \in \mathcal{C}^{\text {ct }}(\mathbf{s})$.

Proof. First we prove (1). Corollary 2.9 (2) shows that if $\mathfrak{t}_{\mathbf{s}}=\kappa$, then $(\kappa, \kappa) \in \mathcal{C}^{\text {ct }}(\mathbf{s})$, so $\mathfrak{p}_{\mathbf{s}} \leq \kappa+\kappa=\kappa$. Thus $\mathfrak{p}_{\mathbf{s}} \leq \mathfrak{t}_{\mathbf{s}}$.

When in addition $\mathbf{s}$ is a cofinality spectrum problem the analysis of [8] applies. By Theorem A, $\$ 1$ above we have that $\mathcal{C}\left(\mathbf{s}, \mathfrak{t}_{\mathbf{s}}\right)=\emptyset$, thus $\mathfrak{t}_{\mathbf{s}} \leq \mathfrak{p}_{\mathbf{s}}$, proving (2).

For $(3)$, the proof of 2.10 shows that $\mathfrak{p}_{\mathbf{s}}=\min \left\{\kappa:(\kappa, \kappa) \in \mathcal{C}^{\text {ct }}(\mathbf{s})\right\}$. Since $\mathfrak{p}_{\mathbf{s}}=\mathbf{t}_{\mathbf{s}}$, this is sufficient.

However, as we will see in Theorem 5.3. the situation for the local versions of these cardinals, Definition 5.1 is more subtle. It would be interesting to explore this further. 


\section{ON BOUNDED ARITHMETIC}

In this section, working towards our first main application in $\$ 4$, we set up CSPs and weak CSPs arising from models of PA or BPA and check when they are closed under exponentiation in the sense just described.

Definition 3.1. A formula is called bounded if all of its quantifiers are bounded. By BPA we mean bounded Peano arithmetic, that is, the restriction of the Peano axioms containing induction only for bounded formulas.

When working with models of PA or BPA, we will use the notation $x^{y}$ in accordance with:

Fact 3.2. (Gaifman and Dimitracopoulos [3], see [14 §1.1) Let $I \Delta_{0}$ denote basic arithmetic with bounded induction. There exists a $\Delta_{0}$ formula $\varphi(x, y, z)$, which we denote by $x^{y}=z$, which can be shown in $I \Delta_{0}$ to have all the usual properties of the graph of exponentiation except for the sentence

$$
\forall x \forall y \exists z\left(x^{y}=z\right) .
$$

Regarding Definition 3.3 we will focus on $\mathbf{s}_{3}[N]$ for $N$ a model of PA or BPA.

Definition 3.3 (Some canonical weak CSPs from models). Let $N \models B P A$ or $N \models P A$, and $\ell=2,3$. We define $\mathbf{s}=\mathbf{s}_{\ell}[N, D]$. Omitting $D$ means $D=N$.

(0) $D$ is an initial segment of $N$ closed under addition. $D$ is nontrivial if it has a nonstandard member. We require that:

(a) If $\ell=2$, then $d \in D$ implies that $N \models(\forall x)\left(x^{d}\right.$ exists $)$.

(b) The case $\ell=3$ is covered in (2)(c).

(1) $\Delta=\Delta_{\mathbf{s}}$ is either:

(a) in the case of BPA, the set of all bounded formulas, or

(b) in the case of $P A$, the set of all formulas $\varphi(x, y, z)$ with $\ell(x)=\ell(y)=1$, which satisfy $1.1(1),(2),(4),(7)$.

(2) $\mathbf{a} \in \operatorname{Or}(\mathbf{s})$ when the data of $\left(X_{\mathbf{a}},<_{\mathbf{a}}\right)$ is internally isomorphid8 to some $\left(X_{\mathbf{a}}, \leq_{\mathbf{a}}, d_{\mathbf{a}}, \mathcal{T}_{\mathbf{a}}\right)$ which satisfies:

(a) the set of elements of $X_{\mathbf{a}}$ is a $\Delta$-definable bounded subset of $N$.

(b) $<_{\mathbf{a}}$ is a $\Delta$-definable linear order on $X_{\mathbf{a}}$

(c) $d_{\mathbf{a}} \in X_{\mathbf{a}}$, and $\left(\left\{d: d<d_{\mathbf{a}}\right\},<_{\mathbf{a}}\right)$ is an initial segment of $N$ with the usual order.

- If $\ell=2$, we require that $d_{\mathbf{a}} \in D$.

- If $\ell=3$, we require that $d_{\mathbf{a}} \in D$ and $\left|X_{\mathbf{a}}\right|^{d_{\mathbf{a}}}$ exists.

(d) $\mathcal{T}_{\mathbf{a}}$ is defined and satisfies the conditions from 1.1. and its defining formulas depend uniformly on the the formula defining $X_{\mathbf{a}}$.

(3) Write $\mathbf{s}^{+}[N, D]$ to indicate that we close the set of orders of $\mathbf{s}[N, D]$ under taking Cartesian products, where $d_{\mathbf{a} \times \mathbf{b}}$ is understood to be nontrivial if $d_{\mathbf{a}}, d_{\mathbf{b}}$ are, and that the order on at least one of the pairs is given by the Gödel pairing function.

${ }^{8}$ recall that as the set is listed with its order, this means: internally order-isomorphic. 
For use in later papers, we record here:

Definition 3.4. In the context of 3.3 , suppose $f$ is a nondecreasing function with $\operatorname{dom}(f)=N$ and range $(f) \subseteq\{I: I$ an initial segment of $N\}$, such that: $d \in f(a)$ implies that $a^{d}$ exists in $N$ and whenever a is nonstandard, $f(a)$ contains some nonstandard $d$. Define $\mathbf{s}[N, f]$ by requiring that for each $\mathbf{a}$,

(a) $d_{\mathbf{a}} \leq f\left(\max \left(X_{\mathbf{a}}\right)+1\right)$, and

(b) $f\left(\max \left(X_{\mathbf{a}}\right)+1\right)=I$ implies $d_{\mathbf{a}} \in I$.

Let us also give a name to a recurrent assumption.

Definition 3.5 (Reasonable models). We say $N \models B P A$ is reasonable when $a \in N$ implies that $\left\{a^{n}: n\right.$ a standard integer $\}$ is a bounded subset of $N$.

Claim 3.6. Let $N \models B P A$.

(1) If $N$ is $\aleph_{1}$-saturated or just recursively saturated, then $N$ is reasonable.

(2) $N$ is reasonable iff $N$ is a candidate in the sense of 4.2 below.

(3) If $D_{1}$ is a reasonable initial segment of $N$ then $D_{2}=\operatorname{cl}\left(D_{1}, N\right):=\{a \in N$ : for some $d \in D_{1}$ and standard $n, N=$ " $\left.a<d^{n} "\right\}$ is also reasonable.

(4) If $D$ is an initial segment of $N$ then $\operatorname{cl}(D, N)$ is closed under products.

Proof. (1),(2),(4) are immediate.

(3): Let $a_{2} \in D_{2}$ and $n \in N$ be standard. Let $d_{1} \in D_{2}$ and $m$ standard be such that $N \models d_{2}<\left(d_{1}\right)^{m}$. We define $x_{\ell}$ by induction on $\ell \leq n \cdot m$ as follows: $x_{0}=x, x_{\ell}=\left(x_{\ell}\right)^{d_{1}}$. Note that $\left(x_{\ell}\right)^{d_{\ell}}$ exists by the assumption on $D_{1}$. So $x_{n m}$ is well defined. Since

$$
x^{d_{2}} \leq x^{d_{1}{ }^{m}}=\left(x^{d_{1}}\right)^{d_{1}} \cdots\left(x^{d_{1}}\right)^{d_{1}} \leq x_{n m}
$$

where the $\operatorname{dots} \cdots$ indicate the multiplication has $m$ terms, $x^{d_{2}}$ exists.

Observation 3.7. If $N \models B P A$ and $\mathbf{s}=\mathbf{s}_{\ell}[N, D]$ for $\ell \in\{2,3\}$ then $\mathbf{s}$ is a hereditary weak $C S P$.

Claim 3.8. Let $N \models B P A$ and suppose that for $n, d \in N$ we have that $n^{d}$ exists. Then the tree of functions from $d$ to $n$ is definable in $N$, by bounded formulas. Specifically, the operations from 1.1.7) for the tree of sequences of length $d$ into $\left([0, n],<^{N}\right)$ are all definable by bounded formulas.

Proof. For compatibility with 1.1 , we denote $[0, n]$ by $X_{\mathbf{a}}$ and $d$ by $d_{\mathbf{a}}$. Let $\mathcal{T}$ denote the sequences of elements of $X_{\mathbf{a}}$ of length $\leq d_{\mathbf{a}}$. First we show that for each $\eta \in \mathcal{T}$ there is $c \in N$ which is a code for $\eta$ (and that this is uniformly and boundedly definable). This applies the long known fact that Gödel coding may be carried out in BPA; we sketch a proof for completeness, following the method of Wilkie-Paris [14.

We use $B$-adic coding (for $B=2$ ), representing each element of $N$ as a word in the finite alphabet $\{0,1\}$. (This ignores the empty word, which could be accommodated by using a different finite alphabet or $B=3$.) Since for each $\mathcal{T}_{\mathbf{a}}$ or $\mathcal{S}_{\mathbf{a}}$ we will be coding sequences of elements of uniformly bounded length, we don't need a 
separate symbol to indicate a transition between codes for distinct elements. Let $\operatorname{lgd}(x)$ denote $\max \left\{\ell: 2^{\ell} \leq x\right\}$, the dyadic length.

By $3.3(2)$, we may assume every element of $\mathrm{Or}(\mathbf{s})$ is isomorphic to a canonical one, i.e. to $\mathbf{a} \in \operatorname{Or}(\mathbf{s})$ where:

- $X_{\mathbf{a}}=\left[0, \max \left(X_{\mathbf{a}}\right)\right]$

- $\leq_{\mathbf{a}}$ agrees with the order of $N$ restricted to $X_{\mathbf{a}}$

- $d_{\mathbf{a}}$ is such that $\left(\max X_{\mathbf{a}}\right)^{d_{\mathbf{a}}}$ exists, which is equivalent to: $(\exists n)\left(\left|X_{\mathbf{a}}\right| \leq 2^{n} \leq 2\left|X_{\mathbf{a}}\right|\right) \wedge$ " $2^{n \cdot d_{\mathbf{a}}}$ exists".

- $(\exists n, m)\left(d_{\mathbf{a}} \leq 2^{m} \leq 2 d_{\mathbf{a}} \wedge\left|X_{\mathbf{a}}\right| \leq 2^{n} \leq 2\left|X_{\mathbf{a}}\right|\right.$ and $2^{n \cdot 2^{m}}$ exists $)$

- so w.l.o.g. $d_{\mathbf{a}}=2^{m},\left|X_{\mathbf{a}}\right|=2^{n}$.

For an element $a$ of $X_{\mathbf{a}}$, let $\operatorname{rep}_{B}(a)$ denote the $B$-adic representation of $a$ of length exactly $n+1$, padded with zeros if necessary (this is possible by the choice of $n$ ). In the expression below let " $2^{m}+\operatorname{rep}_{B}(a)$ " mean in base 2 , so this will effectively move $\operatorname{rep}_{B}(a)$ (which is a sequence of length $\leq n$ ) over $m$ spaces.

Let $\varphi_{\text {code }}(x, i, b)$ mean:

- $x<2^{n \cdot 2^{m+1}}$

- $i<d_{\mathbf{a}}$

- $b<\max X_{\mathbf{a}}$

- $\left(\exists x_{1} x_{2}\right)\left(x_{1} \wedge x_{2} \triangleleft x \wedge \operatorname{lgd}\left(x_{1}\right)=(n+1) i \wedge \operatorname{lgd}\left(x_{2}\right)=n+1 \wedge x_{2}=2^{n+1}+\operatorname{rep}_{B}(b)\right)$

Informally, $\varphi_{\text {code }}$ asserts that $x$ is the code for a sequence, thought of as consisting of no more than $d_{\mathbf{a}}$ consecutive blocks of length $n+1$ (leaving one extra space for the coding of $\mathcal{S}_{\mathbf{a}}$ in the proof of Claim 3.9 , the $(i+1)$ st of which is $\operatorname{rep}_{B}(b)$.

As written several values of $x$ may code the same sequence; we may avoid this by restricting to $x$ such that no $y<x$ codes the same sequence.

Then for any given $\mathbf{a} \in \operatorname{Or}(\mathbf{s})$, for our fixed values of $n, m$, we may naturally represent $\mathcal{T}_{\mathbf{a}}$ by

$$
\begin{aligned}
\mathcal{T}_{\mathbf{a}} & =\left\{x: x<2^{n \cdot 2^{m+1}},(\exists i<n)(\operatorname{lgd}(x)=(m+1) \cdot i)\right. \\
& \text { and } \left.(\forall j \leq i)\left(\exists x_{0}, x_{1}<x\right)\left(x_{0}{ }^{\frown} x_{1} \triangleleft x \text { and } 2^{m} \leq x_{0}<2^{m}+2^{n}\right)\right\}
\end{aligned}
$$

Since we have fixed the values of $X_{\mathbf{a}}$ (thus, of $\max \left(X_{\mathbf{a}}\right)$ ) and $d_{\mathbf{a}}$, we can easily build on $\psi_{\text {code }}$ to find bounded formulas defining: the partial order on elements of $\mathcal{T}$ by initial segment, length, concatenation, and value of the function at a given element of its domain.

Claim 3.9. Let $N \models B P A$ be reasonable and suppose that $\mathbf{s}=\mathbf{s}_{3}[N, D]$. Then $\mathbf{s}$ has simple exponentiation.

Proof. Recall that to say $\mathbf{s}$ has simple exponentiation means that for every a $\in$ $\operatorname{Or}(\mathbf{s})$ there is $\mathbf{b} \in \operatorname{Or}(\mathbf{s})$ such that $\left(X_{\mathbf{b}},<_{\mathbf{b}}\right)$ and $\left(\mathcal{S}_{\mathbf{a}},<_{\mathcal{S}_{\mathbf{a}}}\right)$ are internally isomorphic. Weak exponentiation adds that also $\left(Y_{\mathbf{b}},<_{\mathbf{b}}\right),\left(Y_{\mathbf{a}},<_{\mathbf{a}}\right)$ are internally isomorphic.

Let $\mathbf{a} \in \operatorname{Or}(\mathbf{s})$ be nontrivial. By construction of $\mathbf{s}_{3}$, we may identify $\mathbf{a}$ with its "canonical" isomorphic image, so assume $X_{\mathbf{a}}$ is an initial segment of $N$ with the usual order. We will show that the hypothesis that $\left|X_{\mathbf{a}}\right|^{d_{\mathbf{a}}}$ exists, i.e. that $\left(\max \left(X_{a}\right)\right)^{d_{\mathrm{a}}}$ exists, already ensures weak exponentiation. 
As $\mathbf{s}=\mathbf{s}_{3}(N)$, there is $\mathbf{b} \in \operatorname{Or}(\mathbf{s})$ so that $\left(X_{\mathbf{b}}, \leq_{\mathbf{b}}\right)$ is internally isomorphic to $\left(\left[0, n_{*}\right],<^{N}\right)$ and this isomorphism takes $d_{\mathbf{b}}$ to $d_{*}$ and $\mathcal{T}_{\mathbf{b}}$ to $\mathcal{T}_{*}$. Then $\mathcal{T}_{*}$ is boundedly definable by Claim 3.8. Continuing in the notation of that proof, to code the tree consisting of $\mathcal{S}_{\mathbf{a}}=\mathcal{T}_{\mathbf{a}} \times\{0,1\}$, define $f$ as follows:

$$
\begin{aligned}
& \text { if } x=\left(\sum_{i<\lg (x)}\left(b_{i}+2^{m}\right) \cdot 2^{(m+1) i}, \mathbf{i}\right), \mathbf{i} \in\{0,1\}, \\
& \text { then } f(x)=\sum_{i<\lg (x)}\left(b_{i}+2^{m}\right) \cdot 2^{(m+1) i}+\mathbf{i} .
\end{aligned}
$$

recalling that we have left one unit of space by arranging our coding into blocks of size $n+1$. Let $\psi_{\text {code }}^{+}=\operatorname{range}(f)$, which is definable by a bounded formula. If $x_{1}, x_{2} \in \mathcal{S}_{\mathbf{a}}$ then $x_{1}<\mathcal{S}_{\mathbf{a}} x_{2}$ iff $f\left(x_{1}\right)<f\left(x_{2}\right)$. This proves that $\left(\mathcal{S}_{\mathbf{a}},<_{\mathcal{S}_{\mathbf{a}}}\right)$ is order-isomorphic to an initial segment of $N$.

Let $n_{*}=$ max range $(f)$. Since $N$ is reasonable, there is some nonstandard $d_{*} \in$ $N$ such that $\left(n_{*}\right)^{d_{*}}$ exists. Applying Claim 3.8 once more, we have a tree $\mathcal{T}_{*}$ of functions from $d_{*}$ to $\left[0, n_{*}\right]$ which is definable by bounded formulas. By the definition of $\mathbf{s}_{3}(N)$, there is $\mathbf{b} \in \operatorname{Or}(\mathbf{s})$ with $X_{\mathbf{b}}$ internally order-isomorphic to $\left[0, n_{*}\right]$ under the usual order so that the image of $d_{\mathbf{b}}$ is $d_{*}$.

This proves $\mathbf{s}$ has simple exponentiation.

If in addition $\left(n_{*}\right)^{d_{\mathbf{a}}}$ exists, let $d_{\mathbf{b}}=d_{\mathbf{a}}$. Then the same internal order-isomorphism takes $Y_{\mathbf{b}}$ to $Y_{\mathbf{a}}$, and $\mathbf{s}$ has weak exponentiation. This completes the proof.

Claim 3.10. Suppose $N=B P A, N$ is reasonable and $\mathbf{s}=\mathbf{s}_{3}[N, D]$. Then $\mathbf{s}$ is a CSP.

Proof. As $\mathbf{s}$ is a hereditary weak c.s.p. there are two potentially missing conditions, $1.1(5)-(6)$.

Let nontrivial $\mathbf{a}, \mathbf{b}$ be given. By definition of $\mathbf{s}_{3}$, we may assume that $X_{\mathbf{a}}, X_{\mathbf{b}}$ are internally order- isomorphic to initial segments $\left[0, n_{\mathbf{a}}\right],\left[0, n_{\mathbf{b}}\right]$ of $N$ respectively (so in what follows we identify $X_{\mathbf{a}}, X_{\mathbf{b}}$ with these images). Without loss of generality, $n_{\mathbf{a}} \leq n_{\mathbf{b}}$. Let $\operatorname{Pr}: N \times N \rightarrow N$ denote the pairing function $(x, y) \mapsto(x+y+1)^{2}+x$. Consider the set $X_{\mathbf{a} \times \mathbf{b}}=\left\{\operatorname{Pr}(x, y): x \in X_{\mathbf{a}}, y \in X_{\mathbf{b}}\right\} \subseteq\left[0,\left(n_{\mathbf{a}}+n_{\mathbf{b}}+1\right)^{2}\right]$. Then $\operatorname{Pr}$ is an isomorphism from $X_{\mathbf{a}} \times X_{\mathbf{b}}$ onto an initial segment of $N$ which we call $X_{\mathbf{c}}$. Let $\leq_{\mathbf{c}}=\leq^{N}$ be the usual order. Let the order $\leq_{\mathbf{a} \times \mathbf{b}}$ be such that $\operatorname{Pr}$ is an orderisomorphism from $\left(X_{\mathbf{a} \times \mathbf{b}}, \leq_{\mathbf{a} \times \mathbf{b}}\right)$ onto $\left(X_{\mathbf{c}}, \leq_{\mathbf{c}}\right)$. Note that ${ }^{9}$ this pairing function satisfies $1.1(6)$.

Let $n_{*}=\max \left(X_{\mathbf{c}}\right)$. As we assumed $N$ is reasonable, there is some nonstandard $d_{*}$ such that $\left(n_{*}\right)^{d_{*}}$ exists. Let $d_{\mathbf{c}}=d_{*}$. Now existence of the tree $\mathcal{T}_{\mathbf{c}}$ is by Claim 3.8. Recalling the closure under isomorphism from $3.3(2)$, the product $\mathbf{a} \times \mathbf{b}$ is indeed a nontrivial element of $\mathbf{s}$. Thus $1.1(5)$ holds, which completes the proof.

Conclusion 3.11. Assume $N \models B P A, N$ is reasonable and $\mathbf{s}=\mathbf{s}_{3}(N, D)$. Then $\mathbf{s}$ is a cofinality spectrum problem with exponentiation, and so Theorem 2.10 applies: $\mathfrak{p}_{\mathrm{s}}=\mathfrak{t}_{\mathrm{s}}$.

\footnotetext{
${ }^{9} \mathrm{An}$ independent proof that $1.1(6)$ will be satisfied on some pair, assuming only that the c.s.p. is weak and that cardinality grows [which will be true in any such $N$ ] is given in the next section.
} 


\section{Saturated models of Peano arithmetic}

We now apply the above analysis to cuts in models of Peano arithmetic.

Theorem B. (Pabion, 1982 [13]) Let $\lambda>\aleph_{0}$ and $M$ be a model of PA. Then $M$ is $\lambda$-saturated iff $(M,<)$ is $\lambda$-saturated.

Theorem C. (Shelah, 1978 [16] Cor. 2.7 pps. 337-341)

(1) If $\mathcal{D}$ is a regular ultrafilter on $\lambda$ such that $M^{\lambda} / \mathcal{D}$ is $\lambda^{+}$-saturated for $M$ some model of linear order, then $N^{\lambda} / \mathcal{D}$ is 1-atomically- $\lambda^{+}$-saturated for any $N$ in a countable language, i.e. $\lambda^{+}$-saturated for types consisting of atomic formulas e.g. $x<a, b<x$.

(2) Hence if $T$ has the strict order property it is maximal in Keisler's order.

Pabion's theorem may be derived from the proof of Shelah's theorem just quoted. It was known that Keisler's order had a maximum class, so to show maximality of linear order, it sufficed to show that realizing types in the language of linear order (in some ultrapower) ensured realization of types in some given maximal theory, such as PA. The proof of Theorem C shows that in some language expanding linear order, the types of the given theory may be coded in such a way that omission of a type corresponds to a cut in a linear order; the point as regards Pabion's theorem is that this coding argument does not rely on ultrapowers except insofar as ultrapowers commute with reducts, and so may be carried out in any model of Peano arithmetic.

Observation 4.1. For $M$ a model of linear order $(<)$ and uncountable $\lambda, M$ is 1-atomically $\lambda$-saturated iff $\theta_{1}+\theta_{2} \geq \lambda$ whenever $M$ has a cut of cofinality $\left(\theta_{1}, \theta_{2}\right)$ and $\theta_{1}+\theta_{2} \geq \aleph_{0}$ (equivalently, $>2$, since we may have $a(1,1)$-cut). Usually, we can omit the "1-atomically," as e.g. in the order reduct of a model of PA or in $\operatorname{Th}(\mathbb{Q},<)$.

In Observation 4.1, note that if we use $x \leq y$, then $\min \left\{\theta_{1}, \theta_{2}\right\} \geq \aleph_{0}$ but then an $\left(\theta_{1}, 1\right)$-cut does not give incompactness.

The present results are an improvement in two respects. First, we can restrict to the case $\theta_{1}=\theta_{2}$, i.e. symmetric cuts. Second, our results are for bounded Peano arithmetic, not just PA.

Definition 4.2. Call $N$ a candidate model when $N$ is a model of bounded Peano arithmetic (with no last element) which is reasonable, i.e. such that for any $a \in N$ there is a nonstandard $d$ such that $a^{d}$ exists. We call $\mathbf{s}$ a candidate c.s.p. when $\mathbf{s}=\mathbf{s}_{3}[N]$ for a reasonable $N$.

To connect to saturation, we bring in a definition from 8 .

Definition 4.3. (8] Definition 3.41) Let $\mathbf{s}$ be a cofinality spectrum problem and $\lambda$ a regular cardinal. Let $p=p\left(x_{0}, \ldots x_{n-1}\right)$ be a consistent partial type with parameters in $M_{1}^{+}$. We say that $p$ is a Or-type over $M_{1}^{+}$if: $p$ is a consistent partial type in $M_{1}^{+}$and for some $\mathbf{a}_{0}, \ldots \mathbf{a}_{n-1} \in \operatorname{Or}(\mathbf{s})$, we have that

$$
p \vdash \bigwedge_{i<n} " x_{i} \in X_{\mathbf{a}_{i}} "
$$


and $p$ is finitely satisfiable in $X_{\mathbf{a}_{0}} \times \cdots \times X_{\mathbf{a}_{n-1}}$. We say simply that $M_{1}^{+}$is $\lambda$ Or-saturated if every Or-type over $M_{1}^{+}$over a set of size $<\lambda$ is realized in $M_{1}^{+}$. Finally, we say that $\mathbf{s}$ is $\lambda$-Or-saturated if $M_{1}^{+}$is.

Claim 4.4. (8] Claim 3.43) Let $\mathrm{s}$ be a cofinality spectrum problem. If $\kappa<$ $\min \left\{\mathfrak{p}_{\mathbf{s}}, \mathfrak{t}_{\mathbf{s}}\right\}$ then $\mathbf{s}$ is $\kappa^{+}$-Or-saturated.

Remark 4.5. Since by our definition any $\mathbf{a} \in \operatorname{Or}(\mathbf{s})$ has a maximum element, Or-saturation does not a priori guarantee that the cofinality of the model is large.

Observation 4.6. For a model $N$ of $P A$, the following are equivalent:

(1) $N$ is $\lambda$-saturated.

(2) $\operatorname{cf}(N) \geq \lambda$ and $N$ is boundedly $\lambda$-saturated, that is, $N i \leq a$ is $\lambda$-saturated for every $a \in N$.

Proof. It suffices to prove (2) implies (1). Given a type $p(x)$ of cardinality $<\lambda$, write $p(x)=\left\{\varphi_{i}\left(x, \bar{a}_{i}\right): i<\lambda\right\}$. Since $\operatorname{cf}(N) \geq \lambda$ there is some $a_{*} \in N$ such that $p(x) \cup\left\{x<a_{*}\right\}$ is finitely satisfiable. Let $b_{*}=2^{a_{*}}$. Now for each $i$ there are $c_{i}<b_{*}$ and $\varphi_{i}^{\prime}\left(x, y, b_{*}\right)$ which is $b_{*}$-bounded [meaning that all quantifiers are of the form $\left(\exists z<b_{*}\right)$ or $\left.\left(\forall z<b_{*}\right)\right]$ and $N \models(\forall x)\left(\varphi_{i}\left(x, \bar{a}_{i}\right) \equiv \varphi_{i}^{\prime}\left(x, c_{i}, b_{*}\right)\right)$. Let $p^{\prime \prime}(x)=\left\{\varphi_{i}^{\prime}\left(x, c_{i}, b_{*}\right) \wedge x<a_{*}: i<\lambda\right\}$. This is a finitely satisfiable type in $N i_{\leq b_{*}}$. Its realization implies realization of $p$, and it is realized by hypothesis (2).

Theorem 4.7. Let $N$ be a model of Peano arithmetic and $\lambda$ an uncountable cardinal. If the reduct of $N$ to the language of order has cofinality $\geq \lambda$ and no $(\kappa, \kappa)$-cuts for $\kappa<\lambda$, then $N$ is $\lambda$-saturated.

Proof. We may assume $N$ is a nonstandard model. Hence it follows that $N$ is reasonable in the sense of 3.3 , i.e. $a \in N$ implies that $\left\{a^{n}: n\right.$ a standard integer $\}$ is a bounded subset of $N$. (If not, $\operatorname{cf}(N)<\lambda$.)

Assume $\operatorname{cf}(N) \geq \lambda$. Let $\mathbf{s}=\mathbf{s}_{3}(N)$ be from 3.3. By Conclusion 3.11, $\mathbf{s}$ is a csp with exponentiation. Thus Theorem 2.10 applies and $\mathfrak{p}_{\mathbf{s}}=\mathfrak{t}_{\mathbf{s}}$. By Claim 4.4 and Observation 4.6, $\mathbf{s}$ is $\min \left\{\mathfrak{p}_{\mathbf{s}}, \mathfrak{t}_{\mathbf{s}}, \operatorname{cf}(N,<)\right\}$-saturated. If $\mathfrak{t}_{\mathbf{s}} \geq \lambda$, we finish, so assume that $\mathfrak{t}_{\mathbf{s}}<\lambda$. By Theorem 2.10, there is $\mathbf{a} \in \operatorname{Or}(\mathbf{s})$ whose $X_{\mathbf{a}}$ contains a $\left(\mathfrak{t}_{\mathbf{s}}, \boldsymbol{t}_{\mathbf{s}}\right)$-cut. Recall from Definition 3.3 that since $\mathbf{s}=\mathbf{s}_{3}(N)$, for each $\mathbf{a} \in \operatorname{Or}(\mathbf{s}),\left(X_{\mathbf{a}}, \leq_{\mathbf{a}}\right)$ is internally order-isomorphic to an initial segment of $N$ with the usual order. Then $N$ has a $\left(\mathfrak{t}_{\mathbf{s}}, \mathfrak{t}_{\mathbf{s}}\right)$-cut, which completes the proof.

Theorem 4.8. Let $N$ be a model of BPA which is reasonable, i.e. for every $a \in N$ the set $\left\{a^{n}: n \in N\right.$ finite $\}$ is bounded. Then the following are equivalent:

(1) for every $n_{*} \in N$, the model $N_{<n_{*}}=N\left\lceil\left\{a: N \models a<n_{*}\right\}\right.$ is $\lambda$-saturated.

(2) for every $n_{*} \in N$, the model $N_{\leq n_{*}}$ considered as a linear order has no $(\kappa, \kappa)$-cuts for $\kappa=\operatorname{cf}(\kappa)<\lambda$.

Proof. (1) implies (2) is obvious, so assume (2) holds. Let $\mathbf{s}=\mathbf{s}_{3}(N)$ be from 3.3 . Then by $3.11 \mathrm{~s}$ is a cofinality spectrum problem with exponentiation, and $\mathfrak{p}_{\mathbf{s}}=\mathfrak{t}_{\mathrm{s}}$.

Let $\kappa=\mathfrak{p}_{\mathbf{s}}=\mathfrak{t}_{\mathbf{s}}$, so $\kappa$ is regular. By 2.9, some $\mathbf{a} \in \operatorname{Or}(\mathbf{s})$ has a $(\kappa, \kappa)$-cut. By the definition of $\mathbf{s}_{3}(N)$, any $X_{\mathbf{a}}$ is internally order-isomorphic to a bounded initial 
segment of $N$ with the usual order. Thus, some bounded initial segment of $N$ has a $(\kappa, \kappa)$-cut. As we've assumed (2), it must be that $\lambda \leq \kappa=\mathfrak{p}_{\mathbf{s}}=\mathfrak{t}_{\mathbf{s}}$.

Let $n_{*} \in N$ be given. As we assumed $N$ is reasonable, there is a nonstandard $d_{*}$ such that $N=" n_{*}{ }^{d_{*}}$ exists". Recalling $3.3(2)$, there is a nontrivial a $\in \operatorname{Or}(\mathbf{s})$ such that $\left(X_{\mathbf{a}}, \leq_{\mathbf{a}}\right)$ isomorphic to an initial segment of $N$, containing $\left[0, n_{*}\right]$, with the usual order. Thus, to prove (1), it will suffice to show that every $\mathbf{a} \in \operatorname{Or}(\mathbf{s})$ is $\gamma^{+}$Or-saturated for every $\gamma<\lambda$. By 4.4 , if $\gamma<\min \left\{\mathfrak{p}_{\mathbf{s}}, \mathfrak{t}_{\mathbf{s}}\right\}$ then $\mathbf{s}$ is $\gamma^{+}$-Or-saturated. Since $\lambda \leq \mathfrak{p}_{\mathbf{s}}=\mathfrak{t}_{\mathbf{s}}$, this completes the proof.

Note that Theorems 4.7 and 4.8 show that the situation in models of Peano arithmetic is very different from that in real closed fields, as shown by the next quoted theorem. By "asymmetric cut" we mean a cut in which the infinite cofinalities of each side are not equal.

Theorem D (Theorem 1.1 of Shelah [21]). Let $K$ be an arbitrary ordered field. Then there is a symmetrically complet $\emptyset^{10}$ real closed field $K^{+}$containing $K$ such that any asymmetric cut of $K$ is not filled. So if $K$ is not embeddable into $\mathbb{R}$, then $K^{+}$and $K$ necessarily have an asymmetric cut.

\section{ON THE LOCAL CARDINALS $\mathfrak{p}_{\mathbf{s}, \mathbf{a}}$ AND $\mathfrak{t}_{\mathbf{s}, \mathbf{a}}$}

Returning to CSPs generally, in this section we prove Theorem 5.3, a complementary result to Theorem 2.10. The theorem shows that the local cardinals $\mathfrak{p}_{\mathbf{s}, \mathbf{a}}$ and $\mathfrak{t}_{\mathbf{s}, \mathbf{a}}$ from Definition 5.1 need not normally agree, even in CSPs arising from models of Peano arithmetic, if the underlying $M_{1}^{+}$is not uniformly saturated.

Definition 5.1. Let $\mathbf{s}$ be a CSP or weak CSP, and $\mathbf{a} \in \operatorname{Or}(\mathbf{s})$.

(1) Let $\mathfrak{p}_{\mathbf{s}, \mathbf{a}}$ be $\min \left\{\kappa\right.$ : there are regular $\kappa_{1}, \kappa_{2}$ such that $\kappa_{1}+\kappa_{2}=\kappa$ and $X_{\mathbf{a}}$ has $a\left(\kappa_{1}, \kappa_{2}\right)$-cut $\}$.

(2) Let $\mathfrak{t}_{\mathbf{s}, \mathbf{a}}$ be $\min \left\{\kappa: \kappa \geq \aleph_{0}\right.$ and there is in the tree $\mathcal{T}_{\mathbf{a}}$ a strictly increasing sequence of cofinality $\kappa$ with no upper bound $\}$.

We need a preliminary lemma.

Lemma 5.2. Let $\mathbf{s}$ be a cofinality spectrum problem, $\mathbf{a} \in \operatorname{Or}(\mathbf{s})$. Suppose $\left(\mathcal{S}_{\mathbf{s}},<_{\mathcal{S}_{\mathbf{s}}}\right)$ has a $(\kappa, \kappa)$-cut, for $\kappa \leq \mathfrak{t}_{\mathbf{s}}$. Then either $X_{\mathbf{a}}$ has an $(\kappa, \kappa)$-cut or else $\mathcal{T}_{\mathbf{a}}$ has a branch of cofinality $\kappa$ with no upper bound.

Proof. Let $(\bar{a}, \bar{b})=\left(\left\langle\left(a_{i}, t_{i}\right): i<\kappa\right\rangle,\left\langle\left(b_{i}, s_{i}\right): i<\kappa\right\rangle\right)$ witness the cut in $\mathcal{S}_{\mathbf{a}}$, with $a_{i}, b_{i} \in \mathcal{T}_{\mathbf{a}}$ and $t_{i}, s_{i} \in\left\{0_{\mathbf{a}}, 1_{\mathbf{a}}\right\}$ for each $i<\kappa$. By definition of cut, we may assume $\kappa$ is regular.

Step 1: Simplifying the presentation of intervals. By the pigeonhole principle, we may assume the sequences $\left\langle t_{i}: i<\kappa\right\rangle$ and $\left\langle s_{i}: i<\kappa\right\rangle$ are constant. For each $i<\kappa$, write $A_{i}$ for the closed interval in the linear order $S_{\mathbf{a}}$ whose endpoints are given by

\footnotetext{
${ }^{10}$ This means that any decreasing sequences of closed bounded intervals, of any ordinal length, has nonempty intersection.
} 
$\left(a_{i}, t_{i}\right)$ and $\left(a_{i},\left|t_{i}-1\right|\right)$, and likewise for $B_{i}$. By the construction of $S_{\mathbf{a}}$, any two intervals of this form are either concentric or disjoint.

Let $\bar{A}=\left\langle A_{i}: i<\kappa\right\rangle$ and $\bar{B}=\left\langle B_{i}: i<\kappa\right\rangle$.

The task of this step will be to prove that without loss of generality, $\bar{A}$ consists of either pairwise concentric or pairwise disjoint intervals, and likewise for $\bar{B}$. Here "concentric" means either either concentric decreasing: $j<i<\kappa \Longrightarrow C_{j} \supsetneq C_{i}$ [for $C=A$ or $B$ ] or concentric increasing: $j<i<\kappa \Longrightarrow C_{i} \supsetneq C_{j}$.

If $\kappa=\aleph_{0}$, then by Ramsey's theorem ${ }^{11}$ we may assume that $\bar{A}$ consists either of concentric intervals or disjoint intervals moving right, meaning $j<i<\kappa$ implies $A_{j} \cap A_{i}=\emptyset$ and $\left(\forall x \in A_{j}\right)\left(\forall y \in A_{i}\right)(x<y)$. Likewise, we may assume that $\bar{B}$ consists either of concentric intervals or of disjoint intervals moving left, meaning $j<i<\kappa$ implies $B_{j} \cap B_{i}=\emptyset$ and $\left(\forall x \in B_{j}\right)\left(\forall y \in B_{i}\right)(y<x)$.

If $\kappa>\aleph_{0}$, let $a \wedge b$ denote the maximal common initial segment of $a, b \in \mathcal{T}_{\mathbf{a}}$. For this argument, we use $c$ to denote either $a$ or $b$. For each $i$, the sequence $\left\langle\lg _{\mathbf{a}}\left(c_{i} \wedge c_{j}\right): j \in[i, \kappa)\right\rangle$ is a sequence of elements of $Y_{\mathbf{a}} \subseteq X_{\mathbf{a}}$ [recall Definition 1.7 bounded by $\lg _{\mathbf{a}}\left(c_{i}\right)$. Thus, for some club $E_{i}$ of $\kappa$ with $\min E_{i}>i$, we have that $\bar{\ell}_{i}:=\left\langle\lg _{\mathbf{a}}\left(c_{i} \wedge c_{j}\right): j \in E_{i}\right\rangle$ is either constant or $<_{\mathbf{a}}$-decreasing. Let $S \subseteq \kappa$ be a stationary set of $i$ on which we get the same outcome (either always constant or always decreasing).

Let $E=\bigcap\left\{\epsilon<\kappa: \epsilon\right.$ a limit ordinal and $\left.\epsilon \in \bigcap_{i<\epsilon} E_{i}\right\}$, so $E$ is a club of $\kappa$. There are several cases:

(1) First case: for all $i \in S, \bar{\ell}_{i}$ is constant.

(a) If $i \in S, j \in E_{i}$ implies $c_{i} \wedge c_{j}=c_{i}$, then $\left\langle c_{i}: i \in S \cap E\right\rangle$ is a $\triangleleft \mathcal{T}_{\mathrm{a}}$-increasing sequence so we are in the concentric decreasing case.

(b) If $i \in S, j \in E_{i}$ implies $\lg _{\mathbf{a}}\left(c_{i} \wedge c_{j}\right)<\lg \left(c_{i}\right)$ then by Fodor's lemma there is $\gamma \in \kappa$ and a stationary subset $X$ of $\kappa$ such that $i \in X$ and $j \in E_{i}$ implies $\lg _{\mathbf{a}}\left(c_{i} \wedge c_{j}\right)=\gamma$, so we are in the pairwise disjoint case.

(2) Second case: for all $i \in S, \bar{\ell}_{i}$ is $<_{\mathbf{a}}$-decreasing. (Remember that the branches of $\mathcal{T}_{\mathbf{a}}$ are internally pseudofinite, but not necessarily well-ordered from an external point of view.) Let $X=S \cap E$. Then $\left\langle c_{i}: i \in X\right\rangle$ is a $\triangleleft_{\mathcal{T}_{\mathbf{a}}}$-decreasing sequence, so we are in the concentric increasing case.

Step 2: The concentric cases. Suppose both $\bar{A}$ and $\bar{B}$ are concentric. Then $\left\langle a_{i}\right.$ : $i\langle\kappa\rangle$ and $\left\langle b_{i}: i\langle\kappa\rangle\right.$ are both $\triangleleft_{\mathbf{a}}$-linearly ordered sequences in $\mathcal{T}_{\mathbf{a}}$. There are four cases depending on whether each of these sequences is $\triangleleft_{\mathbf{a}}$-increasing or decreasing.

(a) Suppose both $\left\langle a_{i}: i\langle\kappa\rangle\right.$ and $\left\langle b_{i}: i\langle\kappa\rangle\right.$ are $\triangleleft_{\mathbf{a}}$-increasing. If they lie along eventually different branches, the original sequence $(\bar{a}, \bar{b})$ could describe only a pre-cut and not a cut according to the definition of $\mathcal{S}_{\mathbf{a}}$, so we get a contradiction. If they lie along the same branch, then it must be that $\mathcal{T}_{\mathbf{a}}$ has a branch of cofinality $\kappa$, as desired.

\footnotetext{
${ }^{11}$ This also holds if $\kappa$ is weakly compact.
} 
(b) If $\left\langle a_{i}: i\langle\kappa\rangle\right.$ is $\triangleleft_{\mathbf{a}}$-increasing while $\left\langle b_{i}: i<\kappa\right\rangle$ is $\triangleleft_{\mathbf{a}}$-decreasing, these and form a cut $(*)$ in the linearly ordered set

$$
\left(\left\{c \in \mathcal{T}_{\mathbf{a}}: c \triangleleft b_{0}\right\}, \triangleleft\right)
$$

Then the projections $\left(\left\langle\lg \left(a_{i}\right): i<\kappa\right\rangle,\left\langle\lg \left(b_{j}\right): j<\kappa\right\rangle\right.$ form a pre-cut $(* *)$ in $X_{\mathbf{a}}$. If this pre-cut $(* *)$ were realized, say by $t$, then $b_{0} \uparrow t$ realizes the cut $(*)$, contradiction. This shows that $X_{\mathbf{a}}$ has a $(\kappa, \kappa)$-cut.

(c) If $\left\langle a_{i}: i<\kappa\right\rangle$ and $\left\langle b_{i}: i<\kappa\right\rangle$ are both $\triangleleft_{\mathcal{T}_{\mathbf{a}}}$-decreasing, the original sequence $(\bar{a}, \bar{b})$ will not describe a cut, so we ignore this case.

(d) If $\left\langle b_{i}: i\langle\kappa\rangle\right.$ is $\triangleleft_{\mathcal{T}_{\mathbf{a}}}$-increasing while $\left\langle a_{i}: i\langle\kappa\rangle\right.$ is $\triangleleft_{\mathcal{T}_{\mathbf{a}}}$-decreasing, the argument is parallel to case (b).

Step 3: Not both concentric. Again, there are several possibilities.

Suppose first that neither $\bar{A}$ nor $\bar{B}$ is concentric, so $\bar{A}$ is a sequence of disjoint intervals moving right and $\bar{B}$ is a sequence of disjoint intervals moving left.

Consider the sequence $\left\langle c_{i}: i\langle\kappa\rangle\right.$ where $c_{i}:=\operatorname{lub}\left\{a_{i}, b_{i}\right\}$ in the tree $\mathcal{T}_{\mathbf{a}}$. By definition of $\bar{a}$ and $\bar{b}$, this sequence will be either eventually constant or a path through the tree $\mathcal{T}_{\mathbf{a}}$.

If the sequence is a path through the tree, then $\left\langle\left(c_{i}, 0\right): i<\kappa\right\rangle$ is cofinal in $\bar{a}$ and $\left\langle\left(c_{i}, 1\right): i<\kappa\right\rangle$ is cofinal in $\bar{b}$. So the path $\left\langle c_{i}: i<\kappa\right\rangle$ cannot have an upper bound, as given any such upper bound $d_{*}$, by definition of $\left(\mathcal{S}_{\mathbf{a}},<\mathcal{S}_{\mathbf{a}}\right)$, we would have that $\left(d_{*}, 0\right)$ and $\left(d_{*}, 1\right)$ both realize the original cut, contradiction.

So in this case, there is a path through $\mathcal{T}_{\mathbf{a}}$ of length $\kappa$ with no upper bound.

If the sequence is eventually constant, then there is $i_{*}<\kappa$ such that all $\left\{a_{i}, b_{i}\right.$ : $\left.i_{*}<i<\kappa\right\}$ are immediate successors of the same node, say $a_{*}$, in $\mathcal{T}_{\mathbf{a}}$. $\operatorname{So} \lg \left(a_{*}\right)<$ $d_{\mathbf{a}}$. By definition of the order $\left(\mathcal{S}_{\mathbf{a}},<_{\mathcal{S}_{\mathbf{a}}}\right)$ and the case we are in, this means

$$
\left(\left\langle a_{i}\left(\lg \left(a_{i}\right)-1\right): i_{*}<i<\kappa\right\rangle,\left\langle b_{i}\left(\lg \left(b_{i}\right)-1\right): i_{*}<i<\kappa\right\rangle\right)
$$

is a pre-cut in $X_{\mathbf{a}}$. Suppose for a contradiction it were realized by $x$; then $c_{*}:=$ $a_{*} \Upsilon\langle x\rangle$ would exist since $\lg \left(a_{*}\right)<d_{\mathbf{a}}$. Then in $\left(\mathcal{S}_{\mathbf{a}},<_{\mathcal{S}_{\mathbf{a}}}\right),\left(c_{*}, 0\right)$ and $\left(c_{*}, 1\right)$ would both realize the original cut $(\bar{a}, \bar{b})$, contradiction.

So in this case, $X_{\mathbf{a}}$ has a $(\kappa, \kappa)$-cut.

Otherwise, precisely one of $\bar{A}, \bar{B}$ is not concentric. The cases are parallel, so assume the non-concentric side is $\bar{B}$. Define $d_{i}$ for $i<\kappa$ by $d_{i}:=\operatorname{lub}\left\{b_{i}, b_{0}\right\}$ in the tree $\mathcal{T}_{\mathbf{a}}$. Writing $C_{i}$ for the interval $\left(\left(c_{i}, 0\right),\left(c_{i}, 1\right)\right)$ and $D_{i}$ for the interval $\left(\left(d_{i}, 0\right),\left(d_{i}, 1\right)\right)$ in $\mathcal{S}_{\mathbf{a}}$, we have that $\left\langle C_{i}: i<\kappa\right\rangle$ and both $\left\langle D_{i}: i<\kappa\right\rangle$ are concentric sequences of intervals with $D_{i} \subseteq C_{j}$ for all $i>>j$.

This reduces the problem to Step 2.

Step 4: Finish. We have shown that in each case either $X_{\mathbf{a}}$ has a $(\kappa, \kappa)$-cut or else $\mathcal{T}_{\mathbf{a}}$ has a strictly increasing path of length $\kappa$ with no upper bound, so this completes the proof. 
Theorem 5.3. Let $\kappa$ be a regular uncountable cardinal.

(1) Suppose we are given $M$ a model of $P A$ which is $\kappa$-saturated, and $a_{*} \in M$ nonstandard. Then we can find a countable set $X \subseteq M$ such that letting $N$ be the Skolem hull of $\left\{a \in M: M \models a \leq a_{*}\right\} \cup X$, we have that the reduct $(N,<)$ to the language of order has an $\left(\aleph_{0}, \aleph_{0}\right)$-cut.

(2) There is a cofinality spectrum problem $\mathbf{s}$ with $M_{1}^{\mathbf{s}}=N$ and $\mathbf{a} \in \operatorname{Or}(\mathbf{s})$ such that

$$
\mathfrak{t}_{\mathbf{s}, \mathbf{a}}<\mathfrak{p}_{\mathbf{s}, \mathbf{a}}
$$

in fact, $\mathfrak{t}_{\mathbf{s}, \mathbf{a}}=\aleph_{0}$ while $\mathfrak{p}_{\mathbf{s}, \mathbf{a}} \geq \kappa$.

(3) If in the conditions above, we have just that $M \models B P A$ and for some $r_{*}<a_{*}$ nonstandard $M=" a_{*}{ }^{r_{*}}$ exists", this is enough.

Proof. First we prove (1). Let $A=M \uparrow a_{*}$.

Let $\left\langle F_{n}: n<\omega\right\rangle$ list the Skolem functions of $M$, each appearing infinitely often (for transparency). Let $k_{n}$ be the arity of $F_{n}$, and without loss of generality $k_{n} \leq n$.

Let $p$ be the type in the variables $z, x_{i}(i<\omega), y_{i}(i<\omega)$ and parameter $a_{*}$ expressing:

(a) $z<a_{*}$ is nonstandard

(b) $x_{0}=a_{*}$

(c) $y_{0}=\left(a_{*}\right)^{z}$

(d) $m<n<\omega$ implies $a_{*}=x_{0} \leq x_{m}<x_{n}<y_{n}<y_{m} \leq y_{0}$

(e) letting $B_{n}=\left\{F_{n}\left(e_{0}, \ldots, e_{k_{n}-1}\right): e_{\ell} \in A \cup\left\{x_{\ell}, y_{\ell}: \ell<n\right\}\right\}$, we have that $B_{n} \cap\left(x_{n}, y_{n}\right)=\emptyset$.

Let us check that $p$ is consistent. By the $\kappa$-saturation of $M$, this will suffice to show it is realized.

Fix $n<\omega$ and consider a finite fragment of $p\left\lceil z, x_{0}, \ldots, x_{n}, y_{0}, \ldots, y_{n}\right.$. Let $\ell$ be the maximal exponent appearing in conditions of the form $(e)$. We are looking for $r, a_{0}, \ldots, a_{n}, b_{0}, \ldots, b_{n}$ such that

- $r$ is nonstandard, or simply above some given natural number

- $a_{*}=a_{0}<\cdots<a_{n}<b_{n}<\cdots<b_{0}=\left(a_{*}\right)^{r}$

- $m \leq n$ implies $B_{m} \cap\left(a_{m}, b_{m}\right)=\emptyset$

For each $n$, the set $B_{n}$ is definable in $M$ (since it only involves one function $F_{n}$, of arity $\left.k_{n}\right)$ and of power $c_{n}$, where $c_{n} \approx(|A|+2 n)^{k_{n}}<a_{*}{ }^{k_{n}+1}$. Let $B_{n}\left(w, v_{0}, \ldots, v_{n}, v_{0}^{\prime}, \ldots, v_{n}^{\prime}\right)$ denote the set

$$
\left\{F_{n}\left(e_{0}, \ldots, e_{k_{n}-1}\right): e_{\ell} \leq w \vee e_{\ell} \in\left\{v_{0}, \ldots, v_{n}, v_{0}^{\prime}, \ldots, v_{n}^{\prime}\right\}\right\}
$$

which will likewise have size $\leq w^{k_{n}} \cdot(2 n+2)^{k_{n}}<w^{k_{n}+1}$. Let $\varphi_{n}\left(w, v_{0}, \ldots, v_{n}, v_{0}^{\prime}, \ldots, v_{n}^{\prime}\right)$ assert that

$$
(\forall t)\left(\exists s \leq x^{k_{n}+1} \cdot t\right)(\exists a \exists b)\left(a<b \leq s \wedge b-a>t \wedge(a, b) \cap B_{n}(x)=\emptyset\right)
$$

Then clearly for all $m \leq n, M \models \forall w \forall \bar{v} \forall \bar{v}^{\prime} \varphi_{m}\left(w, \bar{v}, \bar{v}^{\prime}\right)$, recalling that $k_{n}$ is fixed so the exponential notation abbreviates multiplication. 
Choosing $r$ such that $\left(k_{0}+2\right)\left(k_{1}+2\right) \cdots\left(k_{n}+2\right) \leq r<a_{*}$ and $b_{*}=b_{0}=\left(a_{*}\right)^{r}$ we may then choose $a_{1}, b_{1}, \ldots, a_{n}, b_{n}$ by induction on $\ell \leq n$ such that:

- $k<\ell \Longrightarrow a_{k}<a_{\ell}<b_{\ell}<b_{k}$

- $\left(a_{0}, b_{0}\right)=\left(a_{*},\left(a_{*}\right)^{r}\right)$

- $b_{\ell}-a_{\ell}>\left(a_{*}\right)^{n \cdot(n+1-\ell)}$

For $\ell+1$, we have $b_{\ell}-a_{\ell}>\left(a_{*}\right)^{(n+2)(n+1-\ell)}$ and $B\left(a_{*}, a_{0}, \ldots, a_{\ell}, b_{0}, \ldots, b_{\ell}\right)$ is internally a set with $\leq\left(a_{*}\right)^{k_{\ell}+1} \leq\left(a_{*}\right)^{n+1}<\left(a_{*}\right)^{n+2}$ elements, so there is room.

This completes the verification that $p$ is consistent, therefore (by saturation) realized. For the remainder of the proof, fix realizations $r, a_{i}(i<\omega), b_{i}(i<\omega)$ of the type $p$.

[With a little more care, using $\left(a_{*}\right)^{d_{*} \cdot\left(d_{*}+1-\ell\right)}$ for $\ell<\omega$, we could alternately have chosen the entire countable sequence by induction, avoiding the appeal to the type and $\aleph_{1}$-saturation.]

Let $N$ be the Skolem hull of $M \uparrow a_{*} \cup\left\{a_{n}, b_{n}: n<\omega\right\}$, so $N \models P A$. Let $\mathbf{s}=\mathbf{s}_{1}^{+}[N]$ be the canonical CSP from Definition 3.3. Then:

(a) $N \preceq M$.

(b) $M_{\leq a_{*}}=N_{\leq a_{*}}$ so $\left(N,<^{N}\right)$ is saturated below $a_{*}$ by definition of $M$.

(c) $\left(\left\langle a_{n}: n<\omega\right\rangle,\left\langle b_{n}: n<\omega\right\rangle\right)$ is an $\left(\aleph_{0}, \aleph_{0}\right)$-cut, because by construction the Skolem functions do not fill it.

(d) Thus, if a is such that $X_{\mathbf{a}}=M_{\leq a_{*}}=N_{\leq a_{*}}$, and, say, $d_{\mathbf{a}}=\max X_{\mathbf{a}}$, then $\mathfrak{p}_{\mathbf{s}, \mathbf{a}} \geq \kappa$ whereas if $\mathbf{b}$ is such that $X_{\mathbf{b}}=N_{\leq b_{*}}$, and, say, $d_{\mathbf{b}}=\max X_{\mathbf{b}}$, then $\mathfrak{p}_{\mathbf{s}, \mathbf{b}}=\aleph_{0}$.

This completes the proof of (1). We continue the argument to prove (2). (3) will follow from the proof.

Let a be such that $X_{\mathbf{a}}=N_{\leq a_{*}}$. Recalling the small nonstandard exponent $r$, let $d_{*}=r+1$ and consider the definable subtree $\mathcal{T} \subseteq \mathcal{T}_{\mathbf{a}}$ consisting of sequences of length $<d_{*}$ of numbers $<a_{*}$. $\mathcal{T}$ has cardinality $a_{*}^{r}$ [the tree $\mathcal{T}_{\mathbf{a}}$ will be at least as large].

Recalling that $N \models P A, \mathrm{~s}$ is closed under strong exponentiation, so there is an injection from $\mathcal{S}_{\mathbf{a}}$ into some $X_{\mathbf{a}}$. Composing with the Gödel pairing function if needed, we may assume there is a definable injection of $\mathcal{S}_{\mathbf{a}}$ (as a set) into some $X_{\mathbf{b}} \subseteq$ $N$. Applying Conclusion 7.4, we obtain a definable order-isomorphism between $\left(\mathcal{S}_{\mathbf{a}},<_{\mathcal{S}_{\mathbf{a}}}\right)$ onto an initial segment of $M_{1}^{+}=N$. Since $\mathcal{T}$ is the set of all sequences from $X_{\mathbf{a}}$ to itself of length $\leq d_{\mathbf{a}}=a_{*}>>r$, the cardinality of $\mathcal{T}$ is $\geq a_{*}{ }^{r}=b_{*}$, so its image must contain the $\left(\aleph_{0}, \aleph_{0}\right)$-cut. Because $X_{\mathbf{b}}$ has an $\left(\aleph_{0}, \aleph_{0}\right)$-cut, necessarily $\left(\mathcal{S},<_{\mathcal{S}}\right)$ has such a cut.

By Lemma 5.2 either $X_{\mathbf{a}}$ has an $\left(\aleph_{0}, \aleph_{0}\right)$-cut or else $\mathcal{T}_{\mathbf{a}}$ has a branch of cofinality $\aleph_{0}$ with no upper bound. Since $X_{\mathbf{a}}$ is $\kappa$-saturated by assumption, we must be in the second case. This shows that $\mathfrak{t}_{\mathbf{s}, \mathbf{a}}=\aleph_{0}$. On the other hand, $\mathfrak{p}_{\mathbf{s}, \mathbf{a}} \geq \kappa$, by the hypothesis of saturation.

Thus, the local cardinals $\mathfrak{p}_{\mathbf{s}, \mathbf{a}}$ and $\mathfrak{t}_{\mathbf{s}, \mathbf{a}}$ need not be equal, even in well behaved cofinality spectrum problems, if the model $M_{1}^{+}$is not uniformly saturated. 


\section{Characterizing The $\triangleleft^{*}$-MAXimal Class}

In this section we give the first real evidence that $\mathrm{SOP}_{2}$ is a dividing line by proving that, under instances of $\mathrm{GCH}, \mathrm{SOP}_{2}$ characterizes maximality in the interpretability order $\triangleleft^{*}$ which will be defined below. The proof uses cofinality spectrum problems. This answers an open question and also gives evidence for a recent conjecture, as we now explain. The use of GCH comes only from Fact 6.2 below. Recall:

Definition 6.1. ( $\mathrm{SOP}_{2}$, c.f. [24] 1.5) $\mathrm{T}$ has $\mathrm{SOP}_{2}$ if there is a formula $\varphi(\bar{x}, \bar{y})$ which does, meaning that in $\mathfrak{C}_{T}$ there are parameters $\left\{\bar{a}_{\eta}: \eta \in{ }^{\omega>} 2\right\rangle, \ell\left(\bar{a}_{\eta}\right)=\ell(y)$, such that:

(1) For each $\eta \in{ }^{\omega} 2$, the set $\left\{\varphi\left(\bar{x}, \bar{a}_{\eta \uparrow \ell}\right): \ell<\omega\right\}$ is consistent.

(2) For any two incomparable $\eta, \nu \in{ }^{\omega>} 2$, the set $\left\{\varphi\left(\bar{x}, \bar{a}_{\eta}\right), \varphi\left(\bar{x}, \bar{a}_{\nu}\right)\right\}$ is inconsistent.

Shelah in [18] had defined an order on theories, a natural weakening of Keisler's order: $T_{1} \triangleleft_{*} T_{2}$, which holds, roughly speaking, if there is a third theory $T_{*}$ which interprets both $T_{1}$ and $T_{2}$ and whose models $M_{*}$ have the property that if the reduct to $\tau\left(T_{2}\right)$ is saturated, so is the reduct to $\tau\left(T_{1}\right)$. [See Definition 6.7 below.] This was studied and developed more extensively by Džamonja-Shelah [1] and ShelahUsvyatsov 24]. In the Shelah-Usvyatsov paper, building on work of Džamonja and Shelah, it was shown that:

Fact 6.2. (Shelah and Usvyatsov [24] 3.15(2), under $\mathrm{GCH}^{12}$ ) If $\mathrm{T}$ is $\mathrm{NSOP}_{2}$ then $T$ is necessarily non-maximal in $\triangleleft^{*}$.

In Shelah and Usvyatsov [24 and Džamonja and Shelah [1] it was asked:

Question 6.3 (Question 1.8 of [24]). Does $\triangleleft^{*}$-maximality characterize either $S O P_{3}$ or $\mathrm{SOP}_{2}$, maybe both?

Question 6.4. ([1] Question 3.1) Does SOP 2 imply $\triangleleft^{*}$-maximality?

Here we settle the question, giving a positive answer: it characterizes $\mathrm{SOP}_{2}$. Now let us explain the connection to Keisler's order which motivates this work and our solution. $\mathrm{SOP}_{2}$ is a property which is not yet well understood and was not known, prior to the present paper, to be a dividing line. Recently, however, we proved the following theorem:

Theorem E. (Malliaris and Shelah [8] Theorem 11.11) Any theory with $S_{2} P_{2}$ is maximal in Keisler's order.

We conjecture there that $\mathrm{SOP}_{2}$ characterizes the maximum Keisler class. In the current section, we give strong evidence for this conjecture by proving the result for the order $\triangleleft^{*}$, which refines Keisler's order.

\footnotetext{
${ }^{12}$ This hypothesis is missing from the statement in [24, but that proof quotes [1] 3.2, which assumes relevant instances of $\mathrm{GCH}$.
} 
We now state the main result of this section: we prove that $T$ is $\triangleleft^{*}$-maximal if and only if it has $\mathrm{SOP}_{2}$ (Theorem 6.14 below). In light of Fact 6.2 , it suffices to prove that any theory with $\mathrm{SOP}_{2}$ is $\triangleleft^{*}$-maximal. Since $\triangleleft^{*}$ refines Keisler's order, even though it is not known whether it is a strict refinement, it is not sufficient to quote Theorem E; rather, we use the technology of cofinality spectrum problems developed for the proof of Theorem E

Given this result, it is natural to try to say more about the dividing line at $\mathrm{SOP}_{2}$, which we do in 88

Convention 6.5. Throughout this section $T$ denotes a complete countable firstorder theory.

We now review the "interpretability order" $\triangleleft^{*}$, introduced in Shelah 18 Definition 2.10 as a natural weakening of Keisler's order. We first need a definition of "interpretation."

Definition 6.6. (Interpretations, c.f. 1 1.1) Let $T_{0}$ and $T_{*}$ be complete first-order theories. Suppose that

$$
\bar{\varphi}=\left\langle\varphi_{R}\left(\bar{x}_{R}\right): R \text { a predicate or function symbol of } \tau\left(T_{0}\right) \text {, or }=\right\rangle
$$

is such that each $\varphi_{R}\left(\bar{x}_{r}\right) \in \tau\left(T_{*}\right)$.

(1) For any model $M_{*} \models T_{*}$, we define the model $N=M_{*}^{[\bar{\varphi}]}$ as follows:

- $N$ is a $\tau\left(T_{0}\right)$-structure

- $\operatorname{dom}(N)=\left\{a: M_{*} \models \varphi_{=}(a, a)\right\} \subseteq M_{*}$

- for each predicate symbol $R$ of $\tau\left(T_{0}\right), R^{N}=\left\{\bar{a}: M_{*} \models \varphi_{R}[\bar{a}]\right\}$

- for each function symbol $f$ of $\tau\left(T_{0}\right)$ and each $b \in N, N=" f(\bar{a})=b$ " iff $M_{*}=\varphi_{f}(\bar{a}, b)$, and $M_{*} \models$ " $\varphi_{f}(\bar{a}, b) \wedge \varphi_{f}(\bar{a}, c) \Longrightarrow b=c "$.

Note that by the last clause, we may restrict to vocabularies with only predicate symbols.

(2) Say that $\bar{\varphi}$ is an interpretation of $T_{0}$ in $T_{*}$ if:

- each $\varphi_{R}\left(\bar{x}_{r}\right) \in \tau\left(T_{*}\right)$

- for any model $M_{*}=T_{*}$, we have that $M_{*}{ }^{[\bar{\varphi}]}=T_{0}$

(3) If there exists $\bar{\varphi}$ which is an interpretation of $T_{0}$ in $T_{*}$, say simply that " $T_{*}$ interprets $T_{0} . "$

Definition 6.7. (The interpretability order $\triangleleft^{*}$, c.f [1] 1.2)

(1) Let $T_{0}, T_{1}$ be complete first-order theories and $\lambda$ an infinite regular cardinal. We say that $T_{0} \triangleleft_{\lambda}^{*} T_{1}$ if there exists a theory $T_{*}$ such that:

(a) $T_{*}$ interprets $T_{0}$, witnessed by $\overline{\varphi_{0}}$, and $T_{1}$, witnessed by $\overline{\varphi_{1}}$, where "interprets" is in the sense of 6.6

(b) For every model $M_{*}=T_{*}$, if $M_{*}^{\left[\overline{\varphi_{0}}\right]}$ is $\lambda$-saturated, then $M_{*}^{\left[\bar{\varphi}_{1}\right]}$ is $\lambda$-saturated

(2) We say that $T_{0} \triangleleft^{*} T_{1}$ if $T_{0} \triangleleft_{\lambda}^{*} T_{1}$ for all large enough regular $\lambda$.

Discussion 6.8. Definition 6.7 is stated for regular cardinals, but it also makes sense for singular cardinals. 
As ultrapowers commute with reducts and the choice of index models is irrelevant ${ }^{13}$ clearly $\triangleleft^{*}$-equivalence refines $\triangleleft$-equivalence [equivalence in Keisler's order]. A priori, one would expect that $\triangleleft^{*}$ is much weaker but this is not known to be the case. In fact, the known Keisler classes (i.e. of stable theories) coincide with those for $\triangleleft^{*}$ by 18 2.11.

We will use a result parallel to that familiar from Keisler's order:

Fact 6.9. (Shelah [18 2.11 p. 23) Any theory with the strict order property is $\triangleleft^{*}$-maximal $[$ i.e. -maximum].

Claim 6.10. Let $\mathbf{s}$ be a csp so $\mathfrak{p}_{\mathbf{s}}=\mathfrak{t}_{\mathbf{s}}$. Suppose $\mathbf{a} \in \operatorname{Or}(\mathbf{s})$ has a $\left(\mathfrak{t}_{\mathbf{s}}, \mathfrak{t}_{\mathbf{s}}\right)$-cut. Then $\mathcal{T}_{\mathbf{a}}$ witnesses treetops.

Proof. (Included for completeness, this argument simply adapts the proof of [8] Lemma 6.1, which proved that in this case $\mathcal{T}_{\mathbf{a} \times \mathbf{a}}$ witnesses treetops, to show that $\mathcal{T}_{\mathbf{a}}$ witnesses treetops.) Choose a sequence $\left(\left\langle a_{\alpha}: \alpha<\mathfrak{t}_{\mathbf{s}}\right\rangle,\left\langle b_{\alpha}: \alpha<\mathfrak{t}_{\mathbf{s}}\right\rangle\right)$ witnessing the cut. By induction on $\alpha<\mathfrak{t}_{\mathbf{s}}=\mathfrak{p}_{\mathbf{s}}$ let us choose a path through $\mathcal{T}_{\mathbf{a}}$ satisfying the following.

- for each $\alpha, c_{\alpha}$ belong: ${ }^{14}$ to the definable subtree of $\mathcal{T}_{\mathbf{a}}$ consisting of elements $x$ such that: for each $n<\max \operatorname{dom}(x), x(n)$ codes a pair [i.e. is of the form $(a+b)^{2}+a$ for elements $\left.a<_{\mathbf{a}} b \in X_{\mathbf{a}}\right]$, and if $m<n<\max \operatorname{dom}(x)$ and $x(m)=\left\langle a_{1}, b_{1}\right\rangle$ and $x(n)=\left\langle a_{2}, b_{2}\right\rangle$ then $a_{1}<_{\mathbf{a}} a_{2}<_{\mathbf{a}}<b_{2}<_{\mathbf{a}}<b_{1}$.

- $\beta<\alpha \Longrightarrow c_{\beta} \triangleleft c_{\alpha}$

- for each $\alpha, n_{\alpha}:=\max \operatorname{dom}\left(c_{\alpha}\right)$

- for each $\alpha, c_{\alpha}\left(n_{\alpha}\right)=\left\langle a_{\alpha}, b_{\alpha}\right\rangle$.

The construction of this tree follows the template of [8]. At successor steps, we concatenate. At limit steps $\alpha<\mathfrak{t}_{\mathbf{s}}$, we first choose an upper bound $c_{*}$ for the sequence built so far, by definition of $\mathfrak{t}_{\mathbf{s}}$. Let $n_{*}=\max \operatorname{dom}\left(c_{*}\right)$. Then since $X_{\mathbf{a}}$ is pseudofinite, the nonempty, bounded set $\left\{n<n_{*}: c_{*}(n)=\langle a, b\rangle\right.$ and $a<_{\mathbf{a}} a_{\alpha}$ and $\left.b_{\alpha}<_{\mathbf{a}} b\right\}$ has a maximal element $n_{* *}$. Then let $c_{\alpha}=c_{*} \uparrow_{n_{* *}} \frown\left\langle a_{\alpha}, b_{\alpha}\right\rangle$.

Having completed the construction of the sequence, notice that it is unbounded in $\mathcal{T}_{\mathbf{a}}$, because if it were to have an upper bound $c_{\star}$ then either of the elements in the pair coded by $c_{\star}\left(\max \operatorname{dom}\left(c_{*}\right)\right)$ would realize our original cut, contradiction. This completes the proof.

We will use one further fact about trees in CSPs, which explains that $\operatorname{Tr}(\mathbf{s})$ is quite robust.

Fact 6.11 ([8] Claim 2.13). If $\left(\mathcal{T}, \triangleleft_{\mathbf{a}}\right)$ is a definable subtree of $\left(\mathcal{T}_{\mathbf{a}}, \triangleleft_{\mathbf{a}}\right)$ and $\left\langle c_{\alpha}\right.$ : $\alpha<\kappa\rangle$ is a $\triangleleft_{\mathbf{a}}$-increasing sequence of elements of $\mathcal{T}$, then $\left\langle c_{\alpha}: \alpha<\kappa\right\rangle$ has an upper bound in $\mathcal{T}$ if and only if it has an upper bound in $\mathcal{T}_{\mathbf{a}}$.

\footnotetext{
${ }^{13}$ Keisler [4] proved that if $\mathcal{D}$ is a regular ultrafilter on $\lambda$ and $M \equiv N$ in a countable language then $M^{\lambda} / \mathcal{D}$ is $\lambda^{+}$-saturated iff $N^{\lambda} / \mathcal{D}$ is $\lambda^{+}$-saturated.

${ }^{14}$ Alternately, rather than asking that $c_{\alpha}$ code a pair, ask that if $t_{1}<t_{2}<t_{3}$ are successive elements of $\lg \left(c_{\alpha}\right)$ then either $c_{\alpha}\left(t_{1}\right)<c_{\alpha}\left(t_{3}\right)<c_{\alpha}\left(t_{2}\right)$ or else $c_{\alpha}\left(t_{2}\right)<c_{\alpha}\left(t_{3}\right)<c_{\alpha}\left(t_{1}\right)$. Then at limit steps, the condition is that the interval defined by $\left(c_{\alpha}(n), c_{\alpha}(n+1)\right)$ includes $a_{\alpha}$.
} 
Definition 6.12. Say that a tree $\mathcal{T}_{\mathbf{a}} \in \operatorname{Tr}(\mathbf{s})$ witnesses treetops if there is in $\mathcal{T}_{\mathbf{a}}$ a strictly increasing sequence of cofinality $\mathfrak{t}_{\mathbf{s}}$ with no upper bound, i.e. the infinite minimum is attained in $\mathcal{T}_{\mathbf{a}}$.

Fact 6.11 shows that if $\mathcal{T}^{\prime}$ is a definable subtree of $\mathcal{T}_{\mathbf{a}}, \mathcal{T}_{\mathbf{a}}$ witnesses treetops and there is an infinite increasing sequence in $\mathcal{T}^{\prime}$ with no upper bound, then $\mathcal{T}^{\prime}$ witnesses treetops. But there may not be such a sequence, as e.g. in the trivial case when $\mathcal{T}^{\prime}$ consists just of the root.

Lemma 6.13. Let $T_{1}$ be any theory with $S O P_{2}$. Then $T_{1}$ is maximal in $\triangleleft^{*}$.

Proof. Let $T_{0}$ be the theory $T h\left(\omega+\omega^{*}\right)$ of an infinite discrete linear order with a first and last element. As just explained, it will suffice to show that there is a theory $T_{*}$ with the following properties.

For any $M_{*}=T_{*}$,

- there exists an interpretation of $T_{1}$ in $M_{*}$, denoted $M_{*}{ }^{\left[\bar{\varphi}_{1}\right]}$

- there exists an interpretation of $T_{0}$ in $M_{*}$, denoted $M_{*}{ }^{\left[\bar{\varphi}_{0}\right]}$

- for any infinite regular cardinal $\lambda$, if $M_{*}{ }^{\left[\varphi_{1}\right]}$ is $\lambda$-saturated then $M_{*}{ }^{\left[\varphi_{0}\right]}$ is $\lambda$-saturated.

We build the theory $T_{*}$ in several steps. Let $\varphi(x, y)$ be a formula of $T_{1}$ which has $S O P_{2}$. Let $n_{\varphi}=\ell(y)$; here and elsewhere, we may omit overlines. Without loss of generality, $\tau\left(T_{1}\right)$ has only predicates. To begin, fix:

- a model $M_{1} \models T_{1}$ and a set of parameters $\left.\left\langle a_{\eta}: \eta \in \omega\right\rangle \omega\right\rangle$ witnessing $S O P_{2}$ for $\varphi$. [Recall that by compactness, we may assume our tree witnessing $\mathrm{SOP}_{2}$ is infinitely branching, with paths consistent and incomparable nodes inconsistent.]

- a model $M_{2} \models\left(\mathcal{H}\left(\aleph_{1}\right), \in\right)$. Without loss of generality, the domains of $M_{1}$ and $M_{2}$ are disjoint, and the relation $\epsilon$ does not appear in $\tau\left(M_{1}\right)$.

Let $M$ be the model which is the disjoint union of $M_{1}$ and $M_{2}$, expanded by the additional relations and functions $\{N, P, F\}$ as follows and no additional structure. (Without loss of generality, these symbols do not occur in $\tau\left(M_{1}\right)$ or $\tau\left(M_{2}\right)$.)

- $N^{M}$ names $\omega$ in $M_{2}$.

- $P^{M}$ names $\left\{a_{\eta}: \eta \in{ }^{n>} n\right\} \subseteq \operatorname{dom}\left(M_{1}\right)$.

- $F^{M}$ is a unary function with domain ${ }^{\omega>} \omega \subseteq M_{2}$ and range $P^{M} \subseteq M_{1}$, such that $F^{M}(\eta)=a_{\eta}$.

For each $n<\omega$, let $M_{n}$ be the model $M$ expanded further by the constant $c$ where $c^{M_{n}}=n \in \omega=N^{M}=N^{M_{n}}$. Let $\mathcal{D}$ be a nonprincipal ultrafilter on $\omega$. Let $N_{*}=\prod_{n} M_{n} / \mathcal{D}$ and let $T_{*}=T h\left(N_{*}\right)$. This completes the construction of $T_{*}$, the theory of the ultraproduct as just defined. Note that $\tau\left(T_{*}\right)=\tau\left(N_{*}\right)=\tau\left(M_{1}\right) \cup$ $\tau\left(M_{2}\right) \cup\{N, P, F, c\}$. In any model $M$ of $T_{*}$, the constant $c^{M}$ is a nonstandard element of $N^{M}$, i.e. of the (nonstandard) copy of $\omega$.

In addition to $T_{*}$ and $N_{*}$ as just defined, let $M_{*}$ be an arbitrary but fixed model of $T_{*}$ which satisfies: $M_{*} \Gamma_{\tau\left(T_{1}\right)}$ is $\lambda$-saturated. Note that $T_{*}$ interprets $T_{0}$ and $T_{1}$, that is, there exist $\bar{\varphi}_{0}, \bar{\varphi}_{1}$ witnessing 6.6 This is because $\left.M_{*}\right|_{\tau\left(T_{1}\right)} \mid=T_{1}$ by construction, while $N_{0}:=N^{M_{*}}\left\lceil c^{M_{*}}=\left(\left\{a \in M_{*}: M_{*} \models a \in c\right\}, \in^{M_{*}}\right) \models T_{0}\right.$. 
To analyze saturation, we define a cofinality spectrum problem as follows. First, let $\operatorname{Or}^{0}(\mathbf{s})$ be the smallest set containing the set of all finite nonempty initial segments of $N^{M_{*}}$, in each case letting $d_{\mathbf{a}}=\max X_{\mathbf{a}}$. [We may use any non-empty order in $\mathcal{H}\left(\aleph_{0}\right)$.] For each $\mathbf{a}, \mathbf{b} \in \operatorname{Or}^{0}(\mathbf{s})$, let $\mathbf{a} \times \mathbf{b}$ be the definable, pseudofinite linear order on $X_{\mathbf{a}} \times X_{\mathbf{b}}$ given by the Gödel pairing function, and let $d_{\mathbf{a} \times \mathbf{b}}=\left(d_{\mathbf{a}}, d_{\mathbf{b}}\right)$. Let $\operatorname{Or}(\mathbf{s})$ be the closure as just described of $\operatorname{Or}^{0}(\mathbf{s})$ under Cartesian product, as well as initial segment. [If $\mathbf{b}$ is an initial segment of $\mathbf{a}$, define $d_{\mathbf{b}}=\min \left\{\max X_{\mathbf{b}}, d_{\mathbf{a}}\right\}$.]

Suppose for a moment that $M_{*}=N_{*}$, i.e. suppose we are really in the case of the ultrapower. Then for each $\mathbf{a} \in \operatorname{Or}(\mathbf{s}), \varphi_{\mathbf{a}}$ is a formula $\varphi(x, y, z)$ over the empty set. Because we are in $N_{*}$, for each suitable parameter $\bar{a}$, the set $\varphi(x, x, \bar{a})$ is contained in the ultraproduct of finite sets. Let $\psi(w, z)$ be such that for each suitable $\bar{a} \in{ }^{\ell(\bar{z})} M_{n}$ [though by construction, we can restrict to the case where $\left.\bar{a} \subseteq \mathbb{N}^{M_{n}}\right]$ we have that $\mathcal{T}_{\varphi, \bar{a}}=\{\eta: M \mid=\psi(\eta, \bar{a})\}$ is the set of finite sequences of members of the finite set $X_{\varphi, \bar{a}}=\{b: M \models \varphi(b, b, \bar{a})\}$ of length $<\max X_{\varphi, \bar{a}}$. Let $\triangleleft=\left\{(\eta, \nu): \eta, \nu \in \mathcal{T}_{\varphi, \bar{a}}\right.$ and $\eta$ is an initial segment of $\left.\nu\right\}$. These are definable in $M_{n}$ by the choice of its theory. The length and evaluation functions $\mathrm{lg}$ and val can be defined likewise. By Łos' theorem, these formulas will define the appropriate trees in the ultrapower. Thus, we get a cofinality spectrum problem. Moreover, for each given $\varphi_{\mathbf{a}}$, each $\bar{a}$ of length $\ell(z)$, and each $t<\omega$, we have that in each index model, the tree $\mathcal{T}=\mathcal{T}_{\varphi, \bar{a}[t]}$ is finite, so its flattening $\left(\mathcal{S},<_{\mathcal{S}}\right)$ [in the sense of 2.2 above] can be injectively mapped into $N^{M_{n}}$. That is, $M_{n}=$ "there exists an injective order-preserving map of $\left(\mathcal{S},<_{\mathcal{S}}\right)$ into $N$ ". As the flattening is uniformly definable from $\psi$, and we are in a model with sufficient set theory, such an injection exists also in the ultrapower and so $\mathbf{s}$ has (strong) exponentiation.

Returning to the case of arbitrary $M_{*} \equiv N_{*}$, as we are in a model with sufficient set theory, note that the existence of such associated trees and injections are elementary properties of each given $\varphi$ and $\psi$. Thus, also in this more general case, $\mathbf{s}$ is a cofinality spectrum problem with exponentiation.

Having defined $\mathbf{s}$, we now prove that $\mathfrak{t}_{\mathbf{s}} \geq \lambda$. First we verify that it is sufficient to look at one-dimensional trees, i.e. that treetops are witnessed by some $\mathcal{T}_{\mathbf{a}}$ where $X_{\mathbf{a}} \subseteq N^{M_{*}}$. By Theorem 2.10, there is a $\left(\mathfrak{t}_{\mathbf{s}}, \mathfrak{t}_{\mathbf{s}}\right)$-cut in some $X_{\mathbf{a}}, \mathbf{a} \in \operatorname{Or}(\mathbf{s})$. If $X_{\mathbf{a}}$ is one-dimensional, then by Claim 6.10, we finish. If not, $X_{\mathbf{a}}$ is contained in some finite Cartesian power of $N^{M_{*}}$. Let $f: X_{\mathbf{a}} \rightarrow N^{M_{*}}$ be a definable injection, given by applying the Gödel pairing function finitely many times. By the definition of s, this is an order preserving map. (This appeal to the definition is not necessary: we could simply use that such a map is an injection of sets, so by Conclusion 7.4 of the next section, there is an injective order-preserving map into some one-dimensional $X_{\mathbf{b}}$.) Necessarily $X_{\mathbf{b}}$ will have a $\left(\mathfrak{t}_{\mathbf{s}}, \mathfrak{t}_{\mathbf{s}}\right)$-cut, so again by Claim 6.10, we finish.

So to show $\mathfrak{t}_{\mathbf{s}} \geq \lambda$, it will suffice to show that for any one-dimensional $\mathbf{a}$, and any $\kappa<\lambda$, any $\kappa$-indexed strictly increasing sequence $\left\langle\eta_{\alpha}: \alpha<\kappa\right\rangle$ in $\mathcal{T}_{\mathbf{a}}$ has an upper bound. Recall the constant $c$ from the signature of $N_{*}$ and $M_{*}$. Since $M_{*} \equiv N_{*}$, any one-dimensional $X_{\mathbf{a}}$ is contained in a nonstandard initial segment, i.e. for some $m, \mathbb{N}^{M_{*}}=" c \leq m$ and $X_{\mathbf{a}} \subseteq M_{*} \uparrow m$ ". By Fact 6.11, without loss of generality $X_{\mathbf{a}}$ is the full initial segment below $m$ and $\mathcal{T}_{\mathbf{a}}$ is the full tree ${ }^{m>} m$. In $\left(\mathcal{H}\left(\aleph_{0}\right), \epsilon\right)^{M_{*}}$ we 
define $\mathcal{T}_{\mathbf{a}}=\left({ }^{m>} m, \triangleleft\right)$ and let

$$
\left.\psi(y, m):=\left(\exists \eta \in \mathcal{T}_{\mathbf{a}}\right)(F(\eta)=y)\right)
$$

define the subset of $P^{M_{*}}$ corresponding to the image $F\left(\mathcal{T}_{\mathbf{a}}\right)$. Now if $\bar{\eta}=\left\langle\eta_{\alpha}: \alpha<\kappa\right\rangle$ is increasing in $\mathcal{T}_{\mathbf{a}}$, then recalling the $S O P_{2}$-formula $\varphi$ from the beginning of the proof,

$$
p_{\bar{\eta}}=\left\{\varphi\left(x, F\left(\eta_{\alpha}\right)\right): \alpha<\kappa\right\}
$$

is a consistent partial typ ${ }^{15}$ in $M_{*} \uparrow \tau\left(T_{1}\right)$. By the assumption of $\lambda$-saturation in that signature, some $d \in M_{*}$ realizes $p$, hence

$$
\left\{\eta \in \mathcal{T}_{\mathbf{a}}: \varphi[d, F(\eta)]\right\}
$$

is a subset of a branch of $\mathcal{T}_{\mathbf{a}}$ by definition of $\mathrm{SOP}_{2}$ and of $F$, and is definable in $M_{*}$. Then the set

$$
\left\{a \in X_{\mathbf{a}}: a<d_{\mathbf{a}} \wedge(\exists \eta)\left(\eta \in \mathcal{T}_{\mathbf{a}} \wedge \varphi[d, F(\eta)] \wedge a \in \operatorname{dom}(\eta)\right)\right\}
$$

of lengths of such elements is a definable, nonempty, bounded subset of $X_{\mathbf{a}}$, so contains a greatest element $a_{*}$. Any $\eta$ along the distinguished branch whose domain contains $a_{*}$ will be an upper bound for $\bar{\eta}$. This completes the proof that $\mathfrak{t}_{\mathrm{s}} \geq \lambda$.

Recall that we had set $N_{0}:=N^{M_{*}} \uparrow c^{M_{*}}$ as the domain for our interpretation of $T_{0}$ in $M_{*}$. Since $\mathbf{s}$ is a CSP with exponentiation, $\mathfrak{p}_{\mathbf{s}}=\mathfrak{t}_{\mathbf{s}}=\lambda$ by Theorem 2.10. By definition of $\mathfrak{p}_{\mathbf{s}}$, there are no $\left(\kappa_{1}, \kappa_{2}\right)$-cuts in any $X_{\mathbf{a}}$, for $\mathbf{a} \in \operatorname{Or}(\mathbf{s})$ and $\kappa_{1}, \kappa_{2}<\mathfrak{t}_{\mathbf{s}}$. This in particular is true for a with $X_{\mathbf{a}}=\left\{a: M_{*} \models a<c\right\}$ and $d_{\mathbf{a}}=\max X_{\mathbf{a}}$. In fact, for arbitrarily large $a \in N^{M_{*}}, \operatorname{Or}(\mathbf{s})$ contains a such that $X_{\mathbf{a}} \supseteq N \uparrow_{\leq a}$, in which, therefore, we have no $\left(\kappa_{1}, \kappa_{2}\right)$-cuts.

Finally, let us prove that $M_{*}{ }^{\left[\varphi_{0}\right]}$, i.e. the $\tau_{0}$-submodel whose domain is $N_{0}$, is $\lambda$ saturated. We know that for models of $T_{0}$, every formula is a Boolean combination of the formulas $x=y, x<y$, and $\varphi_{k}(x, z)=\left(\exists^{! k} y\right)(x<y<z)$. Thus $N_{0} \models T_{0}$ is $\lambda$-saturated iff every cut $\left(C_{1}, C_{2}\right)$ of $N_{0} \subseteq N^{M_{*}}$ of cofinality $\left(\kappa_{1}, \kappa_{2}\right)$ is filled, where $\kappa_{1}, \kappa_{2}$ are regular and $\kappa_{1}+\kappa_{2} \leq \lambda$. This was proved in the previous paragraph.

This completes the proof.

Theorem 6.14 (GCH). $T$ is $\triangleleft^{*}$-maximal if and only if it has $\mathrm{SOP}_{2}$.

Proof. By Fact 6.2 (which assumes relevant instances of GCH) and Lemma6.13.

\section{UsEFul TOOLS AND ADDITIONAL DEFINITIONS}

Before turning to a structure theory for $\mathrm{NSOP}_{2}$, we prove several additional facts about CSPs: Claim 7.3 and Conclusion 7.4, which show that from a suitable bijection of sets, we can recover an order-isomorphism. It follows that the assumption that the order on all pairs was given by the Gödel pairing function in the CSP constructed in Lemma 6.13 could be weakened, as mentioned in that proof. Tying

\footnotetext{
${ }^{15}$ The fact that comparability of elements in the domain of $F$ is reflected in the consistency of their images will carry over from the models $M_{n}$ to $N_{*}$ by Łos' theorem and from there to $M_{*}$ by elementary equivalence.
} 
up loose ends, we show that one of the main consequences of 1.1 (6) can be recovered in weak hereditary CSPs, Claim 7.5, and discuss the barrier to fully recovering 8 for such CSPs in 7.6. Finally, we include a definition of "strong" CSPs, natural when the underlying model $M_{1}^{+}$is totally ordered.

Definition 7.1. Let $\mathbf{b} \in \operatorname{Or}(\mathbf{s})$. We say that $Z \subseteq X_{\mathbf{b}}$ is small in $X_{\mathbf{b}}$ if:

(a) there is some definable $V, Z \subseteq V \subset X_{\mathbf{b}}$ such that $M_{1}^{+} \models$ "there does not exist $x \in \mathcal{T}_{\mathbf{b}}$ such that $x$ is a bijection from $X_{\mathbf{b}}$ into $V$."

(b) if $Z \neq \emptyset$, then $\max (Z)<d_{\mathbf{b}}$

When $Z \subseteq X_{\mathbf{b}}$ is small in $X_{\mathbf{b}}$ and is an initial segment, we call it a small initial segment.

Remark 7.2. Condition 7.1(b) allows for concatenation.

Claim 7.3. Suppose we are given a weak cofinality spectrum problem $\mathbf{s}, \mathbf{b} \in \operatorname{Or}(\mathbf{s})$, $h \in M_{1}^{+},\left(W,<_{W}\right)$ a definable and pseudofinite linear order, and $Z$ a small initial segment of $Y_{\mathbf{b}}$, such that:

$M_{1}^{+}=$" $h$ is a partial bijection with $W \subseteq \operatorname{dom}(h)$, range $(h) \subseteq Z$ "

Then $\left(W,<_{W}\right)$ is internally order-isomorphic to an initial segment of $Y_{\mathbf{b}}$.

Proof. Let $\left(W^{*},<_{W^{*}}\right)$ denote the definable set $h(W) \subseteq X_{\mathbf{b}}$ with the definable linear order given by $x<_{W^{*}} y \Longleftrightarrow h^{-1}(x)<_{W} h^{-1}(y)$. This allows us to identify $\left(W^{*},<_{W^{*}}\right)$ definably with $\left(W,<_{W}\right)$, and now we prove that $\left(W^{*},<_{W^{*}}\right)$ is internally order-isomorphic to an initial segment of $\left(X_{\mathbf{b}}, \leq_{\mathbf{b}}\right)$.

Let $\mathcal{T}$ be the definable subtree of $\mathcal{T}_{\mathbf{b}}$ given by $\varphi(x)$, which says: " $x \in \mathcal{T}_{\mathbf{b}}$ is a one to one order preserving function from an initial segment of $\left(X_{\mathbf{b}}, \leq_{\mathbf{b}}\right)$ onto an initial segment of $\left(W^{*},<_{W^{*}}\right)$ ". Thus if $a<_{\mathbf{b}} b$ are in $\operatorname{dom}(x)$ then $x(a)<_{W^{*}} x(b)$.

First, note that the set $\mathcal{F}=\{x \in \mathcal{T}: \varphi(x)\}$ is linearly ordered. This is because any two elements $x, x^{\prime} \in \mathcal{T}$ must agree on the first element of their domain; and if neither of $x, x^{\prime}$ extends the other, then the set in their common domain on which they agree is nonempty and definable. But if the last element of this set is not $\max \operatorname{dom}(x)$, we get a contradiction since the order is pseudofinite.

Consider the subset of $X_{\mathbf{b}}$ given by:

$$
\left\{a \in X_{\mathbf{b}} \text { : there is } x \text { such that } \varphi(x) \text { and } a \in \operatorname{dom}(x)\right\}
$$

As this subset is nonempty and definable, by assumption it has a last element $a_{*} \in X_{\mathbf{b}}$. Let $x_{*} \in \mathcal{T}$ be a function witnessing this, i.e. such that $a_{*} \in \operatorname{dom}\left(x_{*}\right)$. Necessarily $x_{*}=\max \mathcal{F}$.

There are three cases.

Case 1. The desired case: $\operatorname{range}\left(x_{*}\right)=W^{*}$.

Case 2. Not case 1 , but $\operatorname{dom}\left(x_{*}\right)=X_{\mathbf{b}}$. Then

$$
M_{1}^{+}=" x_{*} \in \mathcal{T}_{\mathbf{b}} \text { is an injection of } X_{\mathbf{b}} \text { into } Z \text { " }
$$

contradicting Definition 7.1 . 
Case 3. Not case 1 or 2 , so $\operatorname{dom}\left(x_{*}\right) \subsetneq X_{\mathbf{b}}$ and $\operatorname{range}\left(x_{*}\right) \subsetneq W^{*}$. By Definition 7.1 and the hypotheses of the Claim, "not case 2" implies $\lg \left(x_{*}\right)<d_{\mathbf{b}}$. Writing $S$ for successor, note that as the orders are pseudofinite, the function $\left.x_{*}\right\urcorner\left\langle S\left(f\left(a_{*}\right)\right)\right\rangle$ is well defined (meaning the function extending $x_{*}$ by the additional condition $S\left(a_{*}\right) \mapsto S\left(f\left(a_{*}\right)\right)$ is well defined). We may concatenate, so this new function belongs to $\mathcal{T}$, contradicting the choice of $a_{*}$.

As Cases 2 and 3 are contradictory, we are necessarily in Case 1, which completes the proof.

Conclusion 7.4. Suppose that $\mathbf{s}$ is a weak cofinality spectrum problem, and:

(1) $N \subseteq M_{1, \mathbf{s}}$ is definable and linearly ordered.

(2) Arbitrarily large initial segments of $N$ are orders for $\mathbf{s}$, more precisely, there is $\psi \in \Delta$ so that for any $a \in N$ there is $\mathbf{a} \in \operatorname{Or}(\mathbf{s})$ with:

$\{b \in N: \models b \leq a\} \subseteq\left\{b \in M_{1, s}: \models \psi(b, b, a)\right\}=X_{\mathbf{a}} \subseteq N$ and $a<d_{\mathbf{a}}$.

(3) Cardinality of initial segments of $N$ grows internally, more precisely, letting $\theta=\theta(w, y)$ define the tree associated to $\psi\left(x_{1}, x_{2}, y\right)$, we have that $M_{1, \mathbf{s}}=$ " $\left(\forall y_{0} \in N\right)\left(\exists y_{1} \in N\right)$ (there does not exist $z \in \theta\left(w, y_{1}\right)$ such that $z$ is a bijection from $\psi\left(x, x, y_{1}\right)$ into $\left.\psi\left(x, x, y_{0}\right)\right)$ ".

Let $\left(W,<_{W}\right)$ be a definable, pseudofinite linear order in the model. If there is an internal injection $f$ of sets from $W$ into some initial segment of $N$, then for some $\mathbf{b} \in \operatorname{Or}(\mathbf{s})$ with $X_{\mathbf{b}} \subseteq N$ there exists an internal order-isomorphism $g$ from $\left(W,<_{W}\right)$ onto an initial segment of $Y_{\mathbf{b}}$.

Proof. By the hypothesis, we can find $\mathbf{b}_{0}$ such that $X_{\mathbf{b}_{0}} \supseteq f(W)$, and $d_{\mathbf{b}_{0}}>$ $\max (f(W))$. Let $\psi(z)$ be the formula in $\Delta$ defining the pseudofinite linear order corresponding to the initial segment below $z$, and let $\theta(z)$ define its associated tree. Then $M_{1, \mathbf{s}} \models$ " $(\exists z)$ (there does not exist $x \in \theta(z)$ such that $x$ is a bijection from $\psi(z)$ into $\left.X_{\mathbf{b}_{0}}\right)$ ". Let $c$ be any such $z$. Again by hypothesis, there is $\mathbf{b} \in \operatorname{Or}(\mathbf{s})$ such that $X_{\mathbf{b}} \supseteq\{x: x \leq c\}$ and $d_{\mathbf{b}}>c$. Now apply Claim 7.3 .

Claim 7.5. If $\mathbf{s}$ is a nontrivial weak c.s.p. and is hereditarily closed, then there exist nontrivial $\mathbf{a}, \mathbf{a}^{\prime} \in \operatorname{Or}(\mathbf{s})$ which together satisfy $(* *)$ of 1.5 .

Proof. Let $\mathbf{b}$ (so also $\mathcal{T}_{\mathbf{b}}$ ) be nontrivial. In section 5 of [8], we showed that for any $\mathbf{b} \in \operatorname{Or}(\mathbf{s})$, it is possible to define addition, multiplication, and exponentiation on any element of $\operatorname{Or}(\mathbf{s})$, (that is, to define relations on $X_{\mathbf{b}}$ which have all the same properties as the graphs of these functions, except that they are possibly not total). This does not require any assumptions on pairing. This is done in the proof of 8 . Lemma 5.3, essentially as follows. Addition is given by: $\varphi_{+}(x, y, z)=\left(\exists \eta \in \mathcal{T}_{\mathbf{b}}\right)(\lg (\eta)=y \wedge \eta(0)=x \wedge \eta(y-1)=z \wedge(\forall i)(i<\lg (\eta) \Longrightarrow$ $\eta(S(i))=S(\eta(i)))$. [We omit the parameter $\bar{c}_{\mathbf{b}}$ for readability.] To obtain multiplication, $\varphi_{\times}(x, y, z)$, substitute " $(i<\lg (\eta) \Longrightarrow \eta(S(i))=\eta(i)+x)$ " as necessary, and define exponentation $\varphi_{\exp }(x, y, z)$ by substituting in the appropriate place " $(i<\lg (\eta) \Longrightarrow \eta(S(i))=\eta(i) \times x)$," i.e. requiring that the sequence increment by a factor of $x$. These are graphs of partial functions, which need not be total. 
We can therefore define " $x$ is a prime." Let $\varphi_{4}(x, y)$ assert that $x$ is the $y$ th prime by saying: $y>0 \wedge\left(\exists \eta \in \mathcal{T}_{\mathbf{b}}\right)(\lg (\eta)=y \wedge \eta(0)=2 \wedge \eta(y-1)=x \wedge(\forall i)(i<\lg (\eta) \Longrightarrow$ " $\eta(S(i))$ is the $\leq_{\mathbf{b}}$-least prime number strictly greater than $\left.\left.\eta(i) "\right)\right)$. Let $\varphi_{5}(x, n, m)$ assert that $x$ is divisible by the $n$th prime precisely $m$ times, by asserting the existence of $\eta \in \mathcal{T}_{\mathbf{b}}$ of length $m$ whose first element is $x$, whose subsequent elements decrease by a factor of the $n$th prime and whose last element has no more such factors. Let the formula $\varphi_{6}(x, \eta)$ assert that $x \in X_{\mathbf{b}}$ is a Gödel code for $\eta$ by stating: " $\eta \neq \emptyset, \eta \in \mathcal{T}_{\mathbf{b}}, x>2$ and for all $i<\lg (\eta)$, writing $m=\eta(i)$, we have that $x$ is divisible by the $i$ th prime precisely $m+1$ times". Let $\theta(x)$ assert that $(\forall y<x)(\exists z) \varphi_{+}(x, y, z) \wedge(\forall y<x)(\exists z) \varphi_{\times}(x, y, z) \wedge(\forall y<x)(\exists z) \varphi_{\exp }(x, y, z)$. Let $\psi(y)$ be the formula:

$(\forall x<y) \theta(x) \wedge\left(\forall \eta \in \mathcal{T}_{\mathbf{b}}\right)\left((\lg (\eta)<y \wedge(\forall i<\lg (\eta))(\eta(i)<y)) \Longrightarrow(\exists x)\left(\varphi_{6}(x, \eta)\right)\right)$

which asserts that Gödel codes exist for all functions from $X_{\mathbf{b}} \uparrow_{y}$ to itself.

For our present case, apply this as follows. Recall that $X_{\mathbf{b}}$ is pseudofinite.

First step: Find nonstandard $n_{*} \leq d_{\mathbf{b}}$ so that the definable set $Z$ of codes for pairs of elements of $\left[0, n_{*}\right]_{\mathbf{b}}$ is contained in $Y_{\mathbf{b}}$. Second step: Find $n_{* *} \in X_{\mathbf{b}}$, $n_{* *} \leq n_{*}$ still nonstandard, so that $X_{\mathbf{b}}$ contains all codes for functions from $\left[0, n_{* *}\right]_{\mathbf{b}}$ to itself. Third step: Let $m_{*}$ be maximal $\leq_{\mathbf{b}} n_{* *}$ such that $\left\langle m_{*}, m_{*}\right\rangle \leq n_{* *}$, so necessarily $m_{*}$ is nonstandard too. Let $\mathbf{a}_{1}$ be such that $X_{\mathbf{a}_{1}}=\left[0, n_{* *}\right]_{\mathbf{b}}$ and $d_{\mathbf{a}_{1}}=m_{*}$.

Now define a so that $X_{\mathbf{a}}=\left[0, m_{*}\right]_{\mathbf{b}}$ and $d_{\mathbf{a}}=m_{*}$. What about the desired tree $\mathcal{T}$ of functions from $X_{\mathbf{a}}$ to $X_{\mathbf{a}} \times X_{\mathbf{a}}$ ? Recalling that $d_{\mathbf{a}_{1}}=m_{*}$, this tree is naturally isomorphic to the definable sub-tree $\mathcal{T} \subseteq \mathcal{T}_{\mathbf{a}_{1}}$ whose elements are functions whose range consists only of codes for pairs of elements each of which are $\leq m_{*}$. By construction, the codes for elements of $\mathcal{T}_{\mathbf{a}_{1}}$ and therefore for elements of $\mathcal{T}$ form a definable subset of $X_{\mathbf{b}}$. Finally, let $\mathbf{a}^{\prime}=\mathbf{b}$.

This completes the proof.

Discussion 7.6. By a similar argument, in any weak CSP which is hereditarily closed, for some nontrivial $\mathbf{a} \in \operatorname{Or}(\mathbf{s})$ we have available nontrivial elements of $\operatorname{Or}(\mathbf{s})$ which can be thought of as canonically representing any given one of the finite Cartesian powers $\mathbf{a} \times \cdots \times \mathbf{a}$ with the desired ordering, e.g. one derived from repeated applications of the Gödel pairing function. However, as noted in Discussion 1.5, we don't a priori have Cartesian products of distinct $\mathbf{a}, \mathbf{b} \in \operatorname{Or}(\mathbf{s})$. This prevents us from obtaining the uniformity of functions such as lcf across all $X_{\mathbf{a}}$ which was necessary for the main theorems of 8 , to go through.

Definition 7.7 (Strong CSPs). Call $\mathrm{s}$ a strong CSP, or lexicographic CSP if the demands on Cartesian products from 1.1.(5) are replaced by ${ }^{16}$

(1) if $\mathbf{a}, \mathbf{b} \in \operatorname{Or}(\mathbf{s})$ then internally either $\left|Y_{\mathbf{a}}\right| \leq\left|Y_{\mathbf{b}}\right|$ or $\left|Y_{\mathbf{b}}\right| \leq\left|Y_{\mathbf{a}}\right|$.

(2) if $\mathbf{a} \in \operatorname{Or}(\mathbf{s})$ and $d \leq \mathbf{a} d_{\mathbf{a}}$ then there is $\mathbf{b} \in \operatorname{Or}(\mathbf{s})$ such that:

(a) $X_{\mathbf{b}}=X_{\mathbf{a}},<_{\mathbf{b}}=<_{\mathbf{a}}, d_{\mathbf{b}}=d$

(b) $\mathcal{T}_{\mathbf{b}}=\mathcal{T}_{\mathbf{a}}\left\lceil\left\{\eta:\left(\lg _{\mathbf{a}}(\eta) \leq d\right) \wedge(\forall n<\max \operatorname{dom}(\eta))(\eta(n) \leq d)\right\}\right.$

\footnotetext{
${ }^{16}$ So here $\Delta$ is retained.
} 
(3) if $\mathbf{a}, \mathbf{b} \in \operatorname{Or}(\mathbf{s})$ and $h$ is a definabl $1^{17}$ isomorphism from $\left(Y_{\mathbf{a}},<_{\mathbf{a}}\right)$ onto $\left(Y_{\mathbf{b}},<_{\mathbf{b}}\right)$ then there is $\mathbf{c}=\mathbf{a} \times \mathbf{b}$ such that $X_{\mathbf{c}}=X_{\mathbf{a}} \times X_{\mathbf{b}},<_{\mathbf{c}}$ is the lexicographic order, $d_{\mathbf{c}}=\left(d_{\mathbf{a}}, 0_{\mathbf{b}}\right)$, and $\mathcal{T}_{\mathbf{c}}$ is naturally defined.

Observation 7.8. Any strong CSP is a weak CSP, and the hereditary closure of a strong CSP is a strong CSP.

Claim 7.9. If $\mathbf{s}$ is a strong CSP which is hereditarily closed, then:

(a) $\mathbf{s}$ satisfies Property (**) of Discussion 1.5 .

(b) Moreover, for any nontrivial $\mathbf{a}, \mathbf{b} \in \operatorname{Or}(\mathbf{s})$, there is a nontrivial $\mathbf{c} \in \operatorname{Or}(\mathbf{s})$ with either $X_{\mathbf{c}}=X_{\mathbf{a}} \times Y_{\mathbf{b}}$ or $X_{\mathbf{c}}=X_{\mathbf{b}} \times Y_{\mathbf{a}}$.

(c) Suppose that for every $\mathbf{a} \in \operatorname{Or}(\mathbf{s})$ there is $\mathbf{a}^{\prime} \in \operatorname{Or}(\mathbf{s})$ such that $X_{\mathbf{a}}$ is internally isomorphic to a subset of $X_{\mathbf{a}^{\prime}}$ and $d_{\mathbf{a}^{\prime}}=\max \left(X_{\mathbf{a}^{\prime}}\right)$. Then $\mathbf{s}$ is closed under Cartesian products.

Proof. (1) By Claim 7.5 and Observation 7.8

(2) Fix nontrivial $\mathbf{a}, \mathbf{b} \in \operatorname{Or}(\mathbf{s})$. By condition 7.7 (1), without loss of generality, $\left|Y_{\mathbf{a}}\right| \leq\left|Y_{\mathbf{b}}\right|$ witnessed by an internal partial isomorphism $h$. Since Condition 7.7)(3) requires $h$ to be surjective, let $d=h\left(d_{\mathbf{a}}\right) \in Y_{\mathbf{b}}$. Since $\mathbf{s}$ is hereditarily closed and $d \leq d_{\mathbf{b}}$, apply Observation 1.12 to find $\mathbf{b}^{\prime \prime} \in \operatorname{Or}(\mathbf{s})$ such that $X_{\mathbf{b}^{\prime \prime}}=Y_{\mathbf{b}}$ and $d_{\mathbf{b}^{\prime \prime}}=d$. Now $h: Y_{\mathbf{a}} \rightarrow Y_{\mathbf{b}^{\prime \prime}}$ is onto, so by condition 1.7)(c) there is $\mathbf{c}=\mathbf{a} \times \mathbf{b}^{\prime \prime}$ with $X_{\mathbf{c}}=X_{\mathbf{a}} \times X_{\mathbf{b}^{\prime \prime}}=X_{\mathbf{a}} \times Y_{\mathbf{b}}$ and $d_{\mathbf{c}}$ is nonstandard, thus $\mathbf{c}$ is nontrivial.

(3) As $d_{\mathbf{a}^{\prime}}=\max \left(X_{\mathbf{a}^{\prime}}\right), d_{\mathbf{b}^{\prime}}=\max \left(X_{\mathbf{b}^{\prime}}\right)$, we have that $Y_{\mathbf{a}^{\prime}}=X_{\mathbf{a}^{\prime}}, Y_{\mathbf{b}^{\prime}}=X_{\mathbf{b}^{\prime}}$, and the previous condition (2) shows that their Cartesian product exists. The hypothesis of (3) allows us to find Cartesian products for any two elements of $\operatorname{Or}(\mathbf{s})$ by first isomorphically embedding them in suitable larger elements $\mathbf{a}^{\prime}, \mathbf{b}^{\prime}$, finding $\mathbf{c}^{\prime}=\mathbf{a}^{\prime} \times \mathbf{b}^{\prime}$ and then applying hereditary closure.

\section{TOWARdS A STRUCTURE THEORY FOR $\mathrm{NSOP}_{2}$}

In Section 6, we gave the first real evidence that the strong tree property $\mathrm{SOP}_{2}$ is a dividing line. Motivated by this result, we now look for the beginnings of a structure theory for $\mathrm{NSOP}_{2}$. The key objects are so-called higher formulas, defined using ultrafilters. The main results are first, Theorem 8.21, which characterizes $\mathrm{NSOP}_{2}$ in terms of few higher formulas; second, the Symmetry Lemma 8.15, which characterizes $\mathrm{NSOP}_{3}$ in terms of symmetric inconsistency for higher formulas; and third, Theorem 8.30 , which proves that $S O P_{2}$ is sufficient for a certain kind of exact saturation to fail.

Convention 8.1. Throughout this section, $T$ is a complete first order theory and $\mathfrak{C}=\mathfrak{C}_{T}$ is a monster model for $T$.

$S O P_{2}$ was defined in 6.1 above. $S O P_{3}$ was first defined in Shelah 18 as a weakening of the strict order property; note that in Definition 8.2 , the case where $\psi=\neg \varphi$ is the strict order property.

\footnotetext{
${ }^{17}$ Not necessarily via $\Delta$.
} 
Definition 8.2 ( [18 2.20, 24 1.3). Thas $\mathrm{SOP}_{3}$ if there is an indiscernible sequence $\left\langle\bar{a}_{i}: i<\omega\right\rangle$ and formulas $\varphi(\bar{x}, \bar{y}), \psi(\bar{x}, \bar{y})$ such that:

(1) $\{\varphi(\bar{x}, \bar{y}), \psi(\bar{x}, \bar{y})\}$ is contradictory.

(2) for each $k<\omega$, the following is a consistent partial type:

$$
\left\{\psi\left(\bar{x}, \bar{a}_{j}\right): j \leq k\right\} \cup\left\{\varphi\left(\bar{x}, \bar{a}_{i}\right): i>k\right\}
$$

(3) for $j<i$, the set $\left\{\varphi\left(\bar{x}, \bar{a}_{i}\right), \psi\left(\bar{x}, \bar{a}_{j}\right)\right\}$ is contradictory.

It is known that $S O P_{3}$ implies $S O P_{2}$ but it is open whether, on the level of theories, the converse is true; so it is possible that the Symmetry Lemma below will also characterize $S O P_{2}$. (Still, for pairs $(T, \Delta)$ the converse fails since $S O P_{2}$, $\mathrm{SOP}_{3}$ are known to be distinct at the level of formulas.)

We first look for a useful way to capture the asymmetry of $S O P_{3}$. This approach relates to the idea of "semi-definability" from [16] VII.4.

Definition 8.3. If $\mathcal{D}$ is an ultrafilter on $A \subseteq \mathfrak{C}^{m}$, then for any set $B \subseteq \mathfrak{C}$ we define:

$$
\operatorname{Av}(\mathcal{D}, B)=\{\psi(\bar{x} ; \bar{b}): \bar{b} \in B, \psi \in[\mathcal{L}],\{\bar{a} \in A: \models \psi(\bar{a}, \bar{b})\} \in \mathcal{D}\}
$$

so this is an element of $\mathbf{S}^{m}(B)$.

Definition 8.4. Let $\mathcal{D}$ be an ultrafilter on ${ }^{m} A$. (We sometimes write $m=\mathbf{m}(\mathcal{D})$ for this arity.)

(1) We say that the infinite indiscernible sequence $\bar{b}=\left\langle\bar{b}_{s}: s \in I\right\rangle$ is based on $\mathcal{D}$ when

$$
\left.\operatorname{tp}\left(\bar{b}_{s}, A+\overline{\mathbf{b}}_{>s}\right)=\operatorname{Av}\left(D, A+\overline{\mathbf{b}}_{>s}\right)\right)
$$

where $\overline{\mathbf{b}}_{>s}=\bigcup\left\{\bar{b}_{t}: t \in I, s<_{I} t\right\}$.

(2) For each $\mathcal{D}$, let $\mathrm{ob}(\mathcal{D})=\mathrm{ob}(\mathcal{D}, A)$ be the set of such $\overline{\mathbf{b}}$, i.e. the set of all infinite indiscernible sequences based on $\mathcal{D}$ (assuming the monster model $\mathfrak{C}$ is well defined).

In Definition 8.4 the elements approach $A$; of course we could have inverted the order. Given such an ultrafilter $\mathcal{D}$ and an infinte indiscernible sequence built from it, we may naturally ask when a given formula instantiated along this sequence is consistent.

Definition 8.5. Let $A \subseteq \mathfrak{C}$ and $\varphi=\varphi(\bar{x}, \bar{y})=\varphi(\bar{x}, \bar{y}, \bar{c})$ for $\bar{c} \in \mathfrak{C}$. ${ }^{18}$

(1) Let $\operatorname{uf}_{\varphi}(A)$ be the set of ultrafilters $\mathcal{D}$ on ${ }^{\lg (\bar{y})} A$ such that if $\overline{\mathbf{b}}=\left\langle\bar{b}_{s}: s \in I\right\rangle \in \mathrm{ob}(\mathcal{D})$, then

$$
\left\{\varphi\left(\bar{x}, \bar{b}_{s}, \bar{c}\right): s \in I\right\}
$$

is a consistent partial type.

\footnotetext{
${ }^{18}$ Usually, $\bar{c}$ is empty, and in any case, we can just incorporate it into the parameters $\bar{y}$.
} 
(2) For each $k<\omega$, let $\mathrm{uf}_{\varphi, k}(A)$ be the set of ultrafilters $\mathcal{D}$ on ${ }^{\lg (y)} A$ such that if $\overline{\mathbf{b}}=\left\langle\bar{b}_{s}: s \in I\right\rangle \in \mathrm{ob}(\mathcal{D})$ and $s_{0}<_{I} \cdots<_{I} s_{k-1}$, then

$$
\left\{\varphi\left(\bar{x}, \bar{b}_{s_{\ell}}, \bar{c}\right): \ell<k\right\}
$$

is a consistent partial type. So when $k=\infty$, we may omit it.

We arrive at a key definition of the section: higher formulas $(\varphi, A, \mathcal{D})$, triples such that $\varphi$ is indeed consistent when instantiated along any $\bar{b} \in \operatorname{ob}(\mathcal{D}, A)$. Two subsequent theorems of the section will characterize $\mathrm{SOP}_{2}$ and $\mathrm{SOP}_{3}$ in terms of the interaction of these higher formulas.

Definition 8.6 (Higher formulas). Let $\bar{m}=\left(m_{0}, m_{1}\right)$. Writing $m$ instead of $\bar{m}$ means $m_{0}=1$ and $m=m_{1}$, or that $m_{0}$ is clear from the context. Let $\mathbf{H F}_{k}^{\bar{m}}$ be the set of triples $\rho=(\varphi, A, \mathcal{D})$ where $\varphi=\varphi(\bar{x}, \bar{y})$ with $\ell(\bar{x})=m_{0}, \ell(\bar{y})=m_{1}$, and no more parameters, $\mathcal{D}$ is an ultrafilter on ${ }^{m_{1}} A$ and

$$
\mathcal{D} \in \operatorname{uf}_{\varphi, k}(A) .
$$

Definition 8.7. In the context of 8.6 .

(1) Let $\mathbf{H F}^{\bar{m}}=\mathbf{H F}_{\infty}^{\bar{m}}=\bigcap_{k} \mathbf{H F}_{k}^{\bar{m}}$.

(2) We may wish to consider higher formulas over a fixed set A, or a using a fixed formula $\varphi$, in which case our notation will be:

(a) Given $A$, we may write $\mathbf{H F}_{k}^{\bar{m}}(A)$, or " $(\varphi, \mathcal{D}) \in \mathbf{H F}_{k}^{\bar{m}}(A)$ ".

(b) $\operatorname{Let} \mathbf{H F}^{\bar{m}}(A)=\mathbf{H F}_{\infty}^{\bar{m}}(A)=\bigcap_{k} \mathbf{H F}_{k}^{\bar{m}}(A)$.

(c) Given $\varphi=\varphi(\bar{x}, \bar{y})$, with $\ell(\bar{x})=m_{0}$ and $\ell(\bar{y})=m_{1}$, we may write $\mathbf{H F}^{\varphi}=\mathbf{H F}_{\varphi}^{\bar{m}}, \mathbf{H F}_{\varphi, k}^{\bar{m}}(A)$, etc, where the subscript $\varphi$ means we restrict to triples whose first element is $\varphi$ with the given partition of variables.

(d) Call the elements of $\mathbf{H F}_{\varphi}(A)$ "higher $\varphi$-formulas over A."

Convention 8.8. In Defintion 8.6.(1), we may say "D is an ultrafilter over $A$ " without mentioning $m_{1}$ when it is clear from context.

We would like to study pairwise consistency or inconsistency of higher formulas as follows. Suppose we are given $\left(\varphi_{0}, A_{0}, \mathcal{D}_{0}\right),\left(\varphi_{1}, A_{1}, \mathcal{D}_{1}\right),\left\langle b_{0, s}: s \in I_{0}\right\rangle \in \mathrm{ob}\left(A_{0}, \mathcal{D}_{0}\right)$ and $\left\langle b_{0, t}: t \in I_{1}\right\rangle \in \mathrm{ob}\left(A_{1}, \mathcal{D}_{1}\right)$. If we choose $s \in I_{0}$ and $t \in I_{1}$, will $\varphi_{0}\left(\bar{x}, \bar{b}_{0, s}\right)$ and $\varphi_{1}\left(\bar{x}, \bar{b}_{1, t}\right)$ be consistent? What if we choose finitely many instances from each list? The specter of $S O P_{3}$ suggests that we should first fix an interpolation of $I_{0}$ and $I_{1}$ into a single linear order $I$ and pay attention to the relative position of the indices $s$ and $t$. The notation we now introduce in 8.98 .10 is one way to handle this (most of the time we use $\sigma=2$ ).

Definition 8.9. (Partitions of linear orders, [Sh:950] Definition 1.39)

Let $K_{p, \sigma}$ be the class of triples

$$
\left(I,<_{I},\left(P_{i}^{I}\right)_{i<\sigma}\right)
$$

where $I$ is linearly ordered by $<_{I}$ and $\left\langle P_{i}^{I}: i<\sigma\right\rangle$ is a partition of $I$. 
Definition 8.10. Fix $\sigma$ and suppose $\left\langle\left(\varphi_{i}, A_{i}, D_{i}\right): i<\sigma\right\rangle$ is a sequence of higher formulas. Let $\bar{A}=\left\langle A_{i}: i<\sigma\right\rangle$ and let $\bar{D}=\left\langle D_{i}: i<\sigma\right\rangle$. Define

$$
\mathrm{ob}(\bar{D})=\mathrm{ob}(\bar{D}, \bar{A})
$$

to be the set of $\overline{\mathbf{b}}=\left\langle\bar{b}_{s}: s \in I\right\rangle$ such that:

(1) $I \in K_{p, \sigma}$

(2) if $s \in P_{i}^{I}$ then:

- $\ell g\left(\bar{b}_{s}\right)=\mathbf{m}\left(D_{i}\right)$

- $\operatorname{tp}\left(\bar{b}_{s}, \bigcup_{j<\sigma} A_{j} \cup \overline{\mathbf{b}}_{>s}\right) \subseteq \operatorname{Av}\left(D_{i}\right):=\operatorname{Av}\left(D_{i}, \mathfrak{C}_{\tau}\right)$.

We may write $A$ instead of $\bar{A}$ when all the $A_{i}$ are the same. We may write $i=$ $\mathbf{i}(s)=\mathbf{i}(s, I)$ and we may write $I_{\overline{\mathbf{b}}}=I[\overline{\mathbf{b}}]$ for $I$.

Note that in Definition 8.10 , we do not require that $\overline{\mathbf{b}}$ be indiscernible; in fact, it may consist of sequences of differing lengths, if the $\mathbf{m}\left(D_{i}\right)$ differ.

Definition 8.11 ( $n$-inconsistent higher formulas). Assume that for $\ell=0,1$,

$$
\rho_{\ell}=\left(\varphi_{\ell}, A_{\ell}, D_{\ell}\right) \in \mathbf{H F}_{k_{\ell}}^{\bar{m}_{\ell}} .
$$

(1) We say $\left(\varphi_{0}, A_{0}, D_{0}\right)$ is n-contradictory to $\left(\varphi_{1}, A_{1}, D_{1}\right)$ when:

for every $\overline{\mathbf{b}} \in \mathrm{ob}\left(\left\langle D_{0}, D_{1}\right\rangle,\left\langle A_{0}, A_{1}\right\rangle\right)$ and every $s_{0}<\ldots<s_{2 n-1}$ with $s_{\ell} \in P_{0}^{I}[\overline{\mathbf{b}}]$ for $\ell<n$ and $s_{\ell} \in P_{1}^{I}[\overline{\mathbf{b}}]$ for $\ell \in[n, 2 n)$, we have that

$$
\left\{\varphi_{0}\left(\bar{x}, \bar{b}_{s_{\ell}}\right): \ell<n\right\} \cup\left\{\varphi_{1}\left(\bar{x}, \bar{b}_{s_{\ell}}\right): \ell \in[n, 2 n)\right\}
$$

is contradictory.

(2) In " $n$-contradictory," if $n=1$ we may omit it and writing $n=\infty$ means "for some $n "$. Of course, " $n$-consistent" is the negation.

(3) We say that $\left(\varphi_{0}, A_{0}, D_{0}\right)$ and $\left(\varphi_{1}, A_{1}, D_{1}\right)$ are mutually $n$-contradictory when $\left(\varphi_{\ell}, A_{\ell}, D_{\ell}\right)$ is n-contradictory to $\left(\varphi_{1-\ell}, A_{1-\ell}, D_{1-\ell}\right)$ for $\ell=0,1$. On the symmetry of this notion, see Lemma 8.15 below.

(4) "The set of $\left\{\rho_{i}=\left(\varphi_{i}, A_{i}, D_{i}\right): i \in S\right\}$ are pairwise $n$-contradictory" will mean that each pair is mutually $n$-contradictory.

\section{Discussion 8.12.}

(1) In 8.11(1) we could have allowed the choice of elements from the two partitions to alternate. However, we will see this is immaterial for $\mathrm{NSOP}_{3}$, and moreover is a little less natural when replacing $n$-contradictory higher formulas by 1-contradictory derived formulas arising as $n$-fold conjunctions.

(2) This definition enforces an order between the elements $s_{i}$ in different partitions; one could give a different definition requiring only that $s_{0}<\cdots<$ $s_{n-1}$ and $s_{n}<\cdots<s_{2 n-1}$.

Definition 8.13. Say that $T$ has symmetric inconsistency if, fixing $m=\ell(\bar{x})$, for any $y^{19} m_{1}, m_{2}<\omega, \varphi_{\ell}=\varphi_{\ell}\left(\bar{x}_{[m]}, \bar{y}_{\left[m_{\ell}\right]}\right)$ and $\left(\varphi_{\ell}, A_{\ell}, \mathcal{D}_{\ell}\right) \in \mathbf{H F}^{m_{\ell}}$ for $\ell=1,2$, we have that $\left(\varphi_{1}, A_{1}, \mathcal{D}_{1}\right)$ is $\infty$-contradictory to $\left(\varphi_{2}, A_{2}, \mathcal{D}_{2}\right)$ iff $\left(\varphi_{2}, A_{2}, \mathcal{D}_{2}\right)$ is $\infty$ contradictory to $\left(\varphi_{1}, A_{1}, \mathcal{D}_{1}\right)$.

\footnotetext{
${ }^{19}$ The notation $\varphi_{\ell}\left(\bar{x}_{[m]}, \bar{y}_{\left[m_{\ell}\right]}\right)$ means that $\ell(\bar{x})=m$ and $\ell(\bar{y})=m_{\ell}$.
} 
As desired, this definition picks up on the asymmetry of $\mathrm{SOP}_{3}$ :

Claim 8.14. If $T$ has $S_{3} P_{3}$, then 1-inconsistency is not symmetric, i.e. T has symmetric inconsistency in the sense of 8.13 .

Proof. Let the sequence $\left\langle\bar{a}_{n}: n<\omega\right\rangle$ and the formulas $\varphi(\bar{x}, \bar{y}), \psi(\bar{x}, \bar{y})$ witness $S O P_{3}$, see 8.2 . Let $A=\left\{\bar{a}_{n}: n<\omega\right\}$. Let $\mathcal{D}$ be an ultrafilter on ${ }^{\lg (\bar{y})} A$ such that $\left\{\bar{a}_{n}: n>k\right\} \in \mathcal{D}$ for every $k<\omega$. Let $\rho_{1}=(\varphi, A, \mathcal{D}), \rho_{2}=(\psi, A, \mathcal{D})$. To see that $\rho_{2}$ is 1 -consistent with $\rho_{1}$ but $\rho_{1}$ is not 1 -consistent with $\rho_{2}$, let $I$ be the linear order $\omega+\omega^{*}$. Let $\left(P_{0}, P_{1}\right)$ be any partition of $I$ into two infinite sets, and let $\left\langle\bar{b}_{s}: s \in I\right\rangle$ be as in 8.10 for $((\mathcal{D}, \mathcal{D}),(A, A))$.

Lemma 8.15 (Symmetry lemma). For $T$ complete the following are equivalent.

(1) $\mathrm{T}$ is $\mathrm{SOP}_{3}$.

(2) $T$ has symmetric inconsistency.

Proof. The direction (1) implies (2) is given by Claim 8.14 .

For the other direction, we will show how an instance of symmetric inconsistency gives rise to $S O P_{3}$. For notational simplicity, we use $m=\ell(\bar{x})=1$, since for $S O P_{3}$ the arity does not matter. Suppose we are given $m_{0}, m_{1}<\omega$ and $\left(\varphi_{\ell}, A_{\ell}, \mathcal{D}_{\ell}\right) \in$ $\mathbf{H F}^{m_{\ell}}$ for $\ell=0,1$.

Let $A=A_{0} \cup A_{1}, m=m_{0}+m_{1}$ and

$D=D_{0} \times D_{1}=\left\{X \subseteq{ }^{m} A:\left\{\bar{a}_{0} \in{ }^{m_{0}} A_{0}:\left\{\bar{a}_{1} \in{ }^{m_{1}}\left(A_{1}\right): \bar{a}_{0} \frown \bar{a}_{1} \in X\right\} \in D_{1}\right\} \in D_{0}\right\}$.

By construction, $D$ is an ultrafilter on ${ }^{m} A$. Suppose $I$ is a linear order and

$$
\overline{\mathbf{a}}=\left\langle\bar{a}_{s}=\bar{a}_{0, s} \frown \bar{a}_{1, s}: s \in I\right\rangle
$$

is an indiscernible sequence based on $D$, with $\ell\left(a_{\ell, s}\right)=m_{\ell}$, thus $\left\langle\bar{a}_{\ell, s}: s \in I\right\rangle$ is also an indiscernible sequence based on $D_{\ell}$ for $\ell=0,1$. For any $h: I \rightarrow\{0,1\}$, we may consider the partition given by

$$
I_{h}=\left(I,<_{I},\left(P_{i}^{h}\right)_{i<2}\right) \text { where } P_{i}^{h}=\{s: h(s)=i\}
$$

Then $I_{h} \in K_{p,\{0,1\}}$ and $\overline{\mathbf{a}}_{h}:=\left\langle a_{h(s), s}: s \in I\right\rangle$ is $I_{h}$-indiscernible based on $\left(D_{0}, D_{1}\right)$.

FIGURE 1. A sample partition of $I$. $I$ indexes a sequence of $m_{0}+m_{1}$-tuples which is indiscernible based on $D$. Thus, restricting to the first $m_{0}$ elements of tuples with indices in the black regions gives an indiscernible sequence based on $D_{0}$, whereas restricting to the last $m_{1}$ elements of tuples with indices in the grey regions gives an indiscernible sequence based on $D_{1}$.

Moreover, if $\left\langle s_{\alpha}: \alpha<\omega+\omega\right\rangle$ is $<_{I}$-increasing, then:

$(1)_{n}$ the following are equivalent:

(a) $\left\{\varphi_{0}\left(x, \bar{a}_{0, s_{\alpha}}\right): \alpha<n\right\} \cup\left\{\varphi_{1}\left(x, \bar{a}_{1, s_{\omega+\alpha}}\right): \alpha<n\right\}$ is contradictory

(b) $\left(\varphi_{0}, A_{0}, D_{0}\right)$ is $n$-contradictory to $\left(\varphi_{1}, A_{1}, D_{1}\right)$

$(2)_{n}$ the following are equivalent: 
FIGURE 2. Let the image indicate that we instantiate $\varphi_{0} n$ times along the $D_{0}$-indiscernible sequence indexed by the black region and $\varphi_{1} n$ times along the $D_{1}$-indiscernible sequence indexed by the grey region.

(d) $\left\{\varphi_{1}\left(x, \bar{a}_{1, s_{\alpha}}\right): \alpha<n\right\} \cup\left\{\varphi_{0}\left(x, \bar{a}_{0, s_{\omega+\alpha}}\right): \alpha<n\right\}$ is contradictory

(e) $\left(\varphi_{1}, A_{1}, D_{1}\right)$ is $n$-contradictory to $\left(\varphi_{0}, A_{0}, D_{0}\right)$

FIGURE 3. Let the image indicate that we instantiate $\varphi_{1} n$ times along the $D_{1}$-indiscernible sequence indexed by the grey region and $\varphi_{0} n$ times along the $D_{0}$-indiscernible sequence indexed by the black region. Then for any given $n$, a disparity in consistency between Figures 2 and 3 leads, by taking conjunctions, to an instance of $\mathrm{SOP}_{3}$.

Let $\bar{y}=\bar{y}_{0}^{\frown} \bar{y}_{1}, \varphi_{0}^{+}=\varphi_{0}^{+}(x, \bar{y})=\varphi_{0}^{+}\left(x, \bar{y}_{0}, \bar{y}_{1}\right)=\varphi_{0}\left(x, \bar{y}_{0}\right)$, and $\varphi_{1}^{+}=\varphi_{1}^{+}(x, \bar{y})=$ $\varphi_{1}^{+}\left(x, \bar{y}_{0}, \bar{y}_{1}\right)=\varphi_{1}\left(x, \bar{y}_{1}\right)$, i.e. these are the given formulas formally considered as having more variables. Then for each $n$, to $(1)_{n}$ above, we may add the equivalent condition:

(c) $)_{n}\left\{\varphi_{0}^{+}\left(x, \bar{a}_{s_{\alpha}}\right): \alpha<n\right\} \cup\left\{\varphi_{1}^{+}\left(x, \bar{a}_{s_{\omega+\alpha}}\right): \alpha<n\right\}$ is contradictory

Likewise, for each $n$, to $(2)_{n}$ above, we may add the equivalent condition:

(f) $)_{n}\left\{\varphi_{1}^{+}\left(x, \bar{a}_{s_{\alpha}}\right): \alpha<n\right\} \cup\left\{\varphi_{0}^{+}\left(x, \bar{a}_{s_{\omega+\alpha}}\right): \alpha<n\right\}$ is contradictory

Now if it is not the case that for all $n(\mathrm{c})_{n}$ iff for all $n(\mathrm{f})_{n}$, we have a witness to $S O P_{3}$ for $T$ (given by the conjunctions of $n$ copies of $\varphi_{0}$ and of $\varphi_{1}$, respectively). This completes the proof.

As a corollary of the proof of Lemma 8.15 we have:

Corollary 8.16. Assume $T$ is $N S O P_{3}$. If $p_{\ell}=\left(\varphi_{\ell}, A_{\ell}, \mathcal{D}_{\ell}\right)$ for $\ell=0,1$ and $p_{0}$ is $n$-consistent with $p_{1}$ for every $n$ (i.e., not $\infty$-contradictory), then if $I \in K_{\sigma}^{P}, \sigma=2$ and we have $\left\langle\bar{b}_{s}: s \in I\right\rangle$ as usual, then $\left\{\varphi_{\ell}\left(x, \bar{b}_{s}\right): s \in P_{\ell}^{I}, \ell<2\right\}$ is consistent.

We now work towards Theorem 8.21, using higher formulas to characterize $\mathrm{NSOP}_{2}$. First, we show that having $\mathrm{SOP}_{2}$ means many pairwise 1-contradictory higher formulas. Recall that:

Definition 8.17. $\operatorname{Ded}^{+}(\lambda)=\sup \left\{|\lim (\mathcal{T})|^{+}: \mathcal{T} \subseteq{ }^{\lambda>} 2\right.$ is nonempty, closed under initial segments and has no $\triangleleft$-maximal members and has cardinality $\leq \lambda\}$.

Claim 8.18. Assume $\varphi(\bar{x}, \bar{y})$ has $\mathrm{SOP}_{2}$ in $T$ and $\lambda<\mu<\operatorname{Ded}(\lambda)$. Then there is $A \subseteq \mathfrak{C}_{T}$ of cardinality $\lambda$ and $D_{\alpha} \in \mathrm{uf}_{\varphi}(A)$ for $\alpha<\mu$ such that $\left\langle\left(\varphi, D_{\alpha}\right): \alpha<\mu\right)$ are pairwise 1-contradictory.

Proof. Let the tree $\mathcal{T}$ witness that $\mu<\operatorname{Ded}(\lambda)$ and let $\nu_{\alpha} \in \lim (\mathcal{T})$ (i.e. the "leaves") for $\alpha<\mu$ be pairwise distinct. Let $\left\langle\bar{a}_{\eta}: \eta \in \mathcal{T}\right\rangle$ be such that: 
(a) $\left(\varphi\left(\bar{x}, \bar{a}_{\eta}\right), \varphi\left(\bar{x}, \bar{a}_{\nu}\right)\right.$ are contradictory when $\eta \perp \nu$ are from $\mathcal{T}$

(b) $\left\{\varphi\left(x, \bar{a}_{\eta\lceil\alpha}\right): \alpha \leq \ell g(\eta)\right\}$ is a consistent partial type for $\eta \in \mathcal{T}$.

Let $A=\cup\left\{\bar{a}_{\eta}: \eta \in \mathcal{T}\right\}$ and for $\alpha<\mu$ let $D_{\alpha}$ be an ultrafilter on ${ }^{\ell g(\bar{y})} A$ concentrating on the branch $\nu_{\alpha}$, i.e. such that:

$$
\text { if } \beta<\ell g\left(\nu_{\alpha}\right), \alpha<\mu \text { then }\left\{\bar{a}_{\rho}: \nu_{\alpha} \uparrow \beta \unlhd \rho \triangleleft \nu_{\alpha}\right\} \in D_{\alpha}
$$

Clearly these ultrafilters are as desired.

We will need notation for finitary approximations to $S O P_{2}$-trees.

Definition 8.19. We say that $(T, \varphi)$ has $\operatorname{NSOP}_{2, n}$ when there are no $\bar{b}_{\eta} \in{ }^{\ell g(\bar{y})} \mathfrak{C}$ for $\eta \in{ }^{n>2}$ such that

(1) $\eta \perp \nu \Rightarrow \varphi\left(\bar{x}, \bar{b}_{\eta}\right), \varphi\left(\bar{x}, \bar{b}_{\nu}\right)$ are incompatible

(2) for $\eta \in{ }^{n} 2,\left\{\varphi\left(\bar{x}, \bar{b}_{\eta \uparrow \ell}\right): \ell<n\right\}$ is a type.

Fact 8.20. $(T, \varphi) \models N S O P_{2}$ iff $\bigvee_{n}\left((T, \varphi) \models N S O P_{2, n}\right)$.

We now arrive at the second theorem of the section, which shows how from many pairwise contradictory higher formulas we may build an $S O P_{2}$-tree, complementing 8.18. Recall that by the Symmetry Lemma 8.15 above, as $N S O P_{2}$ implies $N S O P_{3}$, being contradictory is a symmetric notion.

Theorem 8.21. For a theory $T$ the following are equivalent:

(1) For every infinite $A$ and formula $\varphi$, there are no more than $|A|$ pairwise 1-contradictory higher $\varphi$-formulas over $A$.

(2) $\mathrm{T}$ has $\mathrm{NSOP}_{2}$.

Proof. In Claim 8.18, it was shown that $S O P_{2}$ implies many pairwise 1-contradictory higher formulas. So it remains to prove the other direction: many pairwise 1contradictory higher formulas imply $\mathrm{SOP}_{2}$.

Step 0: Setup. Let $\lambda=|A|^{+}$and by Fact 8.20 , let $n$ be such that $(T, \varphi)$ has $N S O P_{2, n}$.

Assume for a contradiction that:

(1) $\left(\varphi, D_{\alpha}\right) \in \mathbf{H F}_{\varphi}^{m}(A)$ for $\alpha<\lambda$ are pairwise 1-contradictory ${ }^{20}$ and

(2) fixing some infinite linear order $I$, let $\overline{\mathbf{b}}_{\alpha}=\left\langle b_{\alpha, s}: s \in I\right\rangle \in \mathrm{ob}\left(D_{\alpha}\right)$ for $\alpha<\lambda$.

(Recall that the definition of 1-contradictory is for any such $\overline{\mathbf{b}}$.) We will use just that $\lambda=\operatorname{cf}(\lambda)>|A| \geq \aleph_{0}$.

Step 1: Approximations. We define the set AP of approximations (to a full $S O P_{2, n^{-}}$ tree) to be the set of $\mathbf{x}$ consisting of: 21

(1.1) $\Lambda \subseteq n>2, \bar{a}$ where:

\footnotetext{
${ }^{20}$ Here $\mathbf{H F}_{\varphi, n}^{m}(A)$ suffices.

${ }^{21}$ Alternately, we could consider: in $\Lambda_{x}, \operatorname{frt}(\mathbf{x})$ is a set of pairwise incomparable elements, e.g. the $\triangleleft$-maximal $\eta \in \Lambda$ of length $<n-1$. This is simpler here, but then the induction step would require two steps: add $\eta^{\frown}\langle 0\rangle$ or $\eta^{\frown}\langle 1\rangle$ for some $\eta \in \operatorname{frt}(\mathbf{x})$.
} 
(a) $\Lambda$ is non-empty downward closed

(b) $\bar{a}=\left\langle\bar{a}_{\eta}: \eta \in \Lambda\right\rangle$ with each $\bar{a}_{\eta} \in{ }^{\lg (\bar{y})} A$

(c) if $\eta \perp \nu$ are from $\Lambda$ then $\varphi\left(\bar{x}, \bar{a}_{\eta}\right), \varphi\left(\bar{x}, \bar{a}_{\nu}\right)$ are incompatible

(d) if $\eta \in \Lambda$ then $\left\{\varphi\left(\bar{x}, \bar{a}_{\eta\lceil\ell}\right): \ell \leq \ell g(\eta)\right\}$ is a consistent partial type

(e) if $\nu^{\curlyvee}\langle 1\rangle \in \Lambda$ then $\nu^{\curlyvee}\langle 0\rangle \in \Lambda$.

(1.2) $\overline{\mathcal{U}}=\left\langle\mathcal{U}_{\eta}: \eta \in \operatorname{frt}(\Lambda)\right\rangle$, where:

(a) $\operatorname{frt}(\Lambda):=\left\{\eta \in \Lambda: \ell g(\eta)<n-1\right.$ and $\eta^{\wedge}\langle 0\rangle \notin \Lambda$ or $\left.\eta^{\wedge}\langle 1\rangle \notin \Lambda\right\}$

(the "frontier" for our inductive construction of a tree, i.e., the nodes without two immediate successors)

(b) each $\mathcal{U}_{\eta} \subseteq \lambda$ has cardinality $\lambda$

(the intention is a set of indices for the $\overline{\mathbf{b}}_{\alpha}$ from Step 0)

(c) $\mathcal{U}_{\eta} \cap \mathcal{U}_{\nu}=\emptyset$ for $\eta \neq \nu$

(d) if $\eta \unlhd \nu$ are from $\operatorname{frt}(\Lambda), k<\omega, s_{0}, \ldots, s_{k-1} \in I, k+\ell g(\eta) \leq n$ and $\alpha \in \mathcal{U}_{\nu}$ then

$$
\left\{\varphi\left(x, \bar{a}_{\eta \nmid \ell}\right): \ell \leq \ell g(\eta)\right\} \cup\left\{\varphi\left(x, \bar{b}_{\alpha, s_{\ell}}\right): \ell<k\right\}
$$

is a consistent partial type.

(e) if $\eta \in \operatorname{frt}(\mathbf{x}), s \in I, \alpha \in \mathcal{U}_{\eta}$ and $\nu \in \Lambda, \neg(\nu \unlhd \eta)$, then $\varphi\left(\bar{x}, \bar{b}_{\alpha, s}\right), \varphi\left(\bar{x}, a_{\nu}\right)$ are incompatible.

Note the role of the two kinds of parameters: the $\bar{b}_{\alpha, s}$ from Step 0, and the parameters $\bar{a}_{\eta}$ for the tree. Informally, the $\mathcal{U}_{\eta}$ tell us in which sequences we can expect to continue our consistent partial type while maintaining inconsistency elsewhere.

We define a two-place relation $\leq_{\mathrm{AP}}$ on $\mathrm{AP}$ in the natural way: $\mathbf{x} \leq_{\mathrm{AP}} \mathbf{y}$ iff

(a) $\mathbf{x}, \mathbf{y} \in \mathrm{AP}$

(b) $\Lambda_{\mathbf{x}} \subseteq \Lambda_{\mathbf{y}}$

(c) $\bar{a}_{\mathbf{x}, \eta}=\bar{a}_{\mathbf{y}, \eta}$ for $\eta \in \Lambda_{\mathbf{x}}$

(d) if $\eta \in \operatorname{frt}\left(\Lambda_{\mathbf{x}}\right) \cap \operatorname{frt}\left(\Lambda_{\mathbf{y}}\right)$ then $\mathcal{U}_{\mathbf{y}, \eta} \subseteq \mathcal{U}_{\mathbf{x}, \eta}$

(e) if $\eta \in \operatorname{frt}\left(\Lambda_{\mathbf{x}}\right), \nu \in \operatorname{frt}\left(\Lambda_{\mathbf{y}}\right)$ and $\eta \triangleleft \nu$ but $\nu \uparrow(\ell g(\eta)+1) \notin \Lambda_{\mathbf{x}}$, then $\mathcal{U}_{\mathbf{y}, \nu} \subseteq \mathcal{U}_{\mathbf{x}, \eta}$.

Step 2: Strategy. Clearly $\leq_{\mathrm{AP}}$ is a partial order on AP. By choice of $n$ in Step 0, if $\mathbf{x} \in \mathrm{AP}$ then $\Lambda \subsetneq^{n>} 2$. Thus, to obtain a contradiction (and complete the proof) it will suffice to show that:

(2.1) there is $\mathbf{z} \in \mathrm{AP}$ with $\Lambda_{\mathbf{z}}=\{<>\}$, i.e. $\mathrm{AP} \neq \emptyset$.

(2.2) if $\mathbf{x} \in \mathrm{AP}$ then there is $\mathbf{y} \in \mathrm{AP}$ such that $\left|\Lambda_{\mathbf{x}}\right|<\left|\Lambda_{\mathbf{y}}\right|$, in fact, $\mathbf{x}<_{\mathrm{AP}} \mathbf{y}$.

Step 3: Verifying Condition (2.1). Let $s_{0}<_{I} \ldots<_{I} s_{n}$. For each $\alpha<\lambda$ clearly $\mathfrak{C} \models(\exists \bar{x}) \bigwedge_{\ell \leq n} \varphi\left(\bar{x}, \bar{b}_{\alpha, s_{\ell}}\right)$. So by (1) and (2) of Step 0 ,

$$
\mathcal{X}_{\alpha}=\left\{\bar{a} \in{ }^{\ell g(\bar{y})} A: \mathfrak{C} \models(\exists \bar{x})\left[\varphi(\bar{x}, \bar{a}) \wedge \bigwedge_{\ell=1}^{n} \varphi\left(x, \bar{b}_{\alpha, s_{\ell}}\right)\right]\right\} \in D_{\alpha} .
$$


For each $\alpha<\lambda$, choose $\bar{b}_{\alpha} \in \mathcal{X}_{\alpha}$. [So $\bar{b}_{\alpha}$ is "canonically consistent" with the partial $\varphi$-type given along the sequence $\overline{\mathbf{b}}_{\alpha}$ by any $n$ members.] As $|A|<\lambda$, the set ${ }^{\ell g(\bar{y})} A$ has cardinality $<\lambda=\operatorname{cf}(\lambda)$ so for some $\bar{b}$ the set

$$
\mathcal{U}=\left\{\alpha<\lambda: \bar{b}_{\alpha}=\bar{b}\right\}
$$

has cardinality $\lambda$.

Now define $\mathbf{z}$ by:

- $\Lambda_{\mathbf{z}}=\{<>\}$

- $\bar{a}_{<>}=\bar{b}$

- $\mathcal{U}_{<>}=\mathcal{U}$.

and $\mathbf{z}$ is as required.

Step 4: Proving Condition (2.2). Let $\Lambda=\Lambda_{\mathbf{x}}$.

The situation at the inductive step is essentially as follows. We would like the tree to become full, so we choose $\varrho$ which is minimal for the property of not having

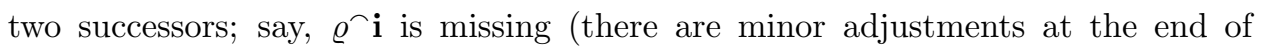
this step depending on whether it has a successor at 0 or 1 ). We try to find a corresponding $\bar{a}_{\varrho^{-} \mathbf{i}}$ for the missing successor of this node, and its set of compatible indices $\mathcal{U}_{\varrho^{-} \mathbf{i}}$, subject to the following constraints:

(i) consistency of $\bar{a}_{\varrho}>\mathbf{i}$ with comparable nodes, $(1.1)(\mathrm{d})$

(ii) inconsistency of $\bar{a}_{\varrho^{-} \mathbf{i}}$ with incomparable nodes, (1.1)(c)

(iii) consistency of $\bar{a}_{\varrho}-\mathbf{i}$ with large subsets of $\mathcal{U}_{\nu}$, for compatible $\nu$ for $(1.2)(\mathrm{d})$

(iv) inconsistency of $\bar{a}_{\varrho}-\mathbf{i}$ with $\mathcal{U}_{\eta}$, for incompatible $\eta$, for $(1.2)(\mathrm{e})$

(v) disjointness of $\mathcal{U}_{\varrho^{-} \mathbf{i}}$ from large subsets of $\mathcal{U}_{\nu}$, for incompatible $\nu$, for $(1.2)(\mathrm{c})$

In this informal explanation, "large" stands in for the fact that we will also have to refine the other $\mathcal{U}_{\eta}$ to get actual inconsistency or an actual empty intersection. This completes the description of intent.

Now to begin, choose $\varrho \in \operatorname{frt}(\Lambda)$ be of minimal length, hence necessarily $\ell g(\varrho)<$ $n-1$ and we can choose $\iota<2$ such that $\varrho^{\wedge}\langle\iota\rangle \notin \Lambda$. Let $k=n-\lg (\varrho)$.

Fix for a while a choice of a distinguished element of $\mathcal{U}_{\eta}$ for each $\eta \in \operatorname{frt}(\Lambda)$, that is, $\bar{\alpha}=\left\langle\alpha_{\eta}: \eta \in \operatorname{frt}(\Lambda)\right\rangle$ where $\alpha_{\eta} \in \mathcal{U}_{\eta}$, and let $s_{0}<_{I} \ldots<_{I} s_{k-1}$.

By our choice of $\varrho$ and $(1.2)(\mathrm{c})$, for every ${ }^{22} \eta \in \operatorname{frt}\left(\Lambda_{\mathbf{x}}\right) \backslash\{\varrho\}$ we have that $\alpha_{\eta} \neq \alpha_{\rho}$, hence the following set belongs to $D_{\alpha_{\varrho}}$ :

$$
\begin{aligned}
& \mathcal{X}_{\bar{\alpha}, \eta}=\mathcal{X}_{\bar{\alpha}, \eta}^{1} \cup \mathcal{X}_{\bar{\alpha}, \eta}^{2} \text { where } \\
& \mathcal{X}_{\bar{\alpha}, \eta}^{1}=\left\{\bar{b} \in{ }^{\lg (\bar{y})} A:: \varphi(\bar{x}, \bar{b}), \varphi\left(\bar{x}, \bar{a}_{\nu}\right) \text { are incompatible, where } \nu \in \Lambda \wedge \neg(\nu \unlhd \rho)\right\} \\
& \mathcal{X}_{\bar{\alpha}, \eta}^{2}=\left\{\bar{b} \in{ }^{\ell g(\bar{y})} A: \varphi(\bar{x}, \bar{b}), \varphi\left(\bar{x}, \bar{b}_{\alpha_{\eta}, s_{0}}\right) \text { are incompatible }\right\}
\end{aligned}
$$

since the $\left(\varphi, \mathcal{D}_{\alpha}\right)$ are pairwise 1-contradictory and as $\left\langle\alpha_{\ell}: \ell \in \operatorname{frt}(\mathbf{x})\right\rangle$ is without repetitions by the assumption $(1.2)(\mathrm{c})$ for $\mathcal{X}_{\bar{\alpha}, \eta}^{2}$ and (1.2)(e) for $\mathcal{X}_{\bar{\alpha}, \eta}^{1}$.

\footnotetext{
${ }^{22}$ If $\rho$ has precisely one successor in the tree, this successor may be from $\operatorname{frt}\left(\Lambda_{\mathbf{x}}\right)$.
} 
Furthermore, as $(1.2)(\mathrm{d})$ holds for $\mathbf{x}$, the following set belongs to $D_{\alpha_{\varrho}}$ :

$$
\begin{aligned}
\mathcal{Z}_{\bar{\alpha}, \varrho}:=\left\{\bar{b} \in{ }^{\ell g(\bar{y})} A:\right. & \left\{\varphi\left(\bar{x}, \bar{a}_{\eta \uparrow \ell}\right): \ell \leq \ell g(\varrho)\right\} \\
& \cup\{\varphi(\bar{x}, \bar{b})\} \\
& \left.\cup\left\{\varphi\left(\bar{x}, \bar{b}_{\alpha_{\varrho}, s_{\ell}}\right): \ell<n-\ell g(\varrho)-1\right\} \text { is a type }\right\}
\end{aligned}
$$

Let $\mathcal{X}_{\bar{\alpha}}=\cap\left\{\mathcal{X}_{\bar{\alpha}, \eta}: \eta \in \operatorname{frt}(\Lambda) \backslash\{\varrho\}\right.$ and not comparable to $\left.\varrho\right\} \cap \mathcal{Z}_{\bar{\alpha}, \varrho}$. Since $\Lambda$ is finite and $D_{\alpha_{\varrho}}$ is a filter, $\mathcal{X}_{\bar{\alpha}} \in D_{\alpha_{\varrho}}$.

By the choice of our sequence of ultrafilters in Step 0, for every $\beta \in \mathcal{U}_{\mathbf{x}, \varrho} \backslash\left\{\alpha_{\varrho}\right\}$, we know that $\left(\varphi, D_{\alpha_{e}}\right),\left(\varphi, D_{\beta}\right)$ are 1 -contradictory. So

$$
\mathcal{Y}_{\bar{\alpha}, \beta}=\left\{\bar{b} \in{ }^{\ell g(\bar{y})} A: \varphi(x, \bar{b}), \varphi\left(x, \bar{b}_{\beta, s_{0}}\right) \text { are incompatible }\right\}
$$

belongs to $D_{\alpha_{\varrho}}$. So we may choose $\bar{b}_{\bar{\alpha}, \beta} \in{ }^{\ell g(\bar{y})} A$ which belongs to $\mathcal{X}_{\bar{\alpha}} \cap \mathcal{Y}_{\bar{\alpha}, \beta}$. As $\left|\mathcal{U}_{\mathbf{x}, \varrho}\right|=\lambda=\operatorname{cf}(\lambda)>|A|$, there is $\bar{b}_{\bar{\alpha}} \in{ }^{\ell g(\bar{y})} A$ such that

$$
\mathcal{W}_{\bar{\alpha}}=\left\{\beta \in \mathcal{U}_{\mathbf{x}, \varrho}: \bar{b}_{\bar{\alpha}, \beta}=\bar{b}_{\bar{\alpha}}\right\}
$$

has cardinality $\lambda$.

Recall that all of this is for a fixed $\bar{\alpha},\left\langle s_{0}, \ldots, s_{k-1}\right\rangle$.

Now continue to fix $s_{0}<_{I} \cdots<_{I} s_{k-1}$. For every $\gamma<\lambda$ we let $\bar{\alpha}_{\gamma}=\left\langle\alpha_{\gamma, \eta}: \eta \in\right.$ $\operatorname{frt}(\Lambda)\rangle$ be defined by

$$
\alpha_{\gamma, \eta}=\min \left(\mathcal{U}_{\mathbf{x}, \eta} \backslash \gamma\right)
$$

which is well defined as $\mathcal{U}_{\mathbf{x}, \eta}$ is an unbounded subset of $\lambda$. So for every $\gamma<\lambda$, we have that $\bar{\alpha}_{\gamma}, \bar{b}_{\bar{\alpha}_{\gamma}}, \mathcal{W}_{\bar{\alpha}_{\gamma}}$ are well defined. So for some $\bar{b}_{*}$ we have

$$
\mathcal{W}_{\bar{b}_{*}}=\left\{\gamma<\lambda: \bar{b}_{\bar{\alpha}_{\gamma}}=\bar{b}_{*}\right\}
$$

is an unbounded subset of $\lambda$.

We can now define the necessary objects, in two cases. For Case 1 below, the final definitions depend on whether the node we are dealing with has a sibling or not.

Case 1: $1=\left|\Lambda_{\mathbf{x}} \cap\left\{\varrho^{\wedge}\langle 0\rangle, \varrho^{\wedge}\langle 1\rangle\right\}\right|$

Let $\iota$ be such that $\varrho^{\wedge}\langle\iota\rangle \notin \Lambda_{\mathbf{x}}$. We define $\mathbf{y}$ as follows:

$\oplus(a) \quad \Lambda_{\mathbf{y}}=\Lambda_{\mathbf{x}} \cup\left\{\varrho^{\wedge}\langle\iota\rangle\right\}$, hence $\operatorname{frt}\left(\Lambda_{\mathbf{y}}\right)=\operatorname{frt}(\Lambda) \cup\left\{\varrho^{\wedge}\langle\iota\rangle\right\} \backslash\{\varrho\}$

(b) $\bar{b}_{\mathbf{y}, \eta}$ is $\bar{b}_{\mathbf{x}, \eta}$ if $\eta \in \Lambda_{\mathbf{x}}$

(c) $\bar{b}_{\mathbf{y}, \eta}$ is $\bar{b}_{*}$ if $\eta \in \varrho^{\wedge}\langle\iota\rangle$

(d) if $\eta \in \operatorname{frt}(\Lambda) \backslash\{\varrho\}$ then $\mathcal{U}_{\mathbf{y}, \eta}$ is $\left\{\alpha \in \mathcal{U}_{\mathbf{x}, \eta}\right.$ : for some $\gamma<\lambda, \bar{b}_{\bar{\alpha}_{\gamma}}=$ $\bar{b}_{*}$ and $\left.\alpha=\alpha_{\gamma, \eta}\right\}$

(e) if $\eta=\varrho^{\wedge}\langle\iota\rangle$ then $\mathcal{U}_{\mathbf{y}, \eta}=\left\{\alpha \in \mathcal{U}_{\mathbf{x}, \varrho}\right.$ : for some $\gamma<\lambda, b_{\bar{\alpha}_{\gamma}}=\bar{b}_{*}$ and $\left.\alpha=\alpha_{\gamma, \varrho}\right\}$.

For clause (e) recall $\bar{b}_{*} \in \mathcal{X}_{\bar{\alpha}, \varrho} \subseteq \mathcal{Z}_{\bar{\alpha}_{\gamma}, \varrho}$.

As $\varrho \in \operatorname{frt}(\mathbf{x})$, we are left with Case 2:

Case 2: $0=\left|\Lambda_{\mathbf{x}} \cap\left\{\varrho^{\wedge}\langle 0\rangle, \varrho^{\wedge}\langle 1\rangle\right\}\right|$ and let $\iota=0$. The only difference is that

- in clause $(\mathrm{a}), \operatorname{frt}\left(\Lambda_{\mathbf{y}}\right)=\Lambda \cup\left\{\varrho^{\wedge}\langle\iota\rangle\right\}$

so we have to add

$(f)$ if $\eta=\varrho$ then $\mathcal{U}_{\mathbf{y}, \eta}=\mathcal{W}_{\bar{\alpha}_{\gamma}}$ for some $\gamma<\lambda$ such that $\bar{b}_{\bar{\alpha}_{\gamma}}=\bar{b}_{*}$. 
Step 5: Finish. Recalling Step 2, we have shown that the assumption of $|A|^{+}$pairwise 1-contradictory higher formulas contradicts $\mathrm{NSOP}_{2}$. This completes the proof.

In this theorem, replacing 1-contradictory by $n$-contradictory would be straightforward as we can replace $\varphi$ by a conjunction, defined as follows.

Definition 8.22. Given $\varphi$ and $n$, let

$$
\begin{aligned}
\varphi_{[n]} & =\varphi_{[n]}\left(x, \bar{y}_{[n]}\right) \\
& =\varphi_{[n]}\left(\bar{x},\left\langle y_{i}: i<\ell g(\bar{y}) \cdot n\right\rangle\right) \\
& =\bigwedge_{\ell<n} \varphi\left(\bar{x},\left\langle y_{\ell g(\eta) \cdot \ell+j}: j<\ell g(\bar{y})\right\rangle\right) .
\end{aligned}
$$

We say $\varphi=\varphi(\bar{x}, \bar{y})$ has $\mathrm{NSOP}_{2}$ robustly when no $\varphi_{[n]}$ has $\mathrm{SOP}_{2}$.

Observation 8.23. If $T$ is $\mathrm{NSOP}_{2}$ then every $\varphi(\bar{x}, \bar{y}) \in \mathcal{L}\left(\tau_{T}\right)$ has $\mathrm{NSOP}_{2}$ robustly in $T$.

In the last main result of this section, we apply Theorem 6.14 characterizing the maximal class in $\unlhd^{*}$ to prove another property of $S O P_{2}$, related to so-called exact saturation (meaning for which singular $\kappa$ a given theory $T$ has a model which is $\kappa$-saturated but not $\kappa^{+}$-saturated). Exact saturation was studied in Shelah [22] $\S 2$ (pps. 31-37) and in a manuscript of Kaplan and Shelah [7] in preparation, which deal with elementary classes and $(\mathbf{D}, \kappa)$-sequence homogeneity.

Definition 8.24 ( see [22, [7] ). For any theory $T$, define

$$
\begin{aligned}
\operatorname{spec}(T)= & \{\kappa: \kappa \geq|T|, \kappa \text { singular } \\
& \text { and there exists a } \left.\kappa \text {-saturated not } \kappa^{+} \text {-saturated model of } T\right\} .
\end{aligned}
$$

The papers [22] and [7] find sufficient conditions but not necessary and sufficient conditions for exact saturation. In the present paper, we define a pseudo-elementary version 23 of this spectrum, which we will connect to $S O P_{2}$ via $\unlhd^{*}$.

Definition 8.25. The exact saturation spectrum for $T$ is defined to be the set:

$$
\begin{aligned}
\operatorname{ESS}(T)= & \left\{(\kappa, \mu): \kappa \geq \mu \geq|T|, \kappa \text { singular and for any } T_{1} \supseteq T,\left|T_{1}\right| \leq \mu,\right. \\
& \text { there is a } \left.\kappa \text {-saturated not } \kappa^{+} \text {-saturated member of } P C\left(T_{1}, T\right)\right\} .
\end{aligned}
$$

In future work, we hope to be able to get necessary and sufficient conditions for $\operatorname{ESS}(T)$ to be empty (at least restricting ourselves to $\kappa$ strong limit of large enough cofinality), and it seems plausible that this may be $S O P_{2}$. Here, using the methods of Section 6, we will prove one direction: if $T$ has $S O P_{2}$ then $\operatorname{ESS}(T)=\emptyset$, and discuss several open questions. First we recall two known examples.

\footnotetext{
${ }^{23}$ Compare the results of [16] VI.5 connecting the minimum class in Keisler's order to saturation properties of a $P C$-class.
} 
Fact 8.26 (22, 7]). Let $T$ be the theory of dense linear order without endpoints. Then for any singular $\kappa \geq|T|$, if $M \models T$ is $\kappa$ saturated then it is $\kappa^{+}$-saturated. Thus $\operatorname{spec}(T)=\emptyset$.

Proof. By quantifier elimination, it suffices to show that the cofinality and coinitiality of $M$ are at least $\kappa$ and for any regular $\kappa_{1}, \kappa_{2}$, every $\left(\kappa_{1}, \kappa_{2}\right)$-pre-cut is filled. Since cofinality and coinitiality of the model and of pre-cuts are necessarily regular cardinals, the result is immediate.

Fact 8.27 (22] Example 2.23). There is a theory $T$ with the independence property such that: if $T$ has an exactly $\kappa$-saturated model then $\kappa$ is regular. (In fact it is necessary and sufficient that $\kappa$ be regular.) Thus $\operatorname{spec}(T)=\emptyset$.

Recall the order $\triangleleft^{*}$ from Definition 6.7 above. In the following, countability is not essential.

Observation 8.28. Suppose $T_{0}, T_{1}$ are countable theories which are equivalent under the $\unlhd^{*}$ order. Then there exists a theory $T_{*}$ which interprets both $T_{0}$ and $T_{1}$, say via $\overline{\varphi_{0}}$ and $\overline{\varphi_{1}}$ respectively, such that: for any model $M \models T_{*}$, and any uncountable $\kappa$,

$$
N^{\left[\overline{\varphi_{0}}\right]} \text { is } \kappa \text {-saturated if and only if } N^{\left[\overline{\varphi_{1}}\right]} \text { is } \kappa \text {-saturated. }
$$

Proof. We may assume $T_{0}$ and $T_{1}$ have no finite models. Let $\mu$ be such that we have $T_{a}$ witnessing $T_{0} \unlhd^{*} T_{1}$ and $T_{b}$ witnessing $T_{1} \unlhd^{*} T_{0}$, and we may assume $\left|T_{a}\right|,\left|T_{b}\right|<\mu$. Let $\bar{\varphi}_{\ell}^{a}$ interpret $T_{\ell}$ in $T_{a}$ for $\ell=0,1$, and let $\bar{\varphi}_{\ell}^{b}$ interpret $T_{\ell}$ in $T_{b}$ for $\ell=0,1$. Without loss of generality:

- $\tau\left(T_{0}\right)$ and $\tau\left(T_{1}\right)$ are disjoint.

- $\bar{\varphi}_{\ell}^{a}$ says that for any model $M \models T_{a}$ the universe of $M^{\left[\bar{\varphi}_{\ell}^{a}\right]}$ is $\left(P_{\ell}^{a}\right)^{M}$, for some unary predicate $P_{\ell}^{a}$, and $T_{a}$ implies that $P_{0}^{a}$ and $P_{1}^{a}$ are disjoint;

- similarly for $T_{b}$.

- $\bar{\varphi}_{\ell}^{a}$ is the identity, where this means:

$-\tau\left(T_{a}\right) \supseteq\left(\tau\left(T_{0}\right) \cup \tau\left(T_{1}\right)\right)$

- $P \in \tau\left(T_{\ell}\right)$ implies $\varphi_{\ell, P}^{a}=P$, and $T_{a} \vdash(\forall \bar{x})\left(P(\bar{x}) \Longrightarrow \forall_{\ell<\lg (\bar{x})} P_{\ell, a}(x)\right)$

- similarly for function symbols $F \in \tau\left(T_{\ell}\right)$, adding that they are interpreted as partial functions with domain the predicate $P_{\ell}^{a}$ (alternately, we could have assumed without loss of generality that $\tau\left(T_{\ell}\right)$ has only predicates).

- each $\bar{\varphi}_{\ell}^{b}$ is likewise the identity.

- $\tau\left(T_{a}\right) \cap \tau\left(T_{b}\right)=\left(\tau\left(T_{0}\right) \cup \tau\left(T_{1}\right)\right)$.

What remains is to find models $M_{a} \models T_{a}$ and $M_{b} \models T_{b}$ such that the interpretation of $T_{\ell}$ in $M_{a}$ and $M_{b}$ are isomorphic. Given such models, we may finish by adding additional symbols giving a bijection between the respective interpretations of $T_{0}$ and $T_{1}$.

One way to construct such models is to recall that ultrapowers commute with reducts, and that any two elementarily equivalent models have isomorphic ultrapowers. Begin with $M_{0, a} \models T_{a}$ and $M_{0, b}=T_{b}$. Choose the ultrafilter $\mathcal{D}_{0}$ so that the 
$\mathcal{D}_{0}$-ultrapowers of $M_{0, a}^{\left[\bar{\varphi}_{0}^{a}\right]}$ and $M_{0, b}^{\left[\bar{\varphi}_{0}^{a}\right]}$ are isomorphic. Call these ultrapowers $M_{1, a}$ and $M_{1, b}$ respectively. (As ultrapowers commute with reducts, we may consider $M_{1, a}$ and $M_{1, b}$ as models of the full $T_{a}$ and $T_{b}$ respectively.) Consider the model $M_{c}$ which is the disjoint union of $M_{1, a}$ and $M_{1, b}$ and expand $M_{c}$ by adding symbols giving the isomorphism between $M_{1, a}^{\left[\bar{\varphi}_{0}^{a}\right]}$ and $M_{1, b}^{\left[\bar{\varphi}_{0}^{a}\right]}$. Let $M_{c}^{\left[\bar{\varphi}_{1}^{a}\right]}$ have the obvious meaning of $M_{1, a}^{\left[\bar{\varphi}_{1}^{a}\right]}$ considered within this model $M_{c}$. Next, choose the ultrafilter $\mathcal{D}_{1}$ so that $M_{c}^{\left[\bar{\varphi}_{1}^{a}\right]}$ and $M_{c}^{\left[\bar{\varphi}_{1}^{a}\right]}$ are isomorphic. Let $M_{*}$ be the $\mathcal{D}_{1}$-ultrapower of $M_{c}$. In this model we may expand $T_{c}$ so as to make the interpretations of $T_{1}$ isomorphic. Let $T_{*}$ be the theory of this expanded model. This completes our construction.

We had fixed an infinite $\mu$ so that $\left|T_{a}\right|+\left|T_{b}\right|<\mu$, and $\left|T_{0}\right|+\left|T_{1}\right|=\aleph_{0}$ by assumption. The construction gives that also $\left|T_{*}\right|<\mu$.

\section{Corollary 8.29.}

(1) Let $T_{0}$ be any theory for which $\operatorname{spec}\left(T_{0}\right)=\emptyset$. Let $T_{1}$ be any theory such that $T_{0}$ and $T_{1}$ are equivalent in the order $\unlhd^{*}$. Then $\operatorname{ESS}\left(T_{1}\right)=\emptyset$.

(2) Suppose $T_{0}, T_{1}$ are $\triangleleft^{*}$-equivalent, as witnessed by $T_{*}$ with $\left|T_{*}\right| \leq \mu$. Suppose $\kappa \geq \mu$ is singular and $(\kappa, \mu) \notin \operatorname{ESS}\left(T_{0}\right)$. Then $(\kappa, \mu) \notin \operatorname{ESS}\left(T_{1}\right)$.

Proof. (1) Let $T_{*}$ be a theory witnessing their equivalence, such as that given by Observation 8.28 . Let Let $\overline{\varphi_{0}}$ and $\overline{\varphi_{1}}$ witness the interpretations for $T_{0}$ and of $T_{1}$, respectively, and let $N^{[\bar{\varphi}]}$ and $N^{\left[\overline{\varphi_{*}}\right]}$ denote the respective interpretations in a given model $N \models T_{*}$. Now let $\kappa$ be a singular cardinal, $\kappa>\left|T_{1}\right|$ and suppose $M \in P C\left(T_{*}, T_{1}\right)$ is $\kappa$-saturated. Let $N \models T_{*}$ with $M=N \uparrow \tau\left(T_{1}\right)$ witness that $M \in P C\left(T_{*}, T_{1}\right)$. The fact that $M$ is $\kappa$-saturated says precisely that $N^{\left[\varphi_{1}\right]}$ is $\kappa$ saturated. By hypothesis of equivalence, $N^{\left[\overline{\varphi_{0}}\right]}$ is $\kappa$-saturated as well. By choice of $T_{0}, N^{\left[\overline{\varphi_{1}}\right]}$ is $\kappa^{+}$-saturated. Applying the hypothesis of equivalence in the other direction, $N^{\left[\overline{\varphi_{0}}\right]}$ is $\kappa^{+}$-saturated. This completes the proof.

(2) As $(\kappa, \mu) \notin \operatorname{ESS}\left(T_{0}\right)$ there is a theory $T_{00} \supseteq T_{0},\left|T_{00}\right| \leq \mu$, and a $\kappa$-saturated not $\kappa^{+}$-saturated member of $P C\left(T_{00}, T_{0}\right)$. In order to make use of this, we would first modify the construction of the theory $T_{*}$ (by means of a better choice of $T_{a}, T_{b}$ ) so that for any $M_{*} \models T_{*}$ we have that $M_{*}{ }^{\left[\overline{\varphi_{0}}\right]} \in P C\left(T_{00}, T_{0}\right)$. Since $\left|T_{00}\right| \leq \mu$, we can do this while preserving $\left|T_{*}\right| \leq \mu$. Then the proof continues as in part (1).

Theorem 8.30. If $T$ has $S O P_{2}$ then $\operatorname{ESS}(T)=\emptyset$. That is, if $T$ has $S O P_{2}$ then for some $T_{1} \supseteq T$ of cardinality $|T|$, for every singular $\kappa>|T|$, if $M \in P C\left(T_{1}, T\right)$ is $\kappa$-saturated then it is $\kappa^{+}$-saturated.

Proof. By Theorem 6.14, Fact 8.26 and Corollary 8.29(1).

Question 8.31. Does Theorem 8.30 hold for the theory $T_{r g}$ of the random graph?

In the case of $\operatorname{spec}\left(T_{r g}\right)$ rather than $\operatorname{ESS}\left(T_{r g}\right)$, note that:

Claim 8.32. If $\kappa>\operatorname{cf}(\kappa)+|T|$ then $\kappa \in \operatorname{spec}\left(T_{r g}\right)$.

Proof. Let $M$ be a $\kappa^{+}$-saturated model of $T_{r g}$. Let $\left\langle a_{\alpha}: \alpha<\kappa\right\rangle$ be pairwise distinct members of $M$ which form an empty graph, i.e. $\alpha<\beta<\lambda \Longrightarrow M \models \neg R\left(a_{\alpha}, a_{\beta}\right)$. 
Consider the submodel $N \subseteq M$ whose domain is

$$
\left\{b \in M:\left(\exists^{<\kappa} \alpha<\kappa\right)\left(R\left(b, a_{\alpha}\right)\right)\right\} .
$$

Then $N$ is a model of $T_{r g}$ which is as required: it is $\kappa$-saturated but $\left\{R\left(x, a_{\alpha}\right)\right.$ : $\alpha<\kappa\}$ is omitted so it is not $\kappa^{+}$-saturated.

Before giving some further evidence, we record here that there are other natural directions these investigations could take.

Discussion 8.33. Instead of asking whether $\kappa \in \operatorname{ESS}(T)$ for $\kappa>\operatorname{cf}(\kappa)+|T|$, we may fix a specific way to construct a $\kappa$-saturated model and ask if this implies that the constructed model is $\kappa^{+}$-saturated. For example:

(a) we may consider $T$ dependent, $|M|=\left\{a_{\alpha}: \alpha<\alpha_{*}\right\}, u_{\alpha} \in[\alpha]^{<\kappa}, \operatorname{tp}\left(a_{\alpha},\left\{a_{\beta}\right.\right.$ : $\beta<\alpha\})$ does not split over $\left\{a_{\beta}: \beta<\alpha\right\}$, and $M$ is $\kappa$-saturated; see [20].

(b) as in (a), but we may ask that $T$ has Skolem functions.

(c) as in (b), and also $\operatorname{tp}\left(a_{\alpha},\left\{a_{\beta}: \beta<\alpha\right\}\right)$ is finitely satisfiable in $\operatorname{Sk}\left(\left\{a_{\beta}\right.\right.$ : $\left.\left.\beta \in u_{\alpha}\right\}\right)$, see [16], VII, section 4.

(d) we may consider models built using $\mathcal{P}^{-}(n)$-diagrams in some explicit way.

Returning to ESS, on the positive side we can settle the case of stable $T$.

Claim 8.34. Let $T$ be a complete theory. If $T$ is stable, $\kappa=\kappa^{|T|}>\operatorname{cf}(\kappa)$, and $\kappa \geq \mu \geq T$, then $(\kappa, \mu) \in \operatorname{ESS}(T)$.

Proof. Recall that under these cardinal hypotheses there is a model of $T$ of cardinality $\kappa$ which is $\kappa$-saturated, see [16] Chapter III Theorem 3.12. Clearly this model is not $\kappa^{+}$-saturated. Given any $T_{1} \supseteq T,\left|T_{1}\right| \leq \mu$, we may build a model of $T_{1}$ of cardinality $\kappa$ in the same way whose restriction to $T$ is $\kappa$-saturated but evidently not $\kappa^{+}$-saturated.

Question 8.35. What about simple theories?

Problem 8.36. Determine whether the following is true: for $T$ complete and countable,

$$
\operatorname{ESS}(T)=\emptyset \text { if and only if } T \text { has } \mathrm{SOP}_{2} \text {. }
$$

These investigations suggest some interesting parallel questions for ultrafilters. The reader may recall that if $\lambda$ is a singular cardinal and the ultrafilter is $\lambda$-good then it is $\lambda^{+}$-good (since linear order is in the maximal Keisler class, [16].VI.2, a proof similar to that of Fact 8.26 goes through).

Problem 8.37. Suppose $\lambda$ is singular. Does there exist a regular ultrafilter which $\lambda$-saturates but does not $\lambda^{+}$-saturate ultrapowers of the random graph?

The property of an ultrafilter being " $\left(\lambda, \aleph_{0}\right)$-perfect" was defined in [10].

Problem 8.38. Suppose $\lambda$ is singular. If $\mathcal{D}$ is a regular ultrafilter which is $\left(\kappa, \aleph_{0}\right)$ perfect for every $\kappa<\lambda$, is $\mathcal{D}$ also $\left(\lambda^{+}, \aleph_{0}\right)$-perfect? If not, must it at least produce $\lambda^{+}$-saturated ultrapowers of the random graph? 


\section{REFERENCES}

[1] M. Džamonja and S. Shelah, "On $\triangleleft^{*}$-maximality," Annals of Pure and Applied Logic 125 (2004) 119-158. References are to the extended edition available at http://shelah.logic.at/listb.html, Paper 692.

[2] H. Gaifman, "Models and types of Peano arithmetic." Ann. Math. Logic. 9 (1976), no. 3, 223-306.

[3] H. Gaifman and C. Dimitracopoulos, "Fragments of Peano's arithmetic and the MRDP theorem." Logic and Algorithmic, Monographie No. 30 de L'Enseignement Mathématique, Genève,(1982) 187-206.

[4] H. J. Keisler, "Ultraproducts which are not saturated." J. Symbolic Logic 32 (1967) 23-46.

[5] R. Kossak, private communication.

[6] R. Kossak and J. Schmerl. The Structure of Models of Peano Arithmetic. Oxford University Press, 2006.

[7] I. Kaplan and S. Shelah, manuscript F1473, in preparation.

[8] M. Malliaris and S. Shelah, "Cofinality spectrum theorems for model theory, set theory and general topology." Accepted, J. AMS. References are to the revised version.

[9] M. Malliaris and S. Shelah, "General topology meets model theory, on $\mathfrak{p}$ and t." PNAS 2013 110 (33) 13300-13305.

[10] M. Malliaris and S. Shelah, "Keisler's order has infinitely many classes." Manuscript 1050, in preparation.

[11] J. Moore, "Model theory and the cardinals $\mathfrak{p}$ and t." PNAS August 13, 2013 vol. 110 no. 33 $13238-13239$.

[12] R. MacDowell and E. Specker, "Modelle der arithmetic." Infinitistic Methods, Proceedings of the Symposium on Foundations of Mathematics, Warsaw, 1959. Pergamon Press, Oxford, 1961 pp. 257-263.

[13] J.-F. Pabion. "Saturated models of Peano arithmetic." J. Symbolic Logic 47 (1982), 625-637.

[14] A. J. Wilkie and J. B. Paris, "On the scheme of induction for bounded arithmetic formulas." Annals of Pure and Applied Logic 35 (1987) 261-302.

[15] J. Schmerl, "Elementary cuts in saturated models of Peano arithmetic." manuscript.

[16] S. Shelah, Classification Theory, North-Holland, 1978. Rev. ed. 1990.

[17] S. Shelah, "End extensions and numbers of countable models." J Symbolic Logic 43 (1978) 550-562

[18] S. Shelah, "Toward classifying unstable theories." Annals of Pure and Applied Logic 80 (1996) $229-255$.

[19] S. Shelah, "General non-structure theory." Paper E59, available at http://shelah.logic.at/

[20] S. Shelah, "Classification theory for elementary classes with the dependence property - a modest beginning". Scientiae Math Japonicae 59, No. 2; (special issue: e9, 503-544) (2004) $265-316$

[21] S. Shelah, "Quite complete real closed fields." Israel J Math 142 (2004) 261-272. Extended version available at http://shelah.logic.at/files/757.pdf

[22] S. Shelah, paper 900.

[23] S. Shelah, "Models of expansions of $\mathbb{N}$ with no end extensions." Math Logic Quarterly 57 (2011) 341-365

[24] S. Shelah and A. Usvyatsov, "More on $\mathrm{SOP}_{1}$ and $\mathrm{SOP}_{2}$." Ann. Pure Appl. Logic 155 (2008) $16-31$. 
Department of Mathematics, University of Chicago, 5734 S. University Avenue, Chicago, IL 60637, USA and Mathematical Sciences Research Institute, 17 Gauss Way, Berkeley, CA 94720, USA

E-mail address: mem@math.uchicago.edu

Einstein Institute of Mathematics, Edmond J. Safra Campus, Givat Ram, The Hebrew University of Jerusalem, Jerusalem, 91904, Israel, and Department of Mathematics, Hill Center - Busch Campus, Rutgers, The State University of New Jersey, 110 Frelinghuysen Road, Piscataway, NJ 08854-8019 USA

E-mail address: shelah@math.huji.ac.il

URL: http://shelah.logic.at 